\title{
BIOMETRIKA
}

\section{ON THE STANDARD DEVIATIONS OF ADJUSTED AND INTERPOLATED VALUES OF AN OBSERVED POLY- NOMIAL FUNCTION AND ITS CONSTANTS AND THE GUIDANCE THEY GIVE TOWARDS A PROPER CHOICE OF THE DISTRIBUTION OF OBSERVATIONS.}

\author{
By KIRSTINE SMITH, Copenhagen.
}

\begin{tabular}{|c|c|c|}
\hline & TENTS & GGE \\
\hline I. & $\begin{array}{l}\text { Introduction } \cdot \text { Adjustment of a polynomial function of one variable; general } \\
\text { distribution of observations } .\end{array}$ & 3 \\
\hline II. & The "best" grouping of observations with constant standard deviation & 13 \\
\hline III. & $\begin{array}{l}\text { Uniform continuous distribution of observations with constant } \\
\text { standard deviation. General formulae . . . . . }\end{array}$ & 17 \\
\hline IV. & $\begin{array}{l}\text { Uniform continuous distribution of observations with constant } \\
\text { standard deviation. Special formulae. . . . }\end{array}$ & 28 \\
\hline V. & $\begin{array}{l}\text { Uniform continuous distribution of observations with additional } \\
\text { observations clustered at the ends of the range; constant } \\
\text { standard deviation of observations. General formulae. }\end{array}$ & 31 \\
\hline VI. & $\begin{array}{l}\text { Uniform continuous distribution of observations with additional } \\
\text { clusters at the ends of the range; constant standard deviation }\end{array}$ & \\
\hline & of observations. Special formulae. & 41 \\
\hline VII. & Observations with varying standard deviation & 50 \\
\hline $\mathrm{NTT}$ & $\begin{array}{l}\text { Best distribution of observations for determining a single constant } \\
\text { of the function }\end{array}$ & 72 \\
\hline IX. & $\begin{array}{l}\text { Adjustment with regard to both of two variates connected by a } \\
\text { linear relation . } .6 \cdot c \cdot c \cdot c c c c\end{array}$ & 82 \\
\hline & & \\
\hline
\end{tabular}

In all sorts of experiments which are not simple repetitions but have at least one varying essential circumstance or indefinite variate the experimentalist is confronted with a choice in regard to the values of that variate. If the experiments be quite simple the question may be without great importance; but when their requirements as to time or expenditure come into account the problem arises, how the observations should be chosen in order that a limited number of them may give the maximum amount of knowledge. It clearly depends upon the relationship between the observed quantity, which we shall name the primary variate, and its essential circumstances, the secondary variates, and upon the variation of the errors of the observations. 


\section{Choice in the Distribution of Observations}

When we deal with, for example, a linear function which it is possible to observe with the same accuracy for all values of the indefinite variate we should not hesitate to put the observations in two equally big groups as far apart from each other as feasible. But if the standard deviation of the observations be a function of the indefinite variate and increases with the distance from the middle of the range, where is then the point in which the advantage of removing the two groups of observations from each other just counterbalances the disadvantages of increasing the error of observations? The problem becomes very complicated for functions of higher degrees.

We shall in this memoir try to contribute to the solution in the case of polynomial functions by examining the standard deviations of the adjusted and more especially the interpolated values of such functions for different distributions of observations. Those values inside the working range of observations may be considered the sum of knowledge acquired by the experiments. The adjusted values outside the working range may probably in exceptional cases be of interest, but as only by some other type of experiment we can make sure that the form of function holds outside the range they are in ordinary cases without great value. We shall therefore aim at finding the distribution of observations which within the selected range gives the most satisfactory standard deviations of the adjusted values of the function.

To consider the standard deviations satisfactory we must of course demand that they shall be as small as possible, and since a greater accuracy in one part may be expected to be accompanied by a smaller accuracy in another part we want them in addition to be as near constant as possible. In other words the curve of standard deviation with the lowest possible maximum value within the working range of observations is what we shall attempt to find. It appears that the distribution of observations which fulfils this demand consists of specially placed groups in number just sufficient to determine the constants of the function. We shall accordingly pay attention also to the desirability usually present of ascertaining the form of function by means of the observations. As might be expected we find that the standard deviations obtained from a uniform continuous distribution of observations increase towards the ends of the range. By choosing a uniform continuous distribution with additional clusters at the ends of the range we shall try to find a compromise between the two desiderata of a low maximum of standard deviation and of a uniform distribution.

The indefinite variate is supposed to have a vanishing error of observation compared with that of the principal variate. This error may be constant or varying with the indefinite variate, but in either case it is supposed to follow the typical law so closely that the method of least squares may satisfactorily be applied to the observations. After having found first the most advantageous distributions for observations of functions up to the sixth degree with constant standard deviations we examine the case for observations of functions of the first and of the second degree which have standard deviations of the form $\sigma(1+\alpha x)$ and $\sigma\left(1+\alpha x^{2}\right)$. If it is profitable to use the whole of the working range the latter distributions 


\section{Kirstine Smith}

are practically found from the former by multiplying their frequencies by the squared standard deviations of the observations at the corresponding place. But in cases where extrapolation is of advantage, and the whole range therefore not to be used, the law of the frequencies has to be examined anew.

In Section VIII we find for the same two cases of varying error of observation the distributions which make each single constant of a function of the first and of the second degree a minimum.

\section{Adjustment of a polynomial function of one variable; general distribution} of observations.

(1) Let $y_{1}, y_{2} \ldots \ldots y_{p} \ldots \ldots y_{N}$ be $N$ observations of a function of $n$th degree taken at the points $x_{1}, x_{2} \ldots \ldots x_{p} \ldots \ldots x_{N}$,

$$
y=a_{0}+a_{1} x+a_{2} x^{2}+\ldots \ldots+a_{n} x^{n}
$$

Let us assume that from earlier experience we know the standard deviation of an observation of $y$ to be $\sigma \sqrt{f(x)}$. The method of least squares will then give us the following system of normal equations in which the sums are to be extended over all the observations:

$$
\begin{aligned}
& S\left\{\frac{y_{p}}{f\left(x_{p}\right)}\right\}=S\left\{\frac{1}{f\left(x_{p}\right)}\right\} a_{0}+S\left\{\frac{x_{p}}{f\left(x_{p}\right)}\right\} a_{1}+S\left\{\begin{array}{c}
x_{p}^{2} \\
f\left(x_{p}\right)
\end{array}\right\} a_{2}+\ldots \ldots+S\left\{\begin{array}{c}
x_{p}^{n} \\
f\left(x_{p}\right)
\end{array}\right\} a_{n} \\
& S\left\{\frac{y_{p} x_{p}}{f\left(x_{p}\right)}\right\}=S\left\{\frac{x_{p}}{f\left(x_{p}\right)}\right\} a_{0}+S\left\{\frac{x_{p}^{2}}{f\left(x_{p}\right)}\right\} a_{1}+S\left\{\frac{x_{p}^{3}}{f\left(x_{p}\right)}\right\} a_{2}+\ldots \ldots+S\left\{\frac{x_{p}^{n+1}}{f\left(x_{p}\right)}\right\} a_{n} \\
& \left.S\left\{\begin{array}{c}
y_{p} x_{p}^{2} \\
f\left(x_{p}\right)
\end{array}\right\}=S\left\{\frac{x_{p}^{2}}{f\left(x_{p}\right)}\right\} a_{0}+S\left\{\begin{array}{c}
x_{p}^{3} \\
f\left(x_{p}\right)
\end{array}\right\} a_{1}+S\left\{\begin{array}{c}
x_{p}^{4} \\
f\left(x_{p}\right)
\end{array}\right\} a_{2}+\ldots \ldots+S\left\{\begin{array}{c}
x_{p}^{n+2} \\
f\left(x_{p}\right)
\end{array}\right\} a_{n}\right\} \\
& \left.\left.\begin{array}{cccc}
\vdots & \vdots & \vdots & \vdots \\
& S\left\{y_{p} x_{p}^{n}\right. \\
f\left(x_{p}\right)
\end{array}\right\}=S\left\{\frac{x_{p}^{n}}{f\left(x_{p}\right)}\right\} a_{0}+S\left\{\frac{x_{p}^{n+1}}{f\left(x_{p}\right)}\right\} a_{1}+S\left\{\frac{x_{p}^{n+2}}{f\left(x_{p}\right)}\right\} a_{2}+\ldots \ldots+S\left\{\begin{array}{c}
x_{p}^{2 n} \\
f\left(x_{p}\right)
\end{array}\right\} a_{n}\right\}
\end{aligned}
$$

If $f(x)$ is 1 the sums are the moment coefficients of the places of observations multiplied by $N$, and in the general case we shall for brevity put

$$
S\left\{\frac{x_{p}^{r}}{f\left(x_{p}\right)}\right\}=N \cdot m_{r} .
$$

By elimination of the $a$ 's between (1) and (2) we find

$$
\begin{array}{cccccc}
N . y & 1 & x & x^{2} & \ldots \ldots & x^{n} \\
S\left\{\frac{y_{p}}{f\left(x_{p}\right)}\right\} & m_{0} & m_{1} & m_{2} & \ldots \ldots & m_{n} \\
S\left\{\frac{y_{p} x_{p}}{f\left(x_{p}\right)}\right\} & m_{1} & m_{2} & m_{3} & \ldots \ldots & m_{n+1} \\
S\left\{\frac{y_{p} x_{p}^{2}}{f\left(x_{p}\right)}\right\} & m_{2} & m_{3} & m_{4} & \ldots \ldots & m_{n+2} \\
\vdots & \vdots & \vdots & \vdots & & \vdots \\
S\left\{\frac{y_{p} x_{p}^{n}}{f\left(x_{p}\right)}\right\} & m_{n} & m_{n+1} & m_{n+2} & \ldots \ldots & m_{2 n}
\end{array} \mid=0 \ldots \ldots \ldots \ldots(3),
$$

which determines the adjusted $y$ corresponding to the variable $x$. 
(2) To find the standard deviation $\sigma_{y_{r}}$ of an adjusted $y_{r}$ it will be easiest to start from the equations (2). If the first be multiplied by $a_{0}$, the second by $\alpha_{1}$ and so on before summing, and if we choose $\alpha_{0}, \alpha_{1} \ldots \ldots \alpha_{n}$ so that

$$
\begin{aligned}
& a_{0} m_{0}+a_{1} m_{1}+a_{2} m_{2}+\ldots \ldots+a_{n} m_{n}=1 \\
& a_{0} m_{1}+a_{1} m_{2}+a_{2} m_{3}+\ldots \ldots+a_{n} m_{n+1}=x_{r} \\
& \left.a_{0} m_{2}+\alpha_{1} m_{3}+a_{2} m_{4}+\ldots \ldots+\alpha_{n} m_{n+2}=x^{2}\right\}
\end{aligned}
$$

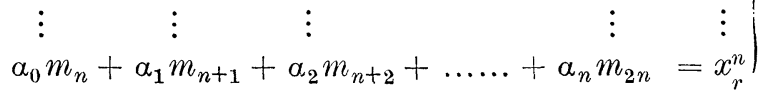

we find that

$$
y_{r}=\frac{1}{N} S\left\{\frac{y_{p}}{f\left(x_{p}\right)}\left[a_{0}+a_{1} x_{p}+a_{2} x_{p}^{2}+\ldots \ldots+a_{n} x_{p}^{n}\right]\right\},
$$

and therefore $\left.\sigma_{y_{r}}^{2}=\frac{\sigma^{2}}{N^{2}} S\left\{\frac{1}{f\left(x_{p}\right)}\left[\alpha_{0}+a_{1} x_{p}+a_{2} x_{p}^{2}+\ldots \ldots+\alpha_{n} x_{p}^{n}\right]\right]^{2}\right\}$.

By multiplying out the square this may be written

$$
\begin{aligned}
& \sigma_{y_{r}}^{2}=\frac{\sigma^{2}}{N}\left\{\alpha_{0}\left[\alpha_{0} m_{0}+\alpha_{1} m_{1}+\alpha_{2} m_{2}+\ldots \ldots+\alpha_{n} m_{n}\right]\right. \\
& +\alpha_{1}\left[\alpha_{0} m_{1}+\alpha_{1} m_{2}+\alpha_{2} m_{3}+\ldots \ldots+\alpha_{n} m_{n+1}\right] \\
& +\alpha_{2}\left[\alpha_{0} m_{2}+\alpha_{1} m_{3}+\alpha_{2} m_{4}+\ldots \ldots+\alpha_{n} m_{n+2}\right] \\
& \begin{array}{lllll}
\vdots & \vdots & \vdots & \vdots & \vdots
\end{array} \\
& \left.+\alpha_{n}\left[\alpha_{0} m_{n}+\alpha_{1} m_{n+1}+\alpha_{2} m_{n+2}+\ldots \ldots+\alpha_{n} m_{2 n}\right]\right\} \text {, }
\end{aligned}
$$

or applying (4)

$$
\sigma_{y_{r}}^{2}=\frac{\sigma^{2}}{N}\left(a_{0}+a_{1} x_{r}+\alpha_{2} x_{r}^{2}+\ldots \ldots+\alpha_{n} x_{r}^{n}\right) .
$$

Hence $\sigma_{y r}^{2}$ is found by elimination of the $a$ 's between (4) and (5), which results in

$$
\left|\begin{array}{cccccc}
\sigma_{y_{r}}^{2} \cdot \frac{N}{\sigma^{2}} & 1 & x_{r} & x_{r}^{2} & \ldots \ldots & x_{r}^{n} \\
1 & m_{0} & m_{1} & m_{2} & \ldots \ldots & m_{n} \\
x_{r} & m_{1} & m_{2} & m_{3} & \ldots \ldots & m_{n+1} \\
x_{r}^{2} & m_{2} & m_{3} & m_{4} & \ldots \ldots & m_{n+2} \\
\vdots & \vdots & \vdots & \vdots & & \vdots \\
x_{r}^{n} & m_{n} & m_{n+1} & m_{n+2} & \ldots \ldots & m_{2 n}
\end{array}\right|=0 \ldots
$$

This determinant is of fundamental importance for all the following work and it will be useful at once to examine it more closely.

(3) First however it may be pointed out that the standard deviation of any other linear function $\quad \phi=b_{0} a_{0}+b_{1} a_{1}+b_{2} a_{2}+\ldots \ldots b_{n} a_{n}$ of the constants of the function $y$ may be determined in quite the same way by

$$
\left|\begin{array}{cccccc}
\sigma_{\phi}^{2} \cdot \frac{N}{\sigma^{2}} & b_{0} & b_{1} & b_{2} & \ldots \ldots & b_{n} \\
b_{0} & m_{0} & m_{1} & m_{2} & \ldots \ldots & m_{n} \\
b_{1} & m_{1} & m_{2} & m_{3} & \ldots \ldots & m_{n+1} \\
b_{2} & m_{2} & m_{3} & m_{4} & \ldots \ldots & m_{n+2} \\
\vdots & \vdots & \vdots & \vdots & & \vdots \\
b_{n} & m_{n} & m_{n+1} & m_{n+2} & \ldots \ldots & m_{2 n}
\end{array}\right|=0 \ldots
$$


Kirstine Smith

In particular $\sigma_{a_{\boldsymbol{p}}}^{2}$ is found from

$$
\left|\begin{array}{cccccccc}
\sigma_{a_{p}}^{2} \cdot \frac{N}{\sigma^{2}} & 0 & 0 & 0 & \ldots & 1 & \ldots & 0 \\
0 & m_{0} & m_{1} & m_{2} & \ldots & m_{p} & \ldots & m_{n} \\
0 & m_{1} & m_{2} & m_{3} & \ldots & m_{p+1} & \ldots & m_{n+1} \\
0 & m_{2} & m_{3} & m_{A} & \ldots & m_{p+2} & \ldots & m_{n+2} \\
\vdots & \vdots & \vdots & \vdots & & \vdots & & \vdots \\
1 & m_{p} & m_{p+1} & m_{p+2} & \ldots & m_{2 p} & \ldots & m_{n+p} \\
\vdots & \vdots & \vdots & \vdots & & \vdots & & \vdots \\
0 & m_{n} & m_{n+1} & m_{n+2} & \ldots & m_{p+n} & \ldots & m_{2 n}
\end{array}\right|=0
$$

(4) Let us call a determinant, identical with that of (6) except that it has 0 instead of the element $\sigma_{y_{r}}^{2} \cdot \frac{N}{\sigma^{2}}$, $\Delta$, let $\Delta_{r, s}$ be its minor not containing the $r$ th row and $s$ th column, again let $\Delta_{r, \boldsymbol{s}, \mathfrak{p}, q}$ be the minor of this not containing the $p$ th row and the $q$ th column of $\Delta$. We then find from (8)

$$
\sigma_{a_{p}}^{2}=\frac{\sigma^{2}}{N} \frac{\Delta_{p+2, p+2,1,1}}{\Delta_{1,1}}
$$

With this notation we obtain from $(6)$

$$
\sigma_{y_{r}}^{2}=\frac{\sigma^{2}}{N}\left(-\frac{\Delta}{\Delta_{1,1}}\right)
$$

In the following we shall drop the index $r$ and indicate by ${ }_{n} \sigma_{y}$ the standard deviation of a $y$ adjusted by means of a function of the $n$th degree.

If we were dealing with a function of $(n-1)$ st degree and retained the observations distributed as before we should find

and therefore

$$
{ }_{n-1} \sigma_{y}^{2}=\frac{\sigma^{2}}{N}\left(-\frac{\Delta_{n+2, n+8}}{\Delta_{n+2, n+2,1,1}}\right),
$$

$$
{ }_{n} \sigma_{y}^{2}-{ }_{n-1} \sigma_{y}^{2}=\frac{\sigma^{2}}{N} \cdot \frac{\Delta_{1,1} \cdot \Delta_{n+2, n+2}-\Delta \cdot \Delta_{n+2, n+2,1,1}}{\Delta_{1,1} \cdot \Delta_{n+2, n+2,1,1}},
$$

but $\Delta$ is orthosymmetrical and therefore the numerator of this fraction equals $\Delta_{n+2,1}^{2}$, and

$$
{ }_{n} \sigma_{y}^{2}-{ }_{n-1} \sigma_{y}^{2}=\frac{\sigma^{2}}{N} \cdot \Delta_{1,1}^{2} \cdot \Delta_{n+2, n+2,1,1} .
$$

It was shown before that

$$
\sigma_{a_{n}}^{2}=\frac{\sigma^{2}}{\bar{N}} \cdot \frac{\Delta_{n+2, n+2,1,1}}{\Delta_{1,1}},
$$

hence $\Delta_{1,1}$ and $\Delta_{n+2, n+2,1,1}$ have the same sign, and ${ }_{n} \sigma_{y}^{2}-{ }_{n-1} \sigma_{y}^{2}$ is therefore a square of a function of $x$. In the same way we can express ${ }_{n-1} \sigma_{y y}^{2}-{ }_{n-2} \sigma_{y}^{2}$ and thus further down all the differences till $\sigma_{0} \sigma_{y}^{2}=\frac{\sigma^{2}}{N} \cdot \frac{1}{m_{0}}$ by which means ${ }_{n} \sigma_{y}^{2}$ is developed in a sum of squares and takes the shape 


$$
\begin{aligned}
& { }_{n} \sigma_{y}^{2}=\frac{\sigma^{2}}{N}\left\{\frac{1}{m_{0}}+\frac{\left|\begin{array}{cc}
1 & m_{0} \\
x & m_{1}
\end{array}\right|^{2}}{m_{0} \cdot\left|\begin{array}{cc}
m_{0} & m_{1} \\
m_{1} & m_{2}
\end{array}\right|}+\frac{\left|\begin{array}{ccc}
1 & m_{0} & m_{1} \\
x & m_{1} & m_{2} \\
x^{2} & m_{2} & m_{3}
\end{array}\right|^{2}}{\left|\begin{array}{cc}
m_{0} & m_{1} \\
m_{1} & m_{2}
\end{array}\right| \cdot\left|\begin{array}{ccc}
m_{0} & m_{1} & m_{2} \\
m_{1} & m_{2} & m_{3} \\
m_{2} & m_{3} & m_{4}
\end{array}\right|}+\ldots \ldots\right.
\end{aligned}
$$

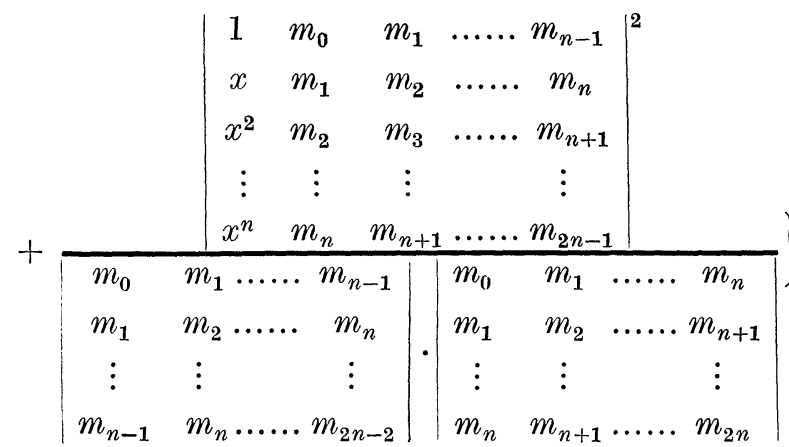

It will be seen that the squared standard deviation of an adjusted $y$ is a function of the $2 n$th degree of $x$. The coefficient of $x^{2 n}$ is the square of $\frac{\Delta_{n+2, n+2,1,1}}{\Delta_{1,1}}$ which, as was just seen, is the factor with which $\frac{\sigma^{2}}{N}$ should be multiplied in order to give $\sigma_{a_{n}}^{2}$, it is therefore positive and can never vanish.

(5) If all the $m$ 's with odd indices are zero it is seen from (6) that $\sigma_{y}^{2}$ is a function of $x^{2}$. This is, at least in theory, a natural thing to aim at, since our general purpose is to find a curve for $\sigma_{y}^{2}$ giving as nearly as possible a constant value for $\sigma_{y}^{2}$ throughout the range.

Rearranging the order of rows and columns in (6) we get, when all $m_{2 q+1}=0$ and $n=2 p$,

$$
\begin{array}{ccccccccccc}
{ }_{2 p} \sigma_{y}^{2} \cdot \frac{N}{\sigma^{2}} & 1 & x^{2} & x^{4} & \ldots \ldots & x^{2 p} & x & x^{3} & x^{5} & \ldots \ldots & x^{2 p-1} \\
1 & m_{0} & m_{2} & m_{4} & \ldots \ldots & m_{2 p} & 0 & 0 & 0 & \ldots \ldots & 0 \\
x^{2} & m_{2} & m_{4} & m_{6} & \ldots \ldots & m_{2 p+2} & 0 & 0 & 0 & \ldots \ldots & 0 \\
x^{4} & m_{4} & m_{6} & m_{8} & \ldots \ldots & m_{2 p+4} & 0 & 0 & 0 & \ldots \ldots & 0 \\
\vdots & \vdots & \vdots & \vdots & & \vdots & \vdots & \vdots & \vdots & & \vdots \\
x^{2 p} & m_{2 p} & m_{2 p+2} & m_{2 p+4} & \ldots \ldots & m_{4 p} & 0 & 0 & 0 & \ldots \ldots & 0 \\
x & 0 & 0 & 0 & \ldots \ldots & 0 & m_{2} & m_{4} & m_{6} & \ldots \ldots & m_{2 p} \\
x^{3} & 0 & 0 & 0 & \ldots \ldots & 0 & m_{4} & m_{6} & m_{8} & \ldots \ldots & m_{2 p+2} \\
x^{5} & 0 & 0 & 0 & \ldots \ldots & 0 & m_{6} & m_{8} & m_{10} & \ldots \ldots & m_{2 p+4} \\
\vdots & \vdots & \vdots & \vdots & & \vdots & \vdots & \vdots & \vdots & & \vdots \\
x^{2 p-1} & 0 & 0 & 0 & \ldots \ldots & 0 & m_{2 p} & m_{2 p+2} & m_{2 p+4} & \ldots \ldots & m_{4 p-2}
\end{array} \mid=0
$$




\section{KiRstine SMith}

from which we find

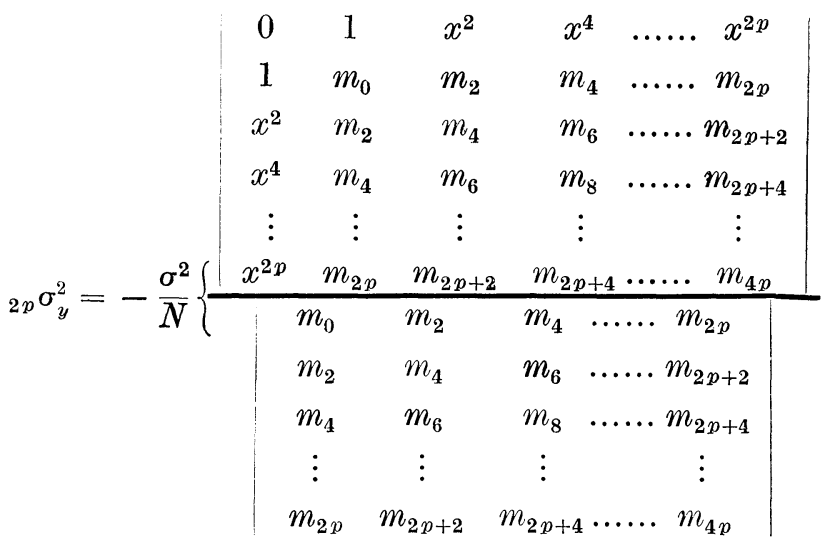

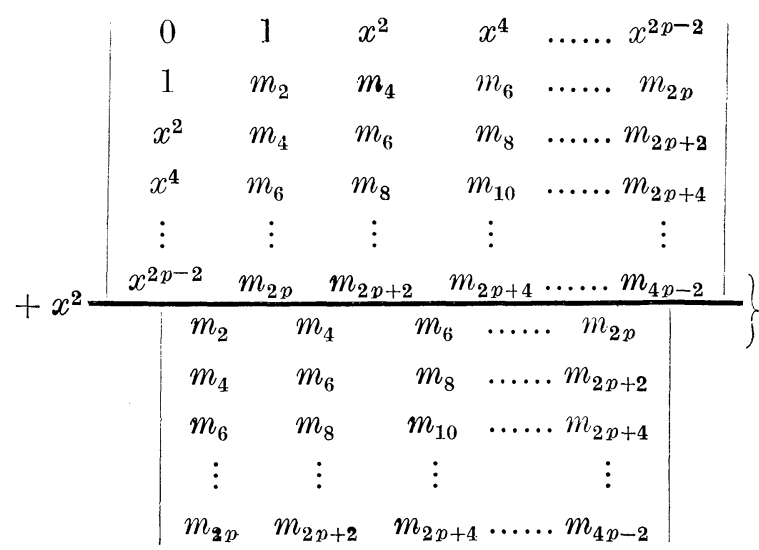

For a function of the degree $2 p-1$ we get the same determinant as in (12) except that it does not contain the row and column in which $x^{2 p}$ is found. Hence we find

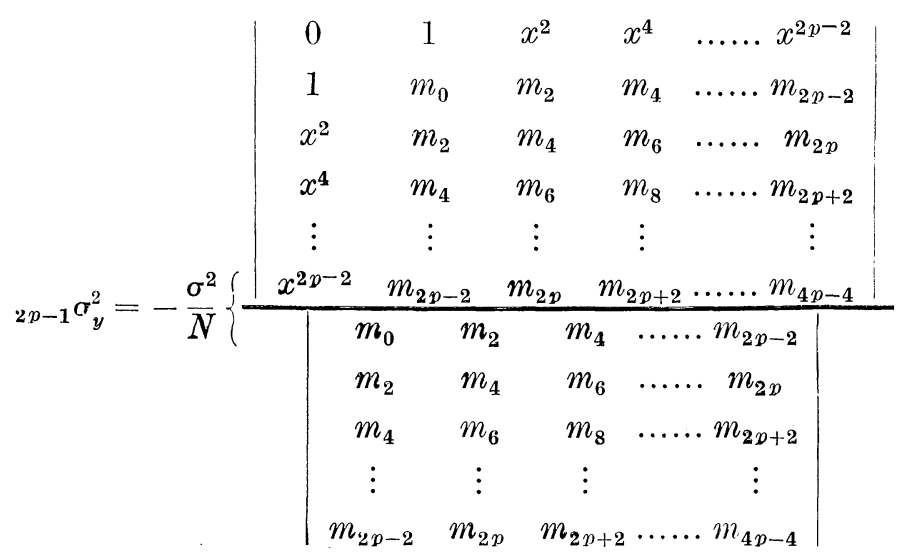




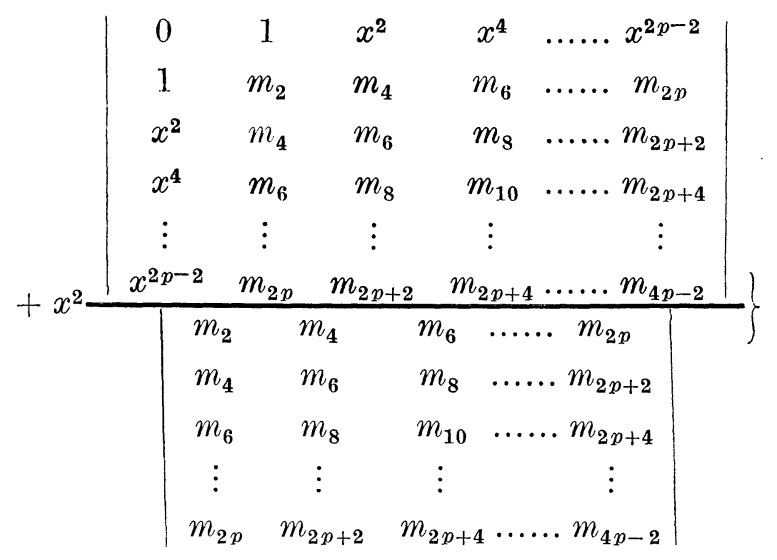

(6) The last two determinant ratios of (13) and (14) are identical, and when the numerator of the first fraction of (13) is indicated by $\delta$ we therefore find

or as $\delta$ is orthosymmetrical and therefore

$$
{ }_{2 p} \sigma_{y}^{2}-{ }_{2 p-1} \sigma_{y}^{2}=\frac{\sigma^{2}}{N}\left(\frac{\delta_{p+2, p+2}}{\delta_{1,1, p+2, p+2}}-\frac{\delta}{\delta_{1,1}}\right),
$$

$$
\begin{gathered}
\delta_{1,1} \cdot \delta_{p+2, p+2}-\delta . \delta_{1,1, p+2, p+2}=\delta_{p+2,1}^{2} \\
{ }_{2 p} \sigma_{y}^{2}-{ }_{2 p-1} \sigma_{y}^{2}=\frac{\sigma^{2}}{N} \cdot \frac{\delta_{p+2,1}^{2}}{\delta_{1,1} \delta_{p+2, p+2,1,1}} .
\end{gathered}
$$

Comparing ${ }_{2 p-2} \sigma_{y}^{2}$ and ${ }_{2 p-1} \sigma_{y}^{2}$ we see that they have the first determinant ratio in common and that when $\gamma$ stands for the numerator of the other fraction of $2 p-1 \sigma_{y}^{2}$ we have

$$
{ }_{2 p-1} \sigma_{y}^{2}-{ }_{2 p-2} \sigma_{y}^{2}=\frac{\sigma^{2}}{N} x^{2}\left(\frac{\gamma_{p+1, p+1}}{\gamma_{p+1, p+1,1,1}}-\frac{\gamma}{\gamma_{1,1}}\right),
$$

or again, since $\gamma$ is orthosymmetrical,

$$
{ }_{2 p-1} \sigma_{y}^{2}-{ }_{2 p-2} \sigma_{y}^{2}=\frac{\sigma^{2}}{N} x^{2} \frac{\gamma_{p+1,1}^{2}}{\gamma_{1,1} \cdot \gamma_{p+1, p+1,1,1}} .
$$

The general formula (11) hence for any $m_{2 q+1}=0$ takes the shape

$$
\begin{aligned}
& { }_{\mathbf{2} p} \sigma_{y}^{2}=\frac{\sigma^{2}}{N}\left\{\frac{1}{m_{0}}+x^{2} \frac{1}{m_{2}}+\frac{\left|\begin{array}{cc}
1 & m_{0} \\
x^{2} & m_{2}
\end{array}\right|^{2}}{m_{0} \cdot\left|\begin{array}{cc}
m_{0} & m_{2} \\
m_{2} & m_{4}
\end{array}\right|}+x^{2} \frac{\left|\begin{array}{cc}
1 & m_{2} \\
x^{2} & m_{4}
\end{array}\right|^{2}}{m_{2} \cdot \begin{array}{ll}
m_{2} & m_{4} \\
m_{4} & m_{6}
\end{array} \mid}+\ldots .\right.
\end{aligned}
$$

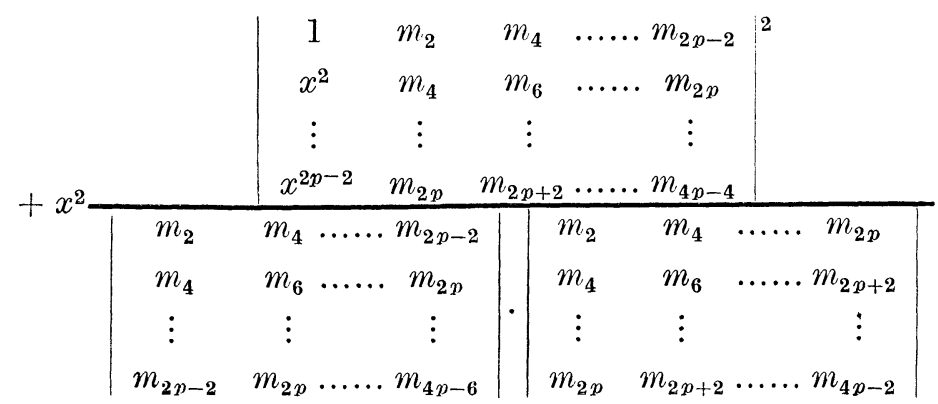




\section{KiRstine SMith}

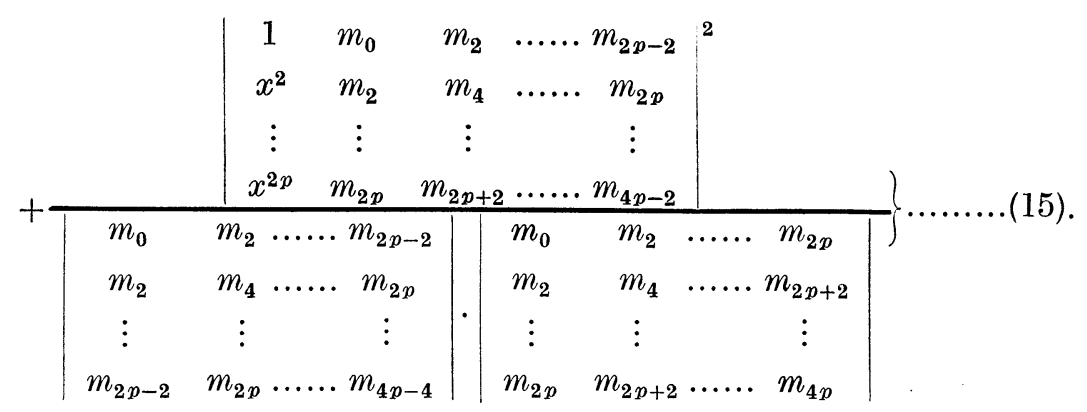

(7) Before leaving the general case and treating special distributions of observations three auxiliary propositions shall be proved. We shall first prove that the curve of ${ }_{n} \sigma_{y}^{2}$ can never be entirely below $\frac{\sigma^{2}}{N} \cdot \frac{n+1}{m_{0}}$. With that purpose ${ }_{n} \sigma_{y}^{2}$ will be summed over all the places of observation with the weight $\frac{1}{f(x)}$, i.e. for a continuous distribution of observations, the expression $\frac{\psi(x)}{f(x)}{ }_{n} \sigma_{y}^{2} d x$, where $\psi(x)$ is the number of observations, will be integrated over the range of observations.

Looking first at the numerator of the last term of (11) we find that it can be expanded into

$$
\begin{aligned}
& (-1)^{n+1}\left\{\begin{array}{ccccc}
1 & m_{0} & m_{1} & \ldots \ldots & m_{n-1} \\
x & m_{1} & m_{2} & \ldots \ldots & m_{n} \\
x^{2} & m_{2} & m_{3} & \ldots \ldots & m_{n+1} \\
\vdots & \vdots & \vdots & & \vdots \\
x^{n} & m_{n} & m_{n+1} & \ldots \ldots & m_{2 n-1}
\end{array}\left|\times \Delta_{1, n+2,2,1}+\right| \begin{array}{ccccc}
x & m_{0} & m_{1} & \ldots \ldots & m_{n-1} \\
x^{2} & m_{1} & m_{2} & \ldots \ldots & m_{n} \\
x^{3} & m_{2} & m_{3} & \ldots \ldots & m_{n+1} \\
\vdots & \vdots & \vdots & \\
x^{n+1} & m_{n} & m_{n+1} & \ldots . . & m_{2 n-1}
\end{array} \mid\right. \\
& \left.\times \Delta_{1, n+2,3,1}+\ldots \ldots+\left|\begin{array}{ccccc}
x^{n} & m_{0} & m_{1} & \cdots \cdots & m_{n-1} \\
x^{n+1} & m_{1} & m_{2} & \ldots \ldots & m_{n} \\
x^{n+2} & m_{2} & m_{3} & \ldots \ldots & m_{n+1} \\
\vdots & \vdots & \vdots & & \vdots \\
x^{2 n} & m_{n} & m_{n+1} & \ldots \ldots & m_{2 n-1}
\end{array}\right| \times \Delta_{1, n+2, n+2,1}\right\} .
\end{aligned}
$$

Now $\int \frac{\psi(x) x^{r}}{f(x)} d x$ integrated over all the observations is what we have called $N . m_{r}$. When integrating the determinants we therefore find that the first $n$ of them will vanish, two of their columns consisting of proportional elements, whereas the integral of the last determinant is

$$
N\left|\begin{array}{ccccc}
m_{n} & m_{0} & m_{1} & \ldots \ldots & m_{n-1} \\
m_{n+1} & m_{1} & m_{2} & \ldots \ldots & m_{n} \\
m_{n+2} & m_{2} & m_{3} & \ldots \ldots & m_{n+1} \\
\vdots & \vdots & \vdots & & \vdots \\
m_{2 n} & m_{n} & m_{n+1} & \ldots . . & m_{2 n-1}
\end{array}\right|=(-1)^{n} N \Delta_{1,1} .
$$




\section{Choice in the Distribution of Observations}

As $\Delta_{1, n+2, n+2,1}=-\Delta_{n+2, n+2,1,1}$, the integral of the last term of (11) equals $N$. The integration of the other terms, including the first, gives the same result so that

$$
\int_{n} \sigma_{y}^{2} \frac{\psi(x)}{f(x)} d x=\sigma^{2}(n+1)
$$

and as

$$
\int \frac{\psi(x)}{f(x)} d x=N m_{0}
$$

the mean value of ${ }_{n} \sigma_{y}^{2}$ calculated in this special way is

$$
\overline{\sigma^{2}} \cdot \frac{(n+1)}{m_{0}} \text {. }
$$

It is therefore clear either that ${ }_{n} \sigma_{y}^{2}$ must at all the places of observation be equal to $\frac{\sigma^{2}}{N} \cdot \frac{n+1}{m_{0}}$ or ${ }_{n} \sigma_{y}^{2}$ must at some of these places be greater. The first case cannot be realised by a distribution of which any part is continuous, as ${ }_{n} \sigma_{y}^{2}$ is proved to be of the $2 n$th degree in $x$. If therefore we could find a distribution consisting of groups of observations for which at all the places of observation ${ }_{n} \sigma_{y}^{2}$ was equal to $\frac{\sigma^{2}}{N} \cdot \frac{n+1}{m_{0}}$, and if further we could choose the places of observation so that ${ }_{n} \sigma_{y}^{2}$ at all other places within the range of observations was smaller than that value, we should know that no other distribution of observations with that value for $m_{0}$ could provide a curve of standard deviation with a lower maximum.

If the standard deviation of the observations be constant and equal $\sigma, f(x)$ equals 1 , and so does $m_{0}$. $\Lambda$ fter what we have just proved the maximum of the ${ }_{n} \sigma_{y}^{2}$ curve cannot then be lower than $\frac{\sigma^{2}}{N}(n+1)$. Now when we choose to distribute our $N$ observations in $(n+1)$ equally big groups the adjusted $y$ at each of these $(n+1)$ places will be the mean of the observations and its squared standard deviation will be $\frac{\sigma^{2}}{N}(n+1)$. Hence our problem is reduced to find out how to arrange a table of $(n+1)$ values of a function of the $n$th degree to make the squared standard deviation of any interpolation result inside the range smaller than the squared standard deviation of the values of the table. It will be seen in what follows that this can up to $n$ equal 6 -that is so far as the problem here has been investigated-be obtained by one and only one form of grouping.

When the standard deviation of the observations varies over the range, $m_{0}$ varies with the different distributions, and we cannot use the same method for finding the best distribution. It even appears that the best distribution has not always its maxima at the places of observation.

(8) A second problem which we want to consider here is the condition for two adjusted $y$ 's being uncorrelated. In the beginning of this section it has been shown that the adjusted $y$,

$$
y_{r}=\frac{1}{N} S\left\{\begin{array}{c}
y_{p} \\
f\left(x_{p}\right)
\end{array}\left[\alpha_{0}+\alpha_{1} x_{p}+\alpha_{2} x_{p}^{2}+\ldots \ldots+\alpha_{n} x_{p}^{n}\right]\right\},
$$




\section{KiRstine Smith}

when

$$
\begin{aligned}
& a_{0} m_{0}+a_{1} m_{1}+a_{2} m_{2}+\ldots \ldots+\alpha_{n} m_{n}=1 \\
& \alpha_{0} m_{1}+\alpha_{1} m_{2}+\alpha_{2} m_{3}+\ldots \ldots+\alpha_{n} m_{n+1}=x_{r} \\
& a_{0} m_{2}+\alpha_{1} m_{3}+\alpha_{2} m_{4}+\ldots \ldots+\alpha_{n} m_{n+2}=x_{r}^{2} \\
& \begin{array}{llll}
\vdots & \vdots & \vdots & \vdots
\end{array} \\
& \left.\alpha_{0} m_{n}+\alpha_{1} m_{n+1}+\alpha_{2} m_{n+2}+\ldots . .+\alpha_{n} m_{2 n}=x_{r}^{n}\right)
\end{aligned}
$$

Let $y_{s}$ be another adjusted value, then

where

$$
y_{s}=\frac{1}{N} S\left\{\frac{y_{p}}{f\left(x_{p}\right)}\left[\gamma_{0}+\gamma_{1} x_{p}+\gamma_{2} x_{p}^{2}+\ldots \ldots+\gamma_{n} x_{p}^{n}\right]\right\},
$$

$$
\begin{aligned}
& \gamma_{0} m_{0}+\gamma_{1} m_{1}+\gamma_{2} m_{2}+\ldots \ldots+\gamma_{n} m_{n}=1 \\
& \gamma_{0} m_{1}+\gamma_{1} m_{2}+\gamma_{2} m_{3}+\ldots \ldots+\gamma_{n} m_{n+1}=x_{s} \\
& \gamma_{0} m_{2}+\gamma_{1} m_{3}+\gamma_{2} m_{4}+\ldots . .+\gamma_{n} m_{n+2}=x_{s}^{2}
\end{aligned}
$$

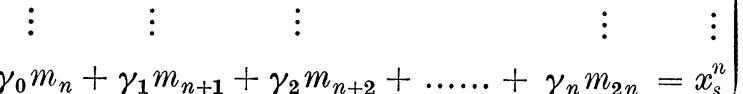

Hence the condition that $y_{r}$ and $y_{s}$ are uncorrelated is, since the squared standard deviation of the observed $y_{p}$ equals $\sigma^{2} f\left(x_{p}\right)$,

$$
\begin{aligned}
& S\left\{\frac{1}{f\left(x_{p}\right)}\left[\alpha_{0}+\alpha_{1} x_{p}+\alpha_{2} x_{p}^{2}+\ldots \ldots+\alpha_{n} x_{p}^{n}\right] \cdot\left[\gamma_{0}+\gamma_{1} x_{p}+\gamma_{2} x_{p}^{2}+\ldots \ldots+\gamma_{n} x_{p}^{n}\right]\right\}=0, \\
& \text { or } S\left\{\frac{\gamma_{0}}{f\left(x_{p}\right)}\left[\alpha_{0}+\alpha_{1} x_{p}+\alpha_{2} x_{p}^{2}+\ldots \ldots+\alpha_{n} x_{p}^{n}\right]\right\} \\
& +S\left\{\frac{\gamma_{1}}{f\left(x_{p}\right)}\left[\alpha_{0} x_{p}+\alpha_{1} x_{p}^{2}+\alpha_{2} x_{p}^{3}+\ldots \ldots+\alpha_{n} x_{p}^{n+1}\right]\right\} \\
& +S\left\{\frac{\gamma_{2}}{f\left(x_{p}\right)}\left[\alpha_{0} x_{p}^{2}+\alpha_{1} x_{p}^{3}+\alpha_{2} x_{p}^{4}+\ldots \ldots+\alpha_{n} x_{p}^{n+2}\right]\right\}+\ldots \ldots \\
& +S\left\{\frac{\gamma_{n}}{f\left(x_{p}\right)}\left[\alpha_{0} x_{p}^{n}+\alpha_{1} x_{p}^{n+1}+\alpha_{2} x_{p}^{n+2}+\ldots \ldots+\alpha_{n} x_{p}^{2 n}\right]\right\}=0 .
\end{aligned}
$$

Remembering that $S\left\{\frac{x_{p}^{q}}{f\left(x_{p}\right)}\right\}=N m_{q}$ and applying the relations (16) this reduces to

$$
\gamma_{0}+\gamma_{1} x_{r}+\gamma_{2} x_{r}^{2}+\ldots \ldots+\gamma_{n} x_{r}^{n}=0,
$$

from which the $\gamma^{\prime}$ s are eliminated by (17).

$$
\left|\begin{array}{cccccc}
0 & 1 & x_{r} & x_{r}^{2} & \ldots \ldots & x_{r}^{n} \\
1 & m_{0} & m_{1} & m_{2} & \ldots \ldots & m_{n} \\
x_{s} & m_{1} & m_{2} & m_{3} & \ldots \ldots & m_{n+1} \\
x_{s}^{2} & m_{2} & m_{3} & m_{4} & \ldots \ldots & m_{n+2} \\
\vdots & \vdots & \vdots & \vdots & & \vdots \\
x_{s}^{n} & m_{n} & m_{n+1} & m_{n+2} & \ldots . & m_{2 n}
\end{array}\right|=0
$$

is therefore the condition that $y_{r}$ and $y_{s}$ are uncorrelated. 


\section{Choice in the Distribution of Observations}

(9) Returning to the formula (11) for $\sigma_{y}^{2}$ written as a sum of squares we shall now prove that the $(p+1)$ st term of this put equal to zero determines a set of $p$ abscissae the adjusted $y$ 's of which are mutually uncorrelated both for a function of the pth and the $(p-1)$ st degree.

The condition for $y_{1}$ and $y_{2}$ corresponding to the arguments $x_{1}$ and $x_{2}$ being uncorrelated is for a function of the $(p-1)$ st degree

$$
\left|\begin{array}{cccccc}
0 & 1 & x_{1} & x_{1}^{2} & \ldots \ldots & x_{1}^{p-1} \\
1 & m_{0} & m_{1} & m_{2} & \ldots \ldots & m_{p-1} \\
x_{2} & m_{1} & m_{2} & m_{3} & \ldots \ldots & m_{p} \\
x_{2}^{2} & m_{2} & m_{3} & m_{4} & \ldots \ldots & m_{p+1} \\
\vdots & \vdots & \vdots & \vdots & & \vdots \\
x_{2}^{p-1} & m_{p-1} & m_{p} & m_{p+1} & \ldots \ldots & m_{2 p-2}
\end{array}\right|=0,
$$

and for the same distribution of observations and for a function of the $p$ th degree the condition is

Putting

$$
\left|\begin{array}{cccccc}
0 & 1 & x_{1} & x_{1}^{2} & \ldots \ldots & x_{1}^{p} \\
1 & m_{0} & m_{1} & m_{2} & \ldots \ldots & m_{p} \\
x_{2} & m_{1} & m_{2} & m_{3} & \ldots \ldots & m_{p+1} \\
x_{2}^{2} & m_{2} & m_{3} & m_{4} & \ldots \ldots & m_{p+2} \\
\vdots & \vdots & \vdots & \vdots & & \vdots \\
x_{2}^{p} & m_{p} & m_{p+1} & m_{p+2} & \ldots & m_{2 p}
\end{array}\right|=0 .
$$

these conditions may be written

$$
\left|\begin{array}{ccccc}
m_{0} & m_{1} & m_{2} & \ldots \ldots & m_{p} \\
m_{1} & m_{2} & m_{3} & \ldots \ldots & m_{p+1} \\
m_{2} & m_{3} & m_{4} & \ldots \ldots & m_{p+2} \\
\vdots & \vdots & \vdots & & \vdots \\
m_{p} & m_{p+1} & m_{p+2} & \ldots \ldots & m_{2 p}
\end{array}\right|=D,
$$

$$
\sum_{0}^{p-1}\left\{x_{1}^{r} \cdot x_{2}^{s} D_{p+1, p+1, r+1, s+1}\right\}=0
$$

and

$$
\sum_{0}^{p}\left\{x_{1}^{r} \cdot x_{2}^{s} D_{r+\mathbf{1}, s+1}\right\}=0
$$

where the sums include all combinations of powers with $r$ and $s$ lying between 0 and $(p-1)$, and 0 and $p$ respectively.

Now we have for an orthosymmetrical determinant $\Delta$,

$$
\Delta_{s s} \cdot \Delta_{s^{\prime} s^{\prime \prime}}-\Delta . \Delta_{s s s^{\prime} s^{\prime \prime}}=\Delta_{s s^{\prime}} \cdot \Delta_{s s^{\prime \prime}}
$$

If therefore (19) is multiplied by $D$ and subtracted from (20) multiplied by $D_{p+1, p+1}$ the coefficient of $x_{1}^{r} \cdot x_{2}^{s}$ becomes

$$
D_{p+1, p+1} . D_{r+1, s+1}-D . D_{p+1, p+1, r+1, s+1}=D_{p+1, r+1} \cdot D_{p+1, s+1},
$$

as long as both $r$ and $s$ are smaller than $p$. 
When one of them, for example $s$, equals $p$ the term is

$$
x_{1}^{r} \cdot x_{2}^{p} \cdot D_{p+1, p+1} \cdot D_{r+1, p+1},
$$

which is of the same form and this also holds for $r=s=p$ when the term is

The total result is thus

$$
x_{1}^{p} \cdot x_{2}^{p} \cdot D_{p+1, n+1}^{2} \text {. }
$$

$$
\sum_{0}^{p} x_{1}^{r} \cdot x_{2}^{s} D_{p+1, r+1} \cdot D_{p+1, s+1}=0,
$$

or in the form of determinants

$$
\left|\begin{array}{cccccc}
1 & m_{0} & m_{1} & m_{2} & \ldots \ldots & m_{p-1} \\
x_{1} & m_{1} & m_{2} & m_{3} & \ldots \ldots & m_{p} \\
x_{1}^{2} & m_{2} & m_{3} & m_{4} & \ldots \ldots & m_{p+1} \\
\vdots & \vdots & \vdots & \vdots & & \vdots \\
x_{1}^{p} & m_{p} & m_{p+1} & m_{p+2} & \ldots \ldots & m_{2 p-1}
\end{array}\right| \cdot\left|\begin{array}{cccccc}
1 & m_{0} & m_{1} & m_{2} & \ldots \ldots & m_{p-1} \\
x_{2} & m_{1} & m_{2} & m_{3} & \ldots \ldots & m_{p} \\
x_{2}^{2} & m_{2} & m_{3} & m_{4} & \ldots \ldots & m_{p+1} \\
\vdots & \vdots & \vdots & \vdots & & \vdots \\
x_{2}^{p} & m_{p} & m_{p+1} & m_{p+2} & \ldots \ldots & m_{2 p-1}
\end{array}\right|=0 .
$$

Hence $x_{1}$ and $x_{2}$ must be roots of

$$
\left|\begin{array}{cccccc}
1 & m_{0} & m_{1} & m_{2} & \ldots \ldots & m_{p-1} \\
x & m_{1} & m_{2} & m_{3} & \ldots \ldots & m_{p} \\
x^{2} & m_{2} & m_{3} & m_{4} & \ldots \ldots & m_{p+1} \\
\vdots & \vdots & \vdots & \vdots & & \vdots \\
x^{p} & m_{p} & m_{p+1} & m_{p+2} & \ldots . & m_{2 p-1}
\end{array}\right|=0 \quad \ldots \ldots \ldots \ldots \ldots(21) .
$$

When $x_{1}$ is found from this and substituted in (19) or (20) we get since the coefficient of $x_{2}^{\prime \prime}$ in the latter is zero an equation of the $(p-1)$ st degree to determine $x_{2}$. It is therefore clear that any pair of roots of (21) determine a pair of uncorrelated $y$ 's.

II. The "best" grouping of observations with constant standard deviation.

(1) It was shown in the last section under (7) that the mean of the squared standard deviations of the adjusted $y$ taken over the places of observation and weighted with the number of observations at each place is equal to $\frac{\sigma^{2}}{N}(n+1)$ and that therefore the curve of squared standard deviation can never be entirely below that value. And further, that since $(n+1)$ equally big groups of observations at the places of observations give the squared standard deviation this minimum, there is the possibility, ${ }_{n} \sigma_{y}^{2}$ being of the $2 n$th degree in $x$, that by placing the groups at special positions the curve of squared standard deviation could have those values $\frac{\sigma^{2}}{N}(n+1)$ as its maxima within the range of observations.

Let $x_{1}, x_{2} \ldots x_{p} \ldots x_{n+1}$ be the places of observations and $\bar{y}_{x_{p}}$ the mean of the observations at $x_{p}$, the interpolation formula of Lagrange is then

$$
y=\Sigma\left\{\frac{\left(x-x_{1}\right)\left(x-x_{2}\right) \ldots \ldots\left(x-x_{n+1}\right)}{\left(x_{p}-x_{1}\right)\left(x_{p}-x_{2}\right) \ldots \ldots\left(x_{p}-x_{n+1}\right)} \bar{y}_{x_{n}}\right\},
$$

the sum taken over all the places of observation. 
From this we find

$$
\sigma_{y}^{2}=\frac{\sigma^{2}}{N}(n+1) \Sigma\left\{\frac{\left(x-x_{1}\right)\left(x-x_{2}\right) \ldots \ldots\left(x-x_{n+1}\right)}{\left(x_{p}-x_{1}\right)\left(x_{p}-x_{2}\right) \ldots \ldots\left(x_{p}-x_{n+1}\right)}\right\}^{2}
$$

which for $x=x_{1}, x_{2} \ldots x_{n+1}$ equals $\frac{\sigma^{2}}{N}(n+1)$, the $n$ terms of the sum being zero and the $(n+1)$ st taking the value 1 as it ought to. If $x_{p}$ be the greatest of the $x$ 's it is hence clear that for $x>x_{p}$, since

$$
\begin{gathered}
\left\{\frac{\left(x-x_{1}\right)\left(x-x_{2}\right) \ldots \ldots\left(x-x_{n+1}\right)}{\left(x_{p}-x_{1}\right)\left(x_{p}-x_{2}\right) \ldots \ldots\left(x_{p}-x_{n+1}\right)}\right\}^{2}>1, \\
\sigma_{y}^{2}>\frac{\sigma^{2}}{N}(n+1) .
\end{gathered}
$$

The same applies to any $x$ smaller than the smallest of the places of observation. Therefore as we want $\sigma_{y}^{2}$ to be $₹ \frac{\sigma^{2}}{N}(n+1)$ at the ends of the range we have to place two of our groups of observations there.

Let us take the half of the range within which it is possible to make observations as the unit of $x$ so that the range goes from -1 to 1 .

(2) Hence for a linear function there is no choice left, the two groups of observations must be at -1 and 1 .

According to (22) we have

or

$$
\begin{aligned}
& { }_{1} \sigma_{y}^{2}=\frac{\sigma^{2}}{N} \cdot 2\left\{\frac{(x+1)^{2}}{4}+\frac{(x-1)^{2}}{4}\right\} \\
& { }_{1} \sigma_{y}^{2}=\frac{\sigma^{2}}{N} \cdot 2\left\{1-\frac{1}{2}\left(1-x^{2}\right)\right\},
\end{aligned}
$$

which illustrate the well-known fact that by simple interpolation between two equally good values of a table, we obtain interpolated values with less probable error than those of the table.

(3) Investigating a function of the second degree we have a third group to place besides the two at -1 and 1 , that is if we do not beforehand suppose the distribution to be symmetrical. Let the third group be at $\alpha$, then the interpolation gives

from which

$$
y=\frac{(x-1)(x-\alpha)}{2(1+\alpha)} \bar{y}_{-1}+\frac{(x+1)(x-\alpha)}{2(1-\alpha)} \bar{y}_{1}+\frac{x^{2}-1}{\alpha^{2}-1} \bar{y}_{\alpha},
$$

$$
{ }_{2} \sigma_{y}^{2}=\frac{\sigma^{2}}{N} \cdot 3\left\{\left[\frac{(x-1)(x-\alpha)}{2(1+\alpha)}\right]^{2}+\left[\frac{(x+1)(x-\alpha)}{2(1-\alpha)}\right]^{2}+\left[\frac{x^{2}-1}{\alpha^{2}-1}\right]^{2}\right\} .
$$

We want this to be a maximum for $x=a$, but $\left(\frac{d \sigma_{y}^{2}}{d x}\right)_{x=a}$ can only vanish for $\alpha=0$, in which case $\sigma_{y}^{2}$ is reduced to

$$
{ }_{2} \sigma_{y}^{2}=\frac{\sigma^{2}}{N} \cdot 3\left\{1-\frac{3}{2} x^{2}\left(1-x^{2}\right)\right\},
$$


which shows that we have succeeded in making $\sigma_{y}^{2}$ a maximum at $x=0$ and obtained a standard deviation with the maximum value $\frac{\sigma^{2}}{N} 3$, as we desired.

(4) For a function of the third degree we find from four groups of observations at $-1,1, \alpha$ and $\gamma$ that

$$
\begin{aligned}
y=\frac{(x-1)(x-a)(x-\gamma)}{-2(1+\alpha)(1+\gamma)} \bar{y}_{-1} & +\frac{(x+1)(x-a)(x-\gamma)}{2(1-\alpha)(1-\gamma)} \bar{y}_{1} \\
& +\frac{\left(x^{2}-1\right)(x-\gamma)}{\left(\alpha^{2}-1\right)(\alpha-\gamma)} \bar{y}_{\alpha}+\frac{\left(x^{2}-1\right)(x-\alpha)}{\left(\gamma^{2}-1\right)(\gamma-\alpha)} \bar{y}_{\gamma}
\end{aligned}
$$

and

$$
\begin{aligned}
{ }_{3} \sigma_{y}^{2}=\frac{\sigma^{2}}{N} \cdot 4\left\{\left[\frac{(x-1)(x-\alpha)(x-\gamma)}{2(1+\alpha)(1+\gamma)}\right]^{2}+\left[\frac{(x+1)(x-\alpha)(x-\gamma)}{2(1-\alpha)(1-\gamma)}\right]^{2}\right. \\
\left.+\left[\frac{\left(x^{2}-1\right)(x-\gamma)}{\left(1-\alpha^{2}\right)(\alpha-\gamma)}\right]^{2}+\left[\frac{\left(x^{2}-1\right)(x-\alpha)}{\left(1-\gamma^{2}\right)(\alpha-\gamma)}\right]^{2}\right\} .
\end{aligned}
$$

The condition

$$
\left(\frac{d \sigma_{y}^{2}}{d x}\right)_{x=a}=0
$$

requires

$$
3 a^{2}-2 \alpha \gamma-1=0 \text {, }
$$

and

$$
\left(\frac{d \sigma_{y}^{2}}{d x}\right)_{x=\gamma}=0
$$

requires

from which is got

$$
\begin{gathered}
3 \gamma^{2}-2 \alpha \gamma-1=0, \\
\alpha^{2}=\gamma^{2},
\end{gathered}
$$

and, since $\alpha \gtrless \gamma$,

$$
\alpha^{2}=\gamma^{2}=\frac{1}{5} \text {. }
$$

By introducing this value for $\alpha^{2}$ and $\gamma^{2}$ in $\sigma_{y /}^{2}$ we find

$$
{ }_{3} \sigma_{y}^{2}=\frac{\sigma^{2}}{N} \cdot 4\left\{1-\frac{3.5^{2}}{2^{4}}\left(x^{2}-\frac{1}{5}\right)^{2}\left(1-x^{2}\right)\right\},
$$

which has the required maxima at $\pm \sqrt{ } \frac{1}{5}$.

(5) For the functions of higher degree we shall at once assume that the distributions sought are symmetrical, since it is pretty clear from the symmetry of $y$ and $\sigma_{y}^{2}$ with regard to the sought positions that it must be so.

To determine a function of the fourth degree let us put groups of observations at $\pm 1, \pm \alpha$ and 0 . The expression for $\sigma_{y}^{2}$ can be written down at once and is such that the terms arising from the groups at +1 and -1 can be put together as well as the terms from $+\alpha$ and $-\alpha$, then

$$
\begin{gathered}
{ }_{4} \sigma_{y}^{2}=\frac{\sigma^{2}}{N} \cdot 5\left[\left\{\frac{\left(x^{2}-1\right)\left(x^{2}-\alpha^{2}\right)}{\alpha^{2}}\right\}^{2}+\frac{1}{2}\left\{\frac{x\left(x^{2}-\alpha^{2}\right)}{1-\alpha^{2}}\right\}^{2}\left(x^{2}+1\right)+\frac{1}{2}\left\{\frac{x\left(x^{2}-1\right)}{\alpha^{2}\left(1-\alpha^{2}\right)}\right\}^{2}\left(x^{2}-\alpha^{2}\right)\right] . \\
\left(\frac{d \sigma_{y}^{2}}{d x}\right)_{x=a}=0 \text { provides the condition } \frac{3-7 \alpha^{2}}{\alpha\left(1-\alpha^{2}\right)}=0 \text { or } \\
\alpha^{2}=\frac{3}{7},
\end{gathered}
$$

with which value the squared standard deviation becomes

$$
{ }_{4} \sigma_{y}^{2}=\frac{\sigma^{2}}{N} \cdot 5\left\{1-\frac{5 \cdot 7^{2}}{2^{4}} x^{2}\left(x^{2}-\frac{3}{7}\right)^{2}\left(1-x^{2}\right)\right\},
$$

which has the required characteristics. 


\section{Choice in the Distribution of Observations}

(6) Adjusting by a function of the fifth degree six equally big groups of observations at the arguments $\pm 1, \pm \alpha$ and $\pm \gamma$ the squared standard deviation of the adjusted $y$ is

$$
\begin{aligned}
{ }_{5} \sigma_{y}^{2}=\frac{\sigma^{2}}{N} \cdot 6\left\{\frac{1}{2}\left[\frac{\left(x^{2}-\alpha^{2}\right)\left(x^{2}-\gamma^{2}\right)}{\left(1-\alpha^{2}\right)\left(1-\gamma^{2}\right)}\right]^{2}\left(x^{2}+1\right)\right. & +\frac{1}{2}\left[\frac{\left(x^{2}-\gamma^{2}\right)\left(x^{2}-1\right)}{\alpha\left(1-\alpha^{2}\right)\left(\alpha^{2}-\gamma^{2}\right)}\right]^{2}\left(x^{2}+\alpha^{2}\right) \\
& \left.+\frac{1}{2}\left[\frac{\left(x^{2}-1\right)\left(x^{2}-\alpha^{2}\right)}{\gamma\left(1-\gamma^{2}\right)\left(\alpha^{2}-\gamma^{2}\right)}\right]^{2}\left(x^{2}+\gamma^{2}\right)\right\} .
\end{aligned}
$$

The condition for maximum at $x= \pm \alpha$ is

$$
9 a^{4}-5 \alpha^{2} \gamma^{2}-5 \alpha^{2}+\gamma^{2}=0
$$

which together with the condition for maximum at $x= \pm \gamma$

$$
9 \gamma^{4}-5 \alpha^{2} \gamma^{2}-5 \gamma^{2}+\alpha^{2}=0
$$

since $\alpha^{2}$ must be $\gtrless \gamma^{2}$ results in

or

$$
\begin{aligned}
& \alpha^{2}+\gamma^{2}=\frac{2}{3} \text { and } \alpha^{2} \gamma^{2}=\frac{1}{21} \\
& \left.\begin{array}{l}
\alpha^{2} \\
\gamma^{2}
\end{array}\right\}=\frac{7 \pm 2 \sqrt{ } 7}{21} .
\end{aligned}
$$

When these values are substituted in the expression above for $\sigma_{y}^{2}$ this may by somewhat lengthy algebraic operations be brought into the form

$$
{ }_{5} \sigma_{y}^{2}=\frac{\sigma^{2}}{N} \cdot 6\left\{1-\frac{3^{3} \cdot 5 \cdot 7^{2}}{2^{7}}\left(x^{2}-\alpha^{2}\right)^{2}\left(x^{2}-\gamma^{2}\right)^{2}\left(1-x^{2}\right)\right\} .
$$

(7) For a function of the sixth degree the observations may be supposed to be at $\pm 1, \pm \alpha, \pm \gamma$ and 0 .

The expression for the squared standard deviation of an adjusted $y$ becomes

$$
\begin{aligned}
{ }_{6} \sigma_{y}^{2}=\frac{\sigma^{2}}{N} \cdot 7\left\{\left[\frac{\left(x^{2}-\alpha^{2}\right)\left(x^{2}-\gamma^{2}\right)\left(x^{2}-1\right)}{a^{2} \gamma^{2}}\right]^{2}+\frac{1}{2}\left[\frac{x\left(x^{2}-\gamma^{2}\right)\left(x^{2}-1\right)}{\alpha^{2}\left(\alpha^{2}-\gamma^{2}\right)\left(\alpha^{2}-1\right)}\right]^{2}\left(x^{2}+\alpha^{2}\right)\right. \\
\left.+\frac{1}{2}\left[\frac{x\left(x^{2}-\alpha^{2}\right)\left(x^{2}-1\right)}{\gamma^{2}\left(\alpha^{2}-\gamma^{2}\right)\left(\gamma^{2}-1\right)}\right]^{2}\left(x^{2}+\gamma^{2}\right)+\frac{1}{2}\left[\frac{x\left(x^{2}-\alpha^{2}\right)\left(x^{2}-\gamma^{2}\right)}{\left(\alpha^{2}-1\right)\left(\gamma^{2}-1\right)}\right]^{2}\left(x^{2}+1\right)\right\} .
\end{aligned}
$$

A maximum at $x= \pm \alpha$ requires

$$
11 \alpha^{4}-7 \alpha^{2} \gamma^{2}-7 \alpha^{2}+3 \gamma^{2}=0
$$

and a maximum at $x= \pm \gamma$ requires

$$
11 \gamma^{4}-7 \alpha^{2} \gamma^{2}-7 \gamma^{2}+3 \alpha^{2}=0
$$

which added and subtracted provide

and

$$
\begin{gathered}
11\left(\alpha^{2}+\gamma^{2}\right)^{2}-36 \alpha^{2} \gamma^{2}-4\left(\alpha^{2}+\gamma^{2}\right)=0, \\
\left(\alpha^{2}-\gamma^{2}\right)\left\{11\left(\alpha^{2}+\gamma^{2}\right)-10\right\}=0 .
\end{gathered}
$$

Since we must have $\alpha^{2} \lesseqgtr \gamma^{2}$,

$$
\begin{aligned}
& \alpha^{2}+\gamma^{2}=\frac{10}{11} \text { and } a^{2} \gamma^{2}=\frac{5}{33} \text {, } \\
& \left.\begin{array}{l}
a^{2} \\
\gamma^{2}
\end{array}\right\}=\frac{15 \pm 2 \sqrt{ } 15}{33} .
\end{aligned}
$$$$
\text { or }
$$

The expression for $\sigma_{y}^{2}$ may after rather laborious operations be brought into the form

$$
{ }_{6} \sigma_{y}^{2}=\frac{\sigma^{2}}{N} \cdot 7\left\{1-\frac{3^{3} \cdot 7 \cdot 11^{2}}{2^{7}} x^{2}\left(x^{2}-\alpha^{2}\right)^{2}\left(x^{2}-\gamma^{2}\right)^{2}\left(1-x^{2}\right)\right\} .
$$


(8) It is thus, as we aimed at, shown for functions up to the sixth degree that by distributing the observations in $(n+1)$ equally big groups and choosing the places of these groups in one special way we can manage to keep the standard deviation of any adjusted $y$ within the possible range of observations less than the standard deviation at the places of observation. There is every reason to believe that the rule holds for any degree of function, but as the general proof would be very complicated and as almost all practical cases will be covered by functions up to the sixth degree, the problem can therefore be left at this stage.

As we have proved, any other distribution of observations leads to a curve of squared standard deviation that has a higher maximum value within the range. This special set of $(n+1)$ groups has therefore a very conspicuous advantage over all other distributions of observations. The application of it is however limited in that it demands that the degree of the function must be known beforehand and thus the observations do not provide any justification for the form of function chosen. If however the function has been fully investigated beforehand and there is no doubt about its form, $(n+1)$ equally big groups of observations placed as indicated are the most desirable set of observations possible. The approximate values of the places of the groups are given in the table below.

TABLE I.

\begin{tabular}{|c|c|c|c|c|c|c|}
\hline \multirow[t]{2}{*}{ Degree of function } & Ist & 2nd & $3 \mathrm{rd}$ & 4th & 5th & $\begin{array}{c}6 \text { th } \\
1.0000\end{array}$ \\
\hline & 1.0000 & 1.0000 & 1.0000 & 1.0000 & 1.0000 & 1.0000 \\
\hline \multirow{3}{*}{$\begin{array}{l}\text { Places of } \\
\text { observation }\end{array}$} & - & $\cdot 0000$ & · 4472 & 6547 & $\cdot 7651$ & •8302 \\
\hline & - & - & - & .0000 & .2852 & •4689 \\
\hline & - & - & - & - & - & .0000 \\
\hline
\end{tabular}

With rougher approximation the intervals between the observations, still expressed by the half range as unit, are as follows:

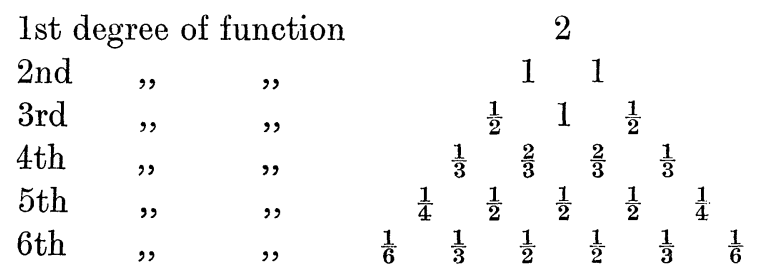

The six curves of standard deviation are represented in Diagram 1. It will be seen that the minima of a curve, if it has more than two, are the lower the greater their distances from the middle of the range, so that the variation of the standard deviation is greatest in the outermost intervals of the range.

III. Uniform continuous distribution of observations with constant standard deviation. General formulae.

(1) As was pointed out in the last section the lumping up of observations in groups just necessary to determine the constants of the function in question has some drawbacks and cannot be recommended as a universal rule. In many cases it is through the observations themselves that we first get to know the form of the 


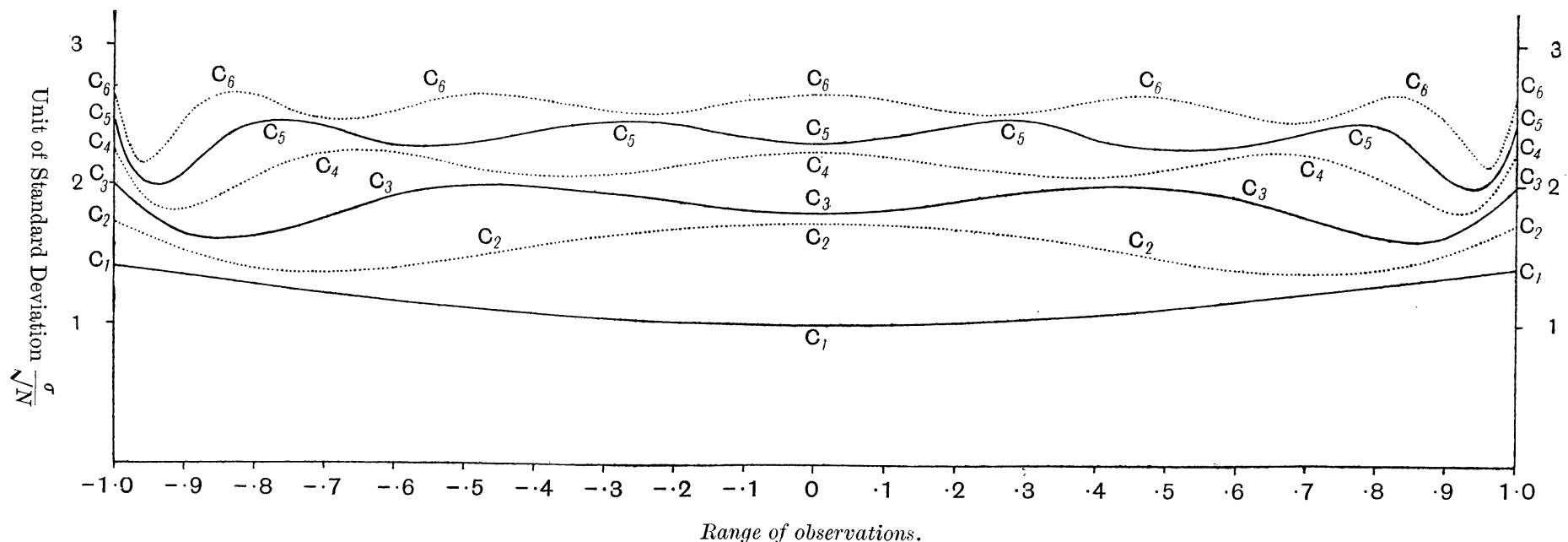

$\mathrm{C}_{1} \mathrm{C}_{1}$. Function of First Degree

$\begin{array}{lll}\mathrm{C}_{2} \mathrm{C}_{2} . & , & \text { Second , } \\ \mathrm{C}_{3} \mathrm{C}_{3} . & , & \text { Third ," } \\ \mathrm{C}_{4} \mathrm{C}_{4} . & , & \text { Fourth , } \\ \mathrm{C}_{5} \mathrm{C}_{5} . & , & \text { Fifth , } \\ \mathrm{C}_{6} \mathrm{C}_{6} . & , & \text { Sixth , }\end{array}$

Diagram 1. Curves of Standard Deviations. Equally big clusters at definite points. 
function, and thus a full investigation may require more groups of observations than merely a number equal to the assumed number of constants in the formula. Besides, even when we believe we know on theoretical or other grounds beforehand the nature of the function a priori we may consider it prudent to distribute the observations so that they supply us with data whereby we may control our hypothesis that the assumed function is the right one.

It is therefore desirable to find other forms of distributions which, at the same time as they make the standard deviation of the adjusted function vary little inside the range of observations, are more uniformly spread over this range.

(2) A uniform continuous distribution at once recommends itself as the simplest assumption. As we suppose the observations to have constant standard deviations the elements of the determinants of (15) are the moment coefficients of the $x$ 's at the places of observation.

When the $N$ observations are uniformly spread between $x=-1$ and $x=1$,

$$
\mu_{2 r}=\frac{1}{2 r+1} \text { and } \mu_{2 r+1}=0,
$$

and the expression for ${ }_{2 p} \sigma_{y}^{2}$ is, according to (15),

$$
\begin{aligned}
& { }_{2 p} \sigma_{y}^{2}=\frac{\sigma^{2}}{N}\left\{1+\frac{x^{2}}{\mu_{2}}+\frac{\left|\begin{array}{cc}
1 & 1 \\
x^{2} & \mu_{2}
\end{array}\right|^{2}}{1 .\left|\begin{array}{rr}
1 & \mu_{2} \\
\mu_{2} & \mu_{4}
\end{array}\right|}+x^{2} \frac{\left|\begin{array}{cc}
1 & \mu_{2} \\
x^{2} & \mu_{4}
\end{array}\right|^{2}}{\mu_{2} \cdot\left|\begin{array}{ll}
\mu_{2} & \mu_{4} \\
\mu_{4} & \mu_{6}
\end{array}\right|}+\ldots \ldots\right.
\end{aligned}
$$

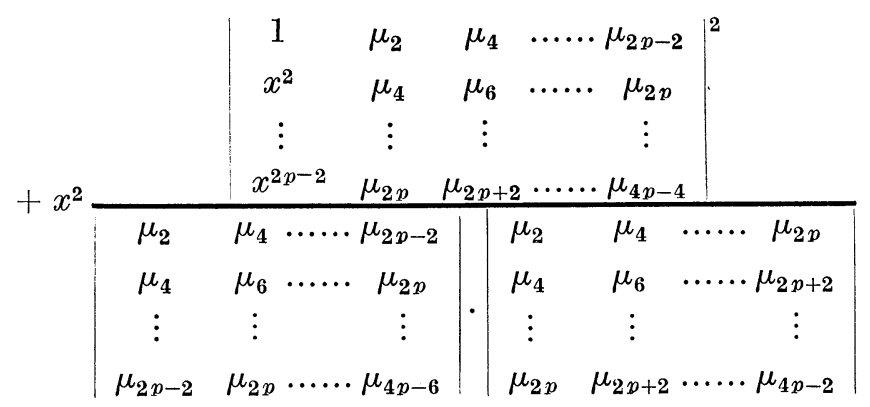

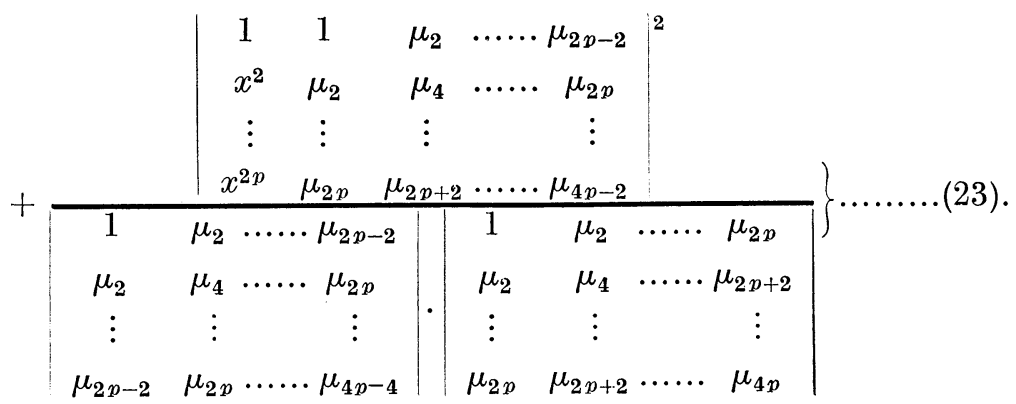

By this formula we may evaluate successively ${ }_{1} \sigma_{y}^{2},{ }_{2} \sigma_{y}^{2} \ldots{ }_{2 p} \sigma_{y}^{2}$ when we know the two general terms of which the sum consists. 
(3) The determinant of the order $p$,

$$
{ }_{p}^{q} \Delta=\left|\begin{array}{cccc}
1 & \frac{1}{2 q+1} & \cdots \cdots & \frac{1}{2 q+2 p-3} \\
2 q-1 & 1 & \cdots & \frac{1}{2 q+2 p-1} \\
\frac{1}{2 q+1} & \frac{1}{2 q+3} & \cdots \cdots & \vdots \\
\vdots & \vdots & 1 & \frac{1}{2 q+4 p-5}
\end{array}\right|,
$$

which includes the two types of the denominators in (23), shall first be evaluated.

$$
\text { We find } \quad{ }_{1} \stackrel{q}{\Delta}=\frac{1}{2 q-1} \text { and }{ }_{2} \stackrel{q}{\Delta}=\frac{2^{2}}{(2 q-1)(2 q+1)^{2}(2 q+3)},
$$

and it shall be proved that if

$$
{ }_{p} \stackrel{q}{\Delta}=\left\{1^{p-1} \cdot 2^{p-2} \ldots \ldots(p-2)^{2}(p-1)\right\}^{2} \cdot 2^{p(p-1)}{ }_{p} \stackrel{q}{\Pi} \ldots
$$

up to the order $p,{ }_{p} \stackrel{q}{I}$ being the product of the elements of ${ }_{p} \stackrel{q}{\Delta}$, the rule holds for determinants of any order.

It is clear that

and

$$
\begin{aligned}
& { }_{p+1} \stackrel{q}{\Delta}{ }_{1,1}={ }_{p}^{q+2} \Delta, \quad{ }_{p+1}^{q} \stackrel{\Delta}{\Delta}_{p+1, p+1}={ }_{p}^{q} \Delta, \quad{ }_{p+1}^{q}{ }_{1, p}(-1)^{p+1}={ }_{p}^{q+1} \Delta \\
& { }_{p+1}^{q} \stackrel{q}{\Delta}_{p+1, p+1,1,1}=\underset{p-1}{q+2} \Delta .
\end{aligned}
$$

If we therefore in the general relation for an orthosymmetrical determinant

$$
\Delta=\frac{\Delta_{s s} \cdot \Delta_{s^{\prime} s^{\prime}}-\Delta_{s s^{\prime}}^{2}}{\Delta_{s s s^{\prime} s^{\prime}}}
$$

put $s=1$ and $s^{\prime}=p+1$ and $\Delta={ }_{p+1} \stackrel{q}{\Delta}$, we find

and, using (24),

$$
{ }_{p+1} \stackrel{q}{\Delta}=\frac{p_{p}^{q} \Delta \cdot{ }_{p}^{q+2} \Delta-{ }_{p}^{q+1} \Delta^{2}}{\underset{p-1}{q+2} \Delta}
$$

$$
{ }_{p+1} \stackrel{q}{\Delta}^{q}=\frac{\left\{1^{p-1} \cdot 2^{p-2} \ldots \ldots(p-2)^{2}(p-1)\right\}^{4}}{\left\{1^{p-2} \cdot 2^{p-3} \ldots \ldots(p-3)^{2}(p-2)\right\}^{2}} \cdot 2^{(p-1)(p+2)} \cdot \frac{{ }_{p}^{q} \prod^{q} \cdot{ }_{p}^{q+2} \Pi_{p}^{q+1}{ }_{p}^{q+2}}{\prod_{p-1}^{q+2}} .
$$

Now, according to the definition of $\Pi$,

$$
\begin{aligned}
& \frac{{ }_{p} \frac{q}{\Pi}}{{ }_{p+1} \prod} \cdot \frac{{ }_{p}^{q+2} \Pi}{{ }_{p+1} \Pi}=(2 q-1)(2 q+1)^{2}(2 q+3)^{2} \ldots \ldots(2 q+4 p-3)^{2}(2 q+4 p-1) \\
& \frac{{ }_{p}^{q+1} \Pi^{2}}{{ }_{p+1} \Pi^{q}}=(2 q-1)^{2}(2 q+1)^{2}(2 q+3)^{2} \ldots \ldots(2 q+4 p-3)^{2}(2 q+4 p-1)^{2} \\
& \text { and }
\end{aligned}
$$

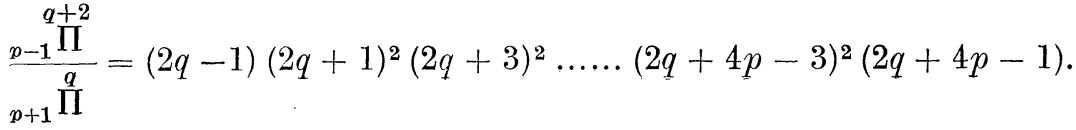




$$
\begin{aligned}
& \text { Hence } \\
& { }_{p+1} \stackrel{q}{\Delta}=\left\{1^{p} \cdot 2^{p-1} \ldots \ldots(p-2)^{3}(p-1)^{2}\right\}^{2} \cdot 2^{(p-1)(p+2)} \cdot{ }_{p+1} \stackrel{q}{\Pi} \cdot\left[(2 q+2 p-1)^{2}\right. \\
& -(2 q-1)(2 q+4 p-1)] \text {, } \\
& { }_{p+1} \stackrel{q}{\Delta}=\left\{1^{p} \cdot 2^{p-1} \ldots \ldots(p-1)^{2} \cdot p\right\}^{2} \cdot 2^{p(p+1)} \cdot{ }_{p+1} \stackrel{q}{\Pi},
\end{aligned}
$$

which agrees with (24).

(4) Next we have to evaluate the minors of ${ }_{p} \stackrel{q}{\Delta}$ necessary for calculating the numerators in (23). For this purpose we only need the minors ${ }_{p} \stackrel{q}{\Delta}_{s, p}$, but to carry through the proof by induction ${ }_{p} \stackrel{q}{\Delta}_{s, r}$ for any values of $s$ and $r$ is needed.

For ${ }_{3} \stackrel{q}{\Delta}_{2,3}$ we directly find,

$$
{ }_{3}^{\stackrel{q}{\Delta}} \Delta_{2,3}=\frac{-2^{2} \cdot 2}{(2 q-1)(2 q+1)(2 q+3)(2 q+5)}
$$

and

$$
{ }_{3} \Delta_{2,2}^{q}=\frac{2^{2} \cdot 2^{2}}{(2 q-1)(2 q+3)^{2}(2 q+7)},
$$

these both agree with the following formula which will be proved by induction, ${ }_{p} \stackrel{q}{\Delta}_{s, r}=(-1)^{r+s} \beta_{p-1, s-1} \cdot \beta_{p-1, r-1}\left\{1^{p-2} \cdot 2^{p-3} \ldots \ldots(p-3)^{2}(p-2)\right\}^{2} \cdot 2^{(p-1)(p-2)}{ }_{p} \stackrel{q}{\Pi}_{s, r}$ $\beta_{p-1, s-1}$ is the binomial coefficient $\frac{\mid p-1}{|s-1| p-s}$ and ${ }_{p} \prod_{s, r}$ the product of all the elements of ${ }_{p} \stackrel{q}{\Delta}, r$.

The relation has to be proved first for $r=s$, then for $r=p$ and finally for any combination $s$ and $r$.

For the first two proofs we use the relation between the minors of an orthosymmetrical determinant

$$
\frac{\Delta_{s s}}{\Delta_{s^{\prime} s^{\prime \prime}}}=\frac{\Delta_{s s s^{\prime} s^{\prime}} \cdot \Delta_{s s s^{\prime \prime} s^{\prime \prime}}-\Delta_{s s s^{\prime} s^{\prime \prime}}^{2}}{\Delta_{s^{\prime} s^{\prime} s^{\prime \prime} s^{\prime \prime}} \cdot \Delta_{s s s^{\prime} s^{\prime \prime}}+\Delta_{s^{\prime \prime} s^{\prime \prime} s s^{\prime} \cdot \Delta_{s^{\prime} s^{\prime} s s^{\prime \prime}}}}
$$

This is found from two relations given by Professor Pearson* by dividing one of them by the other.

(5) Let $\Delta$ be ${ }_{p+1} \stackrel{q}{\Delta}, s^{\prime}=1$ and $s^{\prime \prime}=p+1$, then

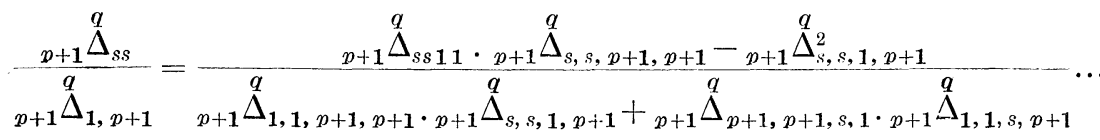

Now

$$
\begin{aligned}
{ }_{p+1}^{q} \Delta_{s s 11} & ={ }_{p}^{q+2} \Delta_{s-1, s-1}, \\
{ }_{p+1} \stackrel{\Delta}{s, s, p+1, p+1}^{q} & ={ }_{p}^{q} \Delta_{s s}, \\
{ }_{p+1} \Delta_{1,1, p+1}, p+1 & =(-1)^{p+1}{ }_{p}^{q+1} \Delta_{1, p}, \\
{ }_{p+1}^{q} \Delta_{s, s, 1, p+1} & =(-1)^{p}{ }_{p}^{q+1} \Delta_{s-1, s}, \\
& * \text { Biometrika, Vol. xI, pp. } 232-3 .
\end{aligned}
$$




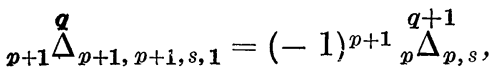

$$
\begin{aligned}
& { }_{p+1} \stackrel{q}{\Delta_{1}, 1, s, p+1}=(-1)^{p+1}{ }_{p}^{q+1} \Delta_{s-1,1},
\end{aligned}
$$

so that all the determinants on the right side in (27) can be evaluated by (25).

They all have the factor

$$
\left\{1^{p-2} \cdot 2^{p-3} \ldots \ldots(p-3)^{2}(p-2)\right\}^{2} \cdot 2^{(p-1)(p-2)}
$$

in common, when that is divided out there remains

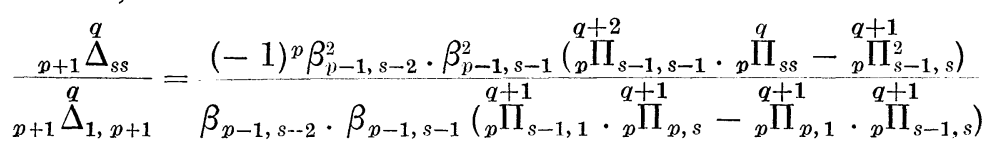

Now indicating by $C_{r}$ the product of the elements of the $r$ th column or $r$ th row in ${ }_{p+1} \stackrel{q}{\Delta}$ and by $e_{r s}$ the element of the ${ }_{p+1} \stackrel{q}{\Delta}$ common for the $r$ th row and $s$ th column we find

$$
\begin{aligned}
{ }_{p+1} \stackrel{q}{\Pi}_{s s} & ={ }_{p}^{q+2} \prod_{s-1, s-1} \cdot \frac{C_{1}^{2}}{e_{11} \cdot e_{s 1}^{2}}, \\
{ }_{p+1} \Pi_{s s}^{q} & ={ }_{p} \prod_{s s}^{q} \cdot \frac{C_{p+1}^{2}}{e_{p+1, p+1} \cdot e_{p+1, s}^{2}}, \\
{ }_{p+1} \prod_{s s}^{q} & ={ }_{p}^{q+1} \prod_{s-1, s} \cdot \frac{C_{1} C_{p+1}}{e_{1, p+1} \cdot e_{s, 1} \cdot e_{s, p+1}} .
\end{aligned}
$$

Hence the factor of the numerator in (28) is reduced to

$$
{ }_{p+1} \prod_{s s}^{q} \frac{e_{s, 1}^{2} \cdot e_{s, p+1}^{2}}{C_{1}^{2} \cdot C_{p+1}^{2}}\left\{e_{11} \cdot e_{p+1, p+1}-e_{1, p+1}^{2}\right\} .
$$

For the $\Pi$ 's of the denominator we find

$$
\begin{aligned}
& { }_{p+1} \prod_{1, p+1}^{q}={ }_{p}^{q+1} \prod_{s-1,1} \cdot \frac{C_{1} \cdot C_{s}}{e_{s 1} \cdot e_{s, p+1} \cdot e_{1,1}}, \\
& { }_{p+1} \stackrel{q}{\Pi}_{1, p+1}={ }_{p}^{q+1} \prod_{p, s} \cdot \frac{C_{p+\mathbf{1}} \cdot C_{s}}{e_{s, p+\mathbf{1}} \cdot e_{p+\mathbf{1}}, p+\mathbf{1}} \cdot e_{\mathbf{1}, s}, \\
& { }_{p+1} \stackrel{q}{\Pi}_{1, p+1}={ }_{p}^{q+1} \prod_{p, 1} \cdot \frac{C_{p+1} \cdot C_{1}}{e_{1, p+1} \cdot e_{11} \cdot e_{p+1}, p+1}, \\
& { }_{p+1}{\stackrel{q}{\prod_{1, p+1}}}^{q}{ }_{p}^{q+1} \Pi_{s-1, s} \cdot \frac{C_{s}^{2}}{e_{s s} \cdot e_{s 1} \cdot e_{s, p+1}},
\end{aligned}
$$

the factor containing $\Pi$ 's of the denominator of $(28)$ is therefore equal to

$$
{ }_{p+1} \prod_{1, p+1}^{q} \frac{e_{1 s} \cdot e_{p+1, s} \cdot e_{1,1} \cdot e_{p+1, p+1}}{C_{1} \cdot C_{p+1} \cdot C_{s}^{2}}\left\{e_{1 s} \cdot e_{p+1, s}-e_{1, p+1} \cdot e_{s s}\right\} .
$$

Introducing these two expressions in (28) and substituting for the one factor $-\frac{p+1}{\prod_{s s}^{q}} \frac{q_{s}}{\prod_{1, p+1}}$ the value $\frac{C_{1} \cdot C_{p+1}}{C_{s}^{2}} \cdot \frac{e_{s s}}{e_{1, p+1}}$ we hence find

$$
\frac{{ }_{p+1} \stackrel{q}{\Delta}_{s s}}{{ }_{p+1} \Delta_{1, p+1}}=(-1)^{p} \beta_{p-1, s-2} \beta_{p-1, s-1} \frac{\frac{1}{e_{1, p+1}^{2}}-\frac{1}{e_{11} \cdot e_{p+1, p+1}}}{\frac{1}{e_{s s} \cdot e_{1, p+1}}-\frac{1}{e_{1, s} \cdot e_{p+1, s}}} \cdot \frac{{ }_{p+1} \prod_{s s}^{q}}{\prod_{1, p+1}}
$$




\section{KIRsTine Smith}

The fraction containing $e$ 's equals

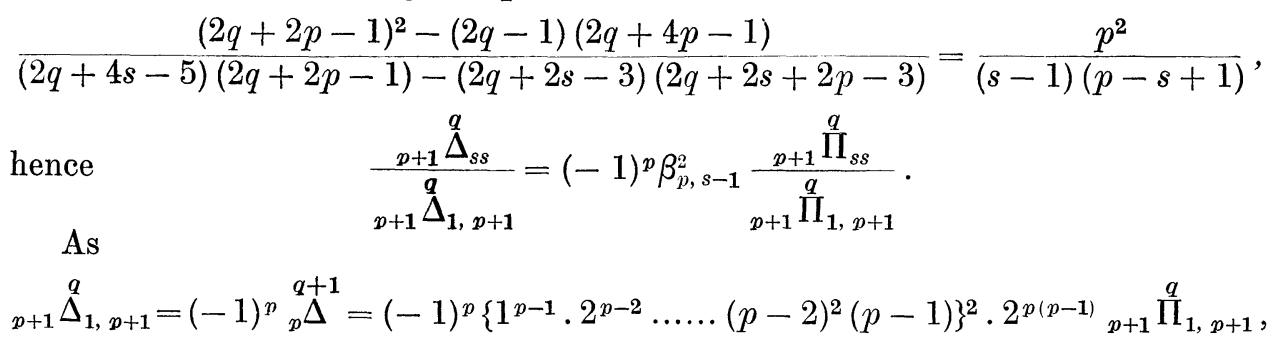

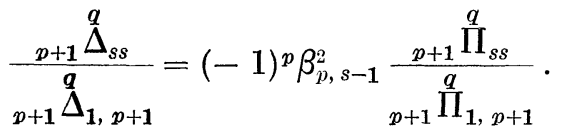
we therefore find

$$
{ }_{p+1} \stackrel{q}{\Delta}_{s s}=\beta_{p, s-1}^{2}\left\{1^{p-1} \cdot 2^{p-2} \ldots \ldots(p-2)^{2}(p-1)\right\}^{2} \cdot 2^{p(p-1)}{ }_{p+1}{\stackrel{q}{\prod_{s s}}},
$$

agreeing with (25).

(6) To evaluate ${ }_{p+1} \stackrel{q}{\Delta} s, p+1$ we shall in (26) put $\Delta={ }_{p+1} \stackrel{q}{\Delta}, s=1, s^{\prime}=s$ and $s^{\prime \prime}=p+1$. Reversing the fractions we then get

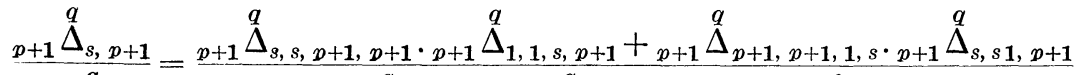

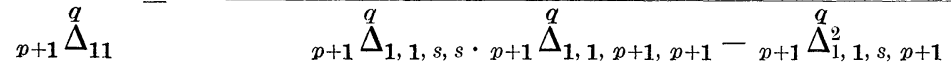

As

$$
\begin{aligned}
& { }_{p+1} \stackrel{q}{\Delta}_{s, s, p+1, p+1}={ }_{p} \stackrel{q}{\Delta}_{s s}, \\
& { }_{p+1} \stackrel{q}{\Delta_{1,1}, s, p+1}={ }_{p}^{q+2} \Delta_{s-1, p}=(-1)^{p+1}{ }_{p}^{q+1} \Delta_{1, s-1}, \\
& { }_{p+1} \stackrel{q}{\Delta}_{p+\mathbf{1}, p+\mathbf{1}, \mathbf{1}, s}=(-1)^{p+1}{ }_{p}^{q+1} \Delta_{s, p}, \\
& { }_{p+1} \stackrel{q}{\Delta} \Delta_{s, s, 1, p+1}=(-1)^{p}{ }_{p}^{q+1} \Delta_{s-1, s}, \\
& { }_{p+1} \stackrel{q}{\Delta}_{\mathbf{1}, \mathbf{1}, s, s}={ }_{p}^{q+2} \Delta_{s-\mathbf{1}, s-\mathbf{1}}, \\
& { }_{p+1} \stackrel{q}{\Delta}{ }_{1,1, p+1}, p+\mathbf{1}={ }_{p} \stackrel{q}{\Delta}{ }_{1,1},
\end{aligned}
$$

the right side of (29) can be evaluated by (25).

We thus get

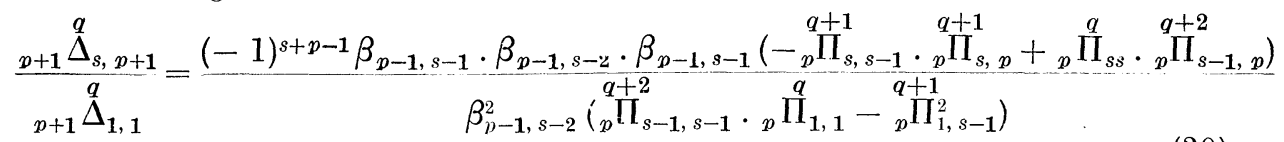

We want here to express the $\Pi$ 's of the numerator by ${ }_{p+1} \stackrel{q}{\Pi}_{s, p+1}$ and those of the denominator by ${ }_{p+1} \stackrel{q}{\prod_{1,1}}$ and we find the following relations

$$
\begin{aligned}
& { }_{p+\mathbf{1}} \stackrel{q}{\Pi}_{s, p+\mathbf{1}}={ }_{p}^{q+1} \prod_{s, s-\mathbf{1}} \cdot \frac{C_{\mathbf{1}} \cdot C_{s}}{e_{\mathbf{1}} \cdot e_{\mathbf{1}, p+1} \cdot e_{s s}}, \\
& { }_{p+1} \stackrel{q}{\Pi}_{s, p+1}={ }_{p}^{q+1} \prod_{s p} \cdot \frac{C_{p+1} \cdot C_{1}}{e_{1, p+1} \cdot e_{p+1}, p+1} \cdot e_{1, s}, \\
& { }_{p+1} \stackrel{q}{\Pi}_{s, p+1}={ }_{p} \prod_{s s} \cdot \frac{C_{s} \cdot C_{p+1}}{e_{s, p+1} \cdot e_{s s} \cdot e_{p+1}, p+1},
\end{aligned}
$$


and

$$
\begin{aligned}
{ }_{p+1} \stackrel{q}{\Pi}_{s, p+1} & ={ }_{p}^{q+2} \prod_{s-1, p} \cdot \frac{C_{1} \cdot C_{1}}{e_{1,1} \cdot e_{1 s} \cdot e_{1, p+1}}, \\
{ }_{p+1} \stackrel{q}{\Pi}_{11} & ={ }_{p}^{q+2} \prod_{s-1, s-1} \cdot \frac{C_{s} \cdot C_{s}}{e_{s s} \cdot e_{1 s}^{2}}, \\
{ }_{p+1} \stackrel{q}{\Pi}_{11} & ={ }_{p} \stackrel{q}{\Pi}_{11} \cdot \frac{C_{p+1}^{2}}{e_{p+1, p+1} \cdot e_{1, p+1}^{2}}, \\
{ }_{p+1} \stackrel{q}{\Pi}_{11} & ={ }_{p}^{q+1} \prod_{1, s-1} \cdot \frac{C_{s} \cdot C_{p+1}}{e_{s, p+1} \cdot e_{1, p+1} \cdot e_{1 s}} .
\end{aligned}
$$

Substituting the $\Pi$ 's found from these relations into (30) and eliminating the

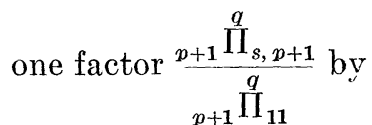

we get

$$
{ }_{p+1} \frac{\stackrel{q}{\Pi}_{s, p+1}^{q}}{\prod_{11}^{q}}=\frac{C_{1}^{2}}{C_{s} \cdot C_{p+1}} \cdot \frac{e_{s, p+1}}{e_{1,1}}
$$

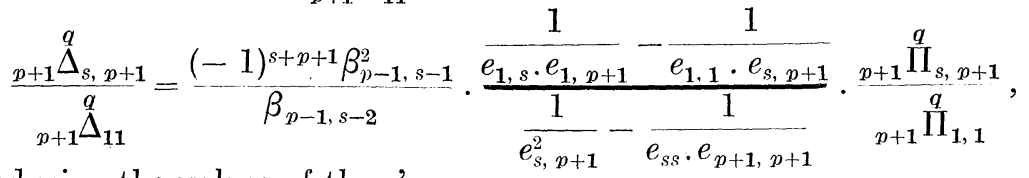

or introducing the values of the $e^{\text {'s }}$

$$
\begin{aligned}
& { }_{p+1} \stackrel{q}{\Delta}_{s, p+1} \\
& { }_{p+1} \stackrel{q}{\Delta}{ }_{1,1} \\
& =\frac{(-1)^{s+p+1} \beta_{p-1, s-1}^{2}}{\beta_{p-1, s-2}} \cdot \frac{(2 q+2 s-3)(2 q+2 p-1)-(2 q-1)(2 q+2 p+2 s-3)}{(2 q+2 p+2 s-3)^{2}-(2 q+4 s-5)(2 q+4 p-1)} \cdot \frac{{ }_{p+1} \prod_{s, p+1}^{q}}{q \stackrel{q}{\Pi}_{1,1}} \\
& =(-1)^{s+p+1} \cdot \beta_{p, s-1} \frac{p+1 \stackrel{q}{\Pi}_{s, p+1}^{q}}{\stackrel{q}{\Pi}_{1,1}} \text {. }
\end{aligned}
$$

$$
{ }_{p+1} \stackrel{q}{\Delta}{ }_{1,1}={ }_{p}^{q+2} \Delta=\left\{1^{p-1} \cdot 2^{p-2} \ldots \ldots(p-2)^{2}(p-1)\right\}^{2} \cdot 2^{p(p-1)}{ }_{p+1} \stackrel{q}{\Pi}_{1,1},
$$

and hence

$$
{ }_{p+1} \stackrel{q}{\Delta}_{s, p+1}=(-1)^{s+p+1} \cdot \beta_{p, s-1}\left\{1^{p-1} \cdot 2^{p-2} \ldots \ldots(p-2)^{2}(p-1)\right\}^{2} \cdot 2^{p(p-1)}{ }_{p+1} \stackrel{q}{\Pi}_{s, p+1}
$$
in agreement with (25).

(7) It now remains to prove that (25) holds for ${ }_{p+1} \stackrel{q}{\Delta} \Delta_{s, r}$ when both $s$ and $r$ are different from 1 and $p+1$, and $r$ different from $s$.

For this shall be used the relation

$$
\Delta . \Delta_{s s s^{\prime} s^{\prime \prime}}=\Delta_{s s} \cdot \Delta_{s^{\prime} s^{\prime \prime}}-\Delta_{s s^{\prime}} \cdot \Delta_{s s^{\prime \prime}}
$$
between an orthosymmetrical determinant and its minors.

Putting $\Delta={ }_{p+1} \Delta, s=p+1, s^{\prime}=r$ and $s^{\prime \prime}=s$ and solving the equation with regard to ${ }_{p+1} \Delta_{r, s}$ we have

where

$$
\begin{gathered}
{ }_{p+\mathbf{1}} \Delta_{r, s}=\frac{1}{{ }_{p+\mathbf{1}} \Delta_{p+\mathbf{1}, p+\mathbf{1}}}\left({ }_{p+\mathbf{1}} \Delta_{p+\mathbf{1}} \Delta_{p+\mathbf{1}, p+\mathbf{1}, r, s} \Delta_{p+\mathbf{1}, p+\mathbf{1}, r, s}+{ }_{p} \Delta_{r, s} .\right. \\
\left.{ }_{p+\mathbf{1}} \Delta_{p+\mathbf{1}, r} \cdot{ }_{p+\mathbf{1}} \Delta_{p+\mathbf{1}, s}\right),
\end{gathered}
$$




\section{KIRSTINE SMITH}

Evaluating this by (24) and (25) we get

$$
\begin{aligned}
& { }_{p+1} \stackrel{q}{\Delta}_{r, s}=\frac{(-1)^{r+s}}{{ }_{p} \prod}\left\{1^{p-1} \cdot 2^{p-2} \ldots \ldots(p-2)^{2}(p-1)\right\}^{2} \cdot 2^{p(p-1)}
\end{aligned}
$$

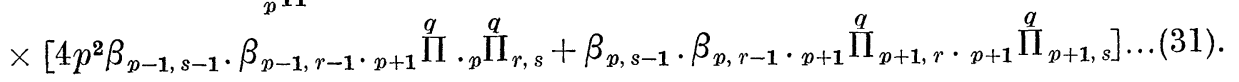

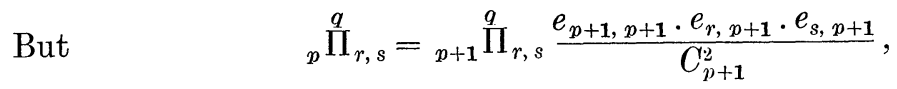

$$
\begin{aligned}
& { }_{p+1} \stackrel{q}{\Pi}_{p+1, r}={ }_{p+1} \stackrel{q}{\Pi}_{r, s} \frac{C_{s}}{C_{p+1}} \cdot \frac{e_{r, p+1}}{e_{r, s}}, \\
& { }_{p+1} \stackrel{q}{\Pi}={ }_{p}^{q} \prod^{\frac{C_{p+1}^{2}}{e_{p+1, p+1}}}, \\
& { }_{p+1} \prod_{p+1, s}={ }_{p} \prod^{q} \frac{C_{p+1}}{C_{s}} \cdot \frac{e_{s, p+1}}{e_{p+1, p+1}} .
\end{aligned}
$$

Substituting these values in (31) we find

$$
\begin{aligned}
& \left.{ }_{p+1} \stackrel{q}{\Delta}_{r, s}=(-1)^{r+s}, 1^{p-1} \cdot 2^{p-2} \ldots(p-2)^{2}(p-1)\right\}^{2} \cdot 2^{p(p-1)} \cdot \beta_{p-1, s-1} \cdot \beta_{p-1, r-1} \cdot p^{2} \cdot p+1 \prod_{r, s}^{q} \\
& \times \frac{\left[4+\frac{1}{(p-s+1)(p-r+1) e_{r, s} \cdot e_{p+1, p+1}}\right]}{\frac{1}{e_{r, p+1}} \cdot \frac{1}{e_{s, p+1}}},
\end{aligned}
$$

and as the last fraction equals

$$
\begin{gathered}
\frac{1}{(p-s+1)(p-r+1)}, \\
{ }_{p+1}^{q} \stackrel{q}{r, s}_{r}=(-1)^{r+s} \beta_{p, s-1} \cdot \beta_{p, r-1}\left\{1^{p-1} \cdot 2^{p-2} \ldots(p-2)^{2}(p-1)\right\}^{2} \cdot 2^{p(p-1)}{ }_{p+1} \prod_{r, s}^{q},
\end{gathered}
$$

with which the proof by induction for (25) is carried through.

(8) We shall now return to (23). It consists of $2 p+1$ terms of which the $(2 r+1)$ st originally was found as $\left({ }_{2 r} \sigma_{y}^{2}-{ }_{2 r-1} \sigma_{\eta !}^{2}\right)$ so that

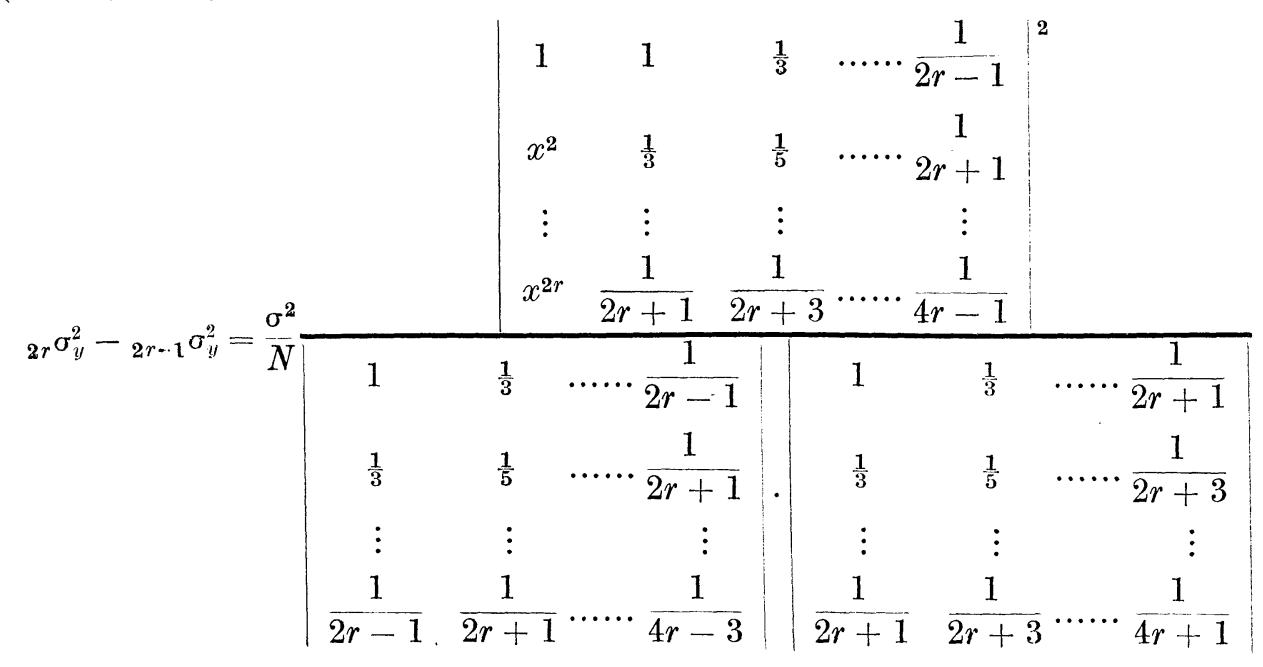




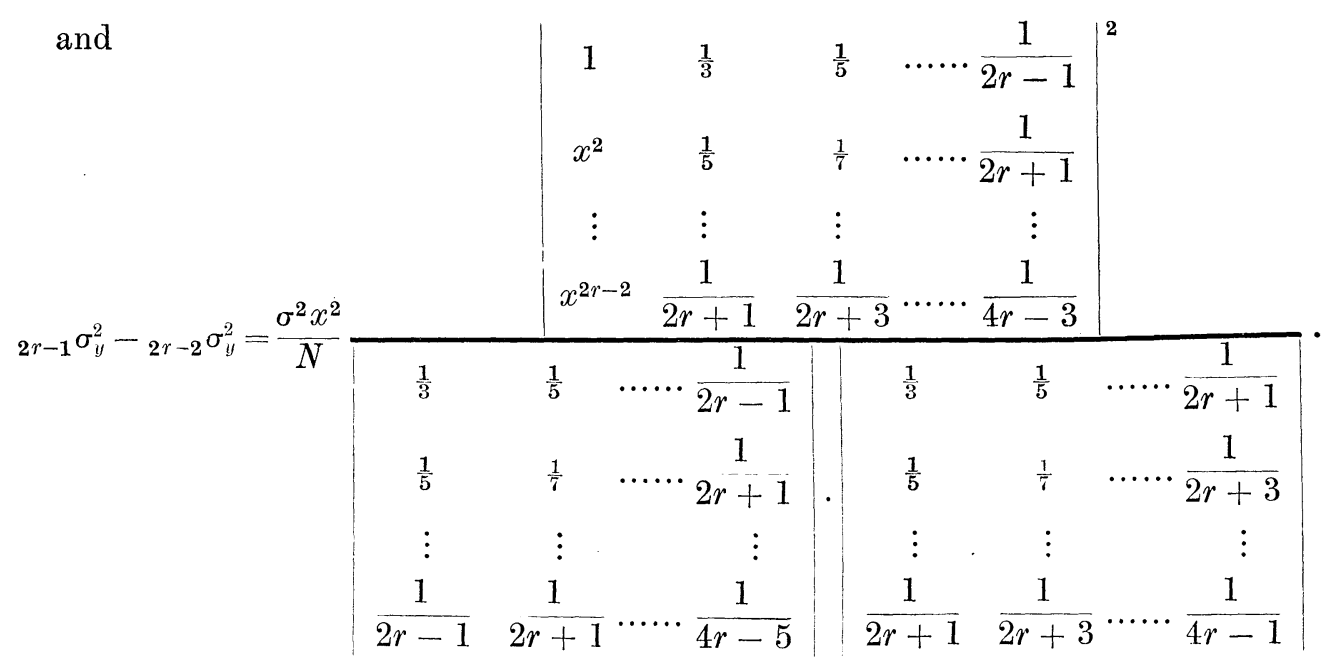

With the notations later adopted we therefore find

and

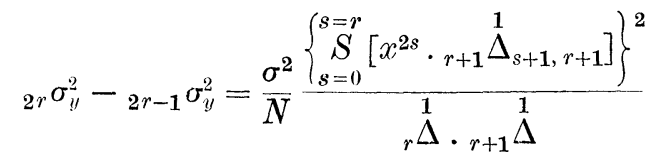

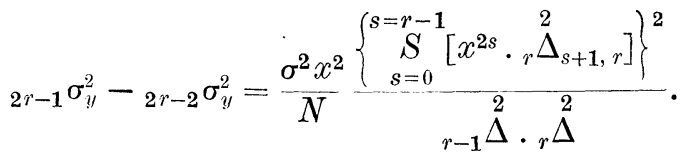

Substituting the values for $\Delta^{\prime}$ s from (24) and (25) we get

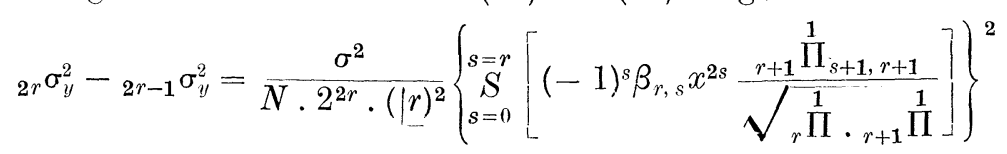

and

$$
{ }_{2 r-1} \sigma_{y}^{2}-{ }_{2 r-2} \sigma_{y}^{2}=\frac{\sigma^{2} x^{2}}{N \cdot 2^{2 r-2} \cdot\left(\underline{\mid r-1)^{2}}\right.}\left\{\underset{s=0}{s=r-1}\left[(-1)^{s} \beta_{r-1, s} x^{2 s} \frac{{ }_{r} \Pi_{s+1, r}}{\sqrt{{ }_{r-1} \Pi^{2} \cdot{ }_{r} \Pi}}\right]\right\}^{2},
$$

or, as

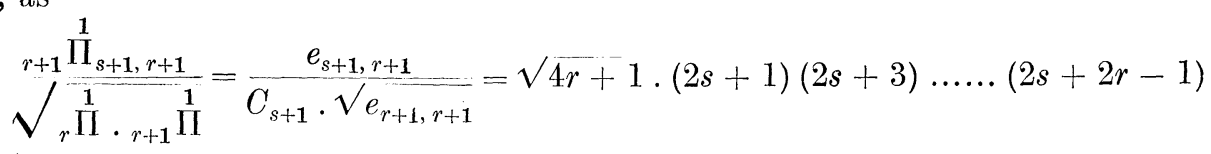
and

$$
\begin{gathered}
\frac{{ }_{r}^{2} \prod_{s+1, r}}{\sqrt{{ }_{r-1} \Pi \Pi_{r} \Pi}}=\frac{e_{s+1, r}}{C_{s+1} \cdot \sqrt{e_{r, r}}}=\sqrt{4 r-1} \cdot(2 s+3)(2 s+5) \ldots \ldots(2 s+2 r-1)^{*}, \\
{ }_{2 r} \sigma_{y}^{2}-{ }_{2 r-1} \sigma_{y}^{2}=\frac{\sigma^{2}(4 r+1)}{N(\mid r)^{2} \cdot 2^{2 r}}\left\{{\left.\underset{s=0}{S=r}\left[(-1)^{s} \beta_{r, s} x^{2 s}(2 s+1)(2 s+3) \ldots \ldots(2 s+2 r-1)\right]\right\}^{2}}^{S},\right.
\end{gathered}
$$

* The $e$ 's and $C$ do not of course have the same value in the two equations as they represent columns and elements in two different determinants. 


\section{KIRSTine SMith}

and

$$
\begin{array}{r}
{ }_{2 r-1} \sigma_{y}^{2}-{ }_{2 r-2} \sigma_{y}^{2}=\frac{\sigma^{2}(4 r-1) x^{2}}{N(\underline{\mid r-1})^{2} \cdot 2^{2 r-2}}\left\{\begin{array} { r } 
{ s = r - 1 } \\
{ s = 0 }
\end{array} \left[(-1)^{s} \beta_{r-1, s} x^{2 s}(2 s+3)(2 s+5) \ldots \ldots .\right.\right. \\
(2 s+2 r-1)]\}^{2} \ldots \ldots \ldots \ldots(33),
\end{array}
$$

which enables us to form ${ }_{n} \sigma_{y}^{2}$ by successive summations from ${ }_{0} \sigma_{y}^{2}=\frac{\sigma^{2}}{N}$.

Before investigating the curve for ${ }_{n} \sigma_{y}^{2}$ for a special $n$ we shall first look at ${ }_{n} \sigma^{2}$ for $x=0$ and $x= \pm 1$.

(9) From (33) we see that when $x=0$

$$
{ }_{2 r-1} \sigma_{y}^{2}={ }_{2 r-2} \sigma_{y}^{2},
$$

${ }_{2 p} \sigma_{y}^{2}$ is for $x=0$ most easily evaluated from the formula (13).

Remembering that in our case $m_{2 r}=\frac{1}{2 r+1}$ we find from this

and hence by (24) and (25)

$$
\underset{2 p}{x=0} \sigma_{y}^{2}=\frac{\sigma^{2}}{\bar{N}} \frac{{ }_{p+1}^{1} \Delta_{1,1}}{{ }_{p+1} \stackrel{\Delta}{\Delta}},
$$

$$
\begin{gathered}
x=0 \\
2 p+1 \\
\sigma_{y}^{2}
\end{gathered}=\underset{2 p}{x=0} \sigma_{y}^{2}=\frac{\sigma^{2}}{N}\left\{\frac{3}{2} \cdot \frac{5}{4} \cdot \frac{7}{6} \ldots \ldots \frac{2 p+1}{2 p}\right\}^{2}
$$

(10) To evaluate ${ }_{n} \sigma_{y}^{2}$ for $x= \pm 1$ we use (32) and (33). The sum in (32) may be considered as

$$
S_{2 r}=\frac{d}{d x} \frac{1}{x} \frac{d}{d x} \ldots \ldots \frac{1}{x} \frac{d\left\{x^{2 r-1}\left(x^{2}-1\right)^{r}\right\}}{d x}
$$

with a number $r$ of differentiations. If these operations are undertaken directly upon $x^{2 r-1}\left(x^{2}-1\right)^{r}$ the result is

of which only

$$
\alpha_{r}\left(x^{2}-1\right)^{r}+\alpha_{r-1}\left(x^{2}-1\right)^{r-1}+\ldots \ldots \alpha_{1}\left(x^{2}-1\right)+\alpha_{0},
$$

remains for $x= \pm 1$.

$$
a_{0}=2 r(2 r-2) \ldots \ldots .4 .2=\underline{r} .2^{r}
$$

Corresponding to this the sum in (33) comes out from

$$
S_{2 r-1}=\frac{d}{d x} \frac{1}{x} \frac{d}{d x} \ldots \cdots \frac{d}{d x} \frac{1}{x} \frac{d\left\{x^{2 r-1}\left(x^{2}-1\right)^{r-1}\right\}}{d x}
$$

by taking $(r-1)$ differentiations and therefore

$$
S_{2 r-1} \text { equals, for } x= \pm 1, \quad(2 r-2)(2 r-4) \ldots \ldots 4.2=\mid r-1.2^{r-1} .
$$

Hence

$$
\begin{aligned}
& x^{2}=1 \\
& { }_{2 r} \sigma_{y}^{2}
\end{aligned}-{ }_{2 r-1}^{x^{2}=1} \sigma_{y}^{2}=\frac{\sigma^{2}}{N}(4 r+1)
$$

and

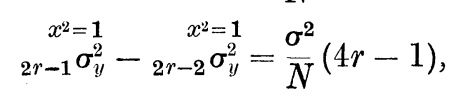

or since

$$
{ }_{0} \sigma_{y}^{2}=\frac{\sigma^{2}}{N},
$$

$$
\begin{gathered}
\begin{array}{c}
x^{2}=1 \\
\sigma_{y}^{2}
\end{array}=\frac{\sigma^{2}}{N}\{1+3+5+\ldots \ldots(2 n+1)\}, \\
x^{2}=1 \\
\sigma_{y}^{2}=\frac{\sigma^{2}}{N}(n+1)^{2} \ldots \ldots \ldots \ldots \ldots \ldots \ldots \ldots \ldots \ldots \ldots \ldots \ldots \ldots \ldots \ldots \ldots \ldots
\end{gathered}
$$




\section{Choice in the Distribution of Observations}

(11) In Section I under (7) it was found that

$$
\int \frac{\psi(x)}{f(x)}{ }_{n} \sigma_{y}^{2} d x=\sigma^{2}(n+1)
$$

when the integration was taken over the places of observation. For the present distribution $f(x)$ is $1, \psi(x)$ constant and $\int \psi(x) d x=N$, hence the mean of ${ }_{n} \sigma_{y}^{2}$ in the range of observations is for a uniform continuous distribution

$$
\frac{\sigma^{2}}{N}(n+1) \text {. }
$$

For the grouped observations in Section II we find by integration of the formulae for functions from the first to the sixth degree that

$$
\frac{1}{2} \int_{-1}^{1}{ }_{n} \sigma_{y}^{2} d x=\frac{\sigma^{2}}{N}(n+1)\left(1-\frac{1}{2 n+1}\right) .
$$

IV. Uniform continuous distribution of observations with constant standard deviation. Special formulae.

(1) Let ${ }_{n} \sigma_{y}^{2}-{ }_{n-1} \sigma_{y}^{2}$ be indicated by $S_{n}$, then the formulae (32) and (33) give us

$$
\left.\begin{array}{l}
S_{1}=\frac{\sigma^{2}}{N} \cdot 3 x^{2} \\
S_{2}=\frac{\sigma^{2}}{N} \cdot \frac{5}{4}\left(1-3 x^{2}\right)^{2} \\
S_{3}=\frac{\sigma^{2}}{N} \cdot \frac{7}{4} x^{2}\left(3-5 x^{2}\right)^{2} \\
S_{4}=\frac{\sigma^{2}}{N} \cdot \frac{9}{61}\left(3-30 x^{2}+35 x^{4}\right)^{2} \\
S_{5}=\frac{\sigma^{2}}{N} \cdot \frac{11 x^{2}}{64}\left(15-70 x^{2}+63 x^{4}\right)^{2} \\
S_{6}=\frac{\sigma^{2}}{N} \cdot \frac{13}{9 \times 256}\left(15-315 x^{2}+945 x^{4}-693 x^{6}\right)^{2}
\end{array}\right\}
$$

from which we form ${ }_{n} \sigma_{y}^{2}$ beginning with

$$
\begin{aligned}
{ }_{0} \sigma_{y}^{2} & =\frac{\sigma^{2}}{N} \\
{ }_{1} \sigma_{y,}^{2} & =\frac{\sigma^{2}}{N}\left(1+3 x^{2}\right) \\
{ }_{2} \sigma_{y \prime}^{2} & =\frac{\sigma^{2}}{N}\left(1+3 x^{2}+\frac{\tilde{5}}{4}\left(1-3 x^{2}\right)^{2}\right)=\frac{\sigma^{2}}{N} \cdot \frac{9}{4}\left(1-2 x^{2}+5 x^{4}\right),
\end{aligned}
$$

and further in the same way

$$
\left.\begin{array}{rl}
{ }_{3} \sigma_{y !}^{2} & =\frac{\sigma^{2}}{N} \cdot \frac{1}{4}\left(9+45 x^{2}-165 x^{4}+175 x^{6}\right) \\
{ }_{4} \sigma_{y}^{2} & =\frac{\sigma^{2}}{N} \cdot \frac{25}{64}\left(9-36 x^{2}+294 x^{4}-644 x^{6}+441 x^{8}\right) \\
{ }_{5} \sigma_{y}^{2} & =\frac{\sigma^{2}}{N} \cdot \frac{9}{64}\left(25+175 x^{2}-1750 x^{4}+6510 x^{6}-9555 x^{8}+4851 x^{10}\right) \\
{ }_{6} \sigma_{y}^{2} & =\frac{\sigma^{2}}{N} \cdot \frac{7}{256}\left(175-1050 x^{2}+17325 x^{4}-93660 x^{6}+225225 x^{8}+\right. \\
\left.-245322 x^{10}+99099 x^{12}\right)
\end{array}\right)
$$




\section{Kirstine Smiti}

(2) Since ${ }_{n} \sigma_{y}^{2}={ }_{n-1} \sigma_{y}^{2}+S_{n}$ the curve for ${ }_{n} \sigma_{y}^{2}$ is entirely above the ${ }_{n-1} \sigma_{y}^{2}$ curve except where $S_{n}=0$.

Solving the equations $S_{n}=0$ the following roots are found:

$$
\begin{aligned}
& \text { For } S_{1}=0 \quad x=0 \\
& \text {, } S_{2}=0 \quad x= \pm \sqrt{\frac{1}{3}}= \pm \cdot 5773 \\
& \text { " } S_{3}=0 \quad x=0 \quad x= \pm \sqrt{\frac{3}{5}}= \pm \cdot 7746 \\
& \text {, } S_{4}=0 \quad x= \pm \sqrt{\frac{15 \pm 2 \sqrt{30}}{35}}= \pm\left\{\begin{array}{l}
\cdot 8611 \\
\cdot 3400
\end{array}\right. \\
& \text {, } S_{5}=0 \quad x=0 \quad x= \pm \sqrt{\frac{35 \pm 2 \sqrt{70}}{63}}= \pm\left\{\begin{array}{l}
\cdot 9030 \\
\cdot 5438
\end{array}\right. \\
& \text {, } S_{6}=0 \quad x= \pm\left\{\begin{array}{l}
\cdot 2386 \\
\cdot 6612 \\
\cdot 9325
\end{array}\right.
\end{aligned}
$$

Since all the roots are rational and all lie between -1 and $+1,{ }_{n} \sigma_{y}^{2}$ therefore equals ${ }_{n-1} \sigma_{y}^{2}$ for $n$ values of $x$ all of which are inside the range of the observations.

The adjusted values of the functions at these abscissae appear to be of special interest since they are uncorrelated as was shown in Section I under (9).

(3) Looking at Diagram 2, representing the curves of ${ }_{n} \sigma_{y}$ up to $n=6$, it is seen, as was also clear from the formula for $\begin{gathered}x=0 \\ \sigma_{y}^{2}\end{gathered}$ and $\begin{gathered}x^{2}=1 \\ \sigma_{y}^{2}\end{gathered}$ given in the last section, that while the standard deviation in the middle of the range increases slowly with the degree of function it increases very rapidly at the ends of the range. At $x=0$ the curve has a minimum when the degree of function is odd and a maximum when it is even. Besides that the curve has $(2 n-2)$ maxima and minima between -1 and 1 . As the curve for ${ }_{n} \sigma_{y}^{2}$ is of the $2 n$th degree, ${ }_{n} \sigma_{y}^{2}$ is therefore increasing for $x$ increasing above 1 or for $x$ decreasing below -1 .

The abscissae of the maxima and minima are given in the following table.

$$
\begin{aligned}
& \text { Degree of } \\
& \text { function } \\
& 1 \\
& \text { Abscissae of maxima } \\
& 2+0 \\
& 3 \\
& \pm \sqrt{\frac{1}{5}}= \pm \cdot 4472 \\
& \pm \cdot 4472 \\
& \left\{\begin{array}{c}
0 \\
\pm \sqrt{\frac{3}{7}}= \pm \cdot 6547
\end{array}\right. \\
& 5 \quad \pm \sqrt{\frac{7 \pm 2 \sqrt{7}}{21}}= \pm\left\{\begin{array}{l}
\cdot 7651 \\
\cdot 2852
\end{array}\right. \\
& 6\left\{\begin{array}{l}
\frac{0}{15 \pm 2 \sqrt{15}} \\
33
\end{array}= \pm\left\{\begin{array}{l}
\cdot 8302 \\
\cdot 4689
\end{array}\right.\right. \\
& \begin{array}{c}
\begin{array}{c}
\text { Abscissae of minima } \\
0
\end{array} \\
\pm \sqrt{\frac{1}{5}}= \pm \cdot 4472 \\
\left\{\begin{array}{l}
0 \\
\pm \sqrt{\frac{3}{7}}= \pm \cdot 6547
\end{array}\right. \\
\pm \sqrt{\frac{7 \pm 2 \sqrt{7}}{21}}= \pm\left\{\begin{array}{l}
\cdot 7651 \\
\cdot 2852
\end{array}\right. \\
\left\{\sqrt{\frac{15 \pm 2 \sqrt{15}}{33}}= \pm\left\{\begin{array}{l}
\cdot 8302 \\
\cdot 4689
\end{array}\right.\right. \\
\pm\left\{\begin{array}{l}
\cdot 8718 \\
\cdot 5917 \\
\cdot 2093
\end{array}\right.
\end{array}
\end{aligned}
$$




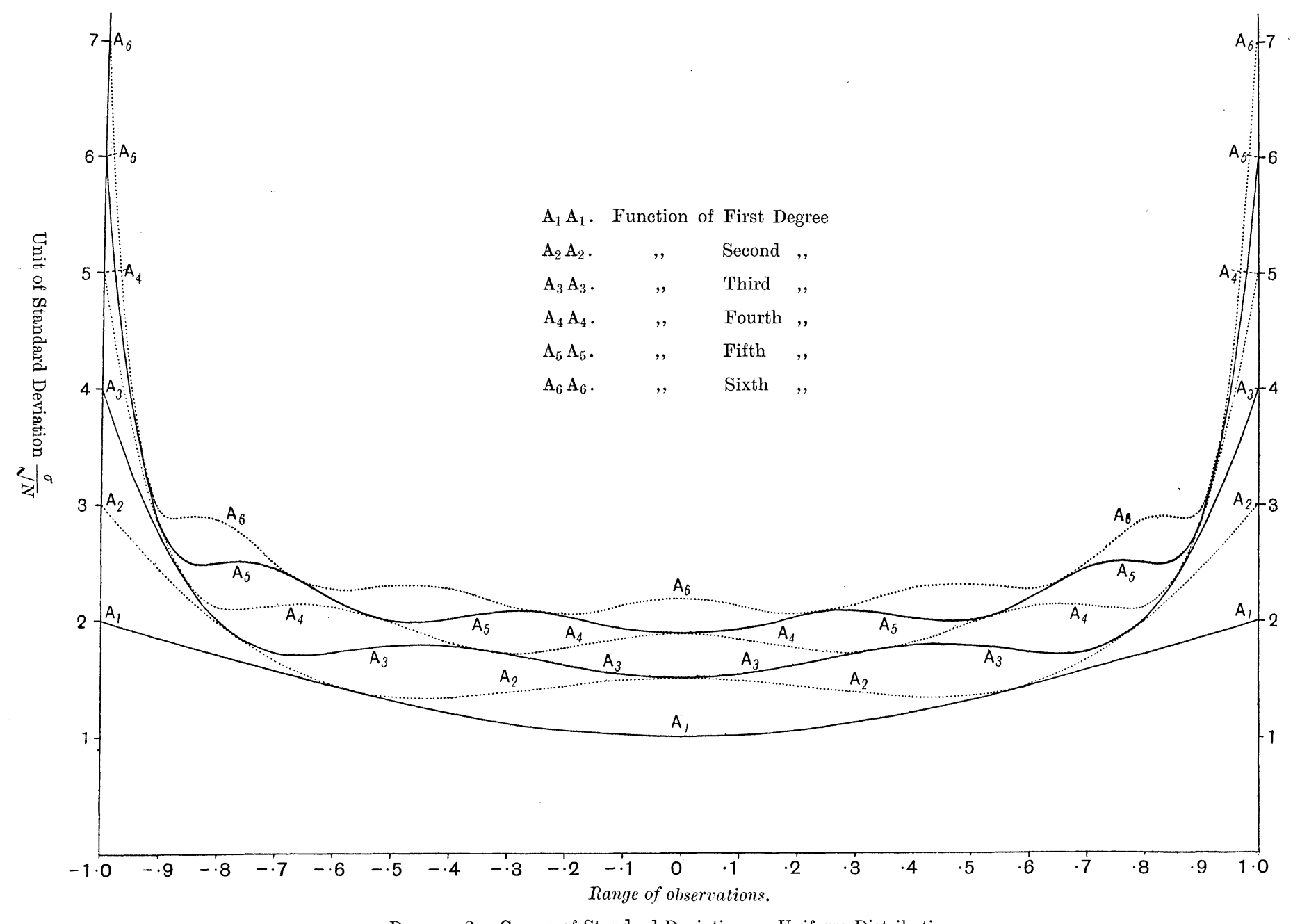

Diagram 2. Curves of Standard Deviations. Uniform Distribution. 
Hence the curve for ${ }_{2 p+1} \sigma_{y}^{2}$ has a maximum for the abscissae at which ${ }_{2 p} \sigma_{y}^{2}$ has a minimum. A comparison with the results in Section II shows that the abscissae of the maxima found here are the same as those of the best places of observation for $(n+1)$ equally big groups of observations of a function of the $n$th degree. These places tally with the places where ${ }_{n} \sigma_{y}^{2}$ was a maximum. Thus if we imagine that we had started the investigations with a uniform distribution of observations, and to lower the maxima of the curve of standard deviation had put clusters of observations at those maxima and at the ends of the range we should not get the best curve of standard deviation till all the observations of the continuous distribution had been distributed at the $n-1$ places of maxima and at 1 and -1 .

The minima of the standard deviations obtained from a uniform continuous distribution and the $(n+1)$ best groups of observations do not fall at the same abscissae.

(4) The curves are very far from our ideal of a constant standard deviation throughout the range. To obtain the same maximum of standard deviation as $(n+1)$ groups could give us we should have to limit the part of the range used to the following fractions of the range:

$\begin{array}{cc}\text { for 1st degree } & \cdot 58 \\ \text { " 2nd " } & .73 \\ \text { " 3rd " } & .80 \\ \text { " 4th " } & .84 \\ \text { " 5th " } & .83 \\ \text { "6th " } & .73\end{array}$

It is not likely that the range of values of the function which we investigate would only be of interest inside a range so much smaller than that within which we might actually observe; further it seems likely that observations all of which were taken inside the smaller part of the range would give better information for that special interval. I shall therefore examine in the following sections if a uniform distribution of observations to which is added clusters of observations at the ends of the range will not possibly give a more satisfactory curve of standard deviations.

V. Uniform continuous distribution of observations with additional observations clustered at the ends of the range; constant standard deviation of observations. General formulae.

(1) Suppose we have $N \cdot \frac{1}{1+a}$ observations uniformly distributed from -1 to 1 and besides $\frac{N}{2} \cdot \frac{a}{1+\alpha}$ observations at -1 and the same number at 1 . We then have

$$
\mu_{2 r}=\frac{1}{N}\left\{\int_{-1}^{1} \frac{N x^{2 r}}{2(1+\alpha)} d x+\frac{N \alpha}{1+\alpha}\right\}
$$


or

$$
\mu_{2 r}=\frac{1}{1+\alpha}\left(\frac{1}{2 r+1}+\alpha\right)
$$

and

$$
\mu_{2 r+1}=0 \text {. }
$$

According to (13) and (14) we find,

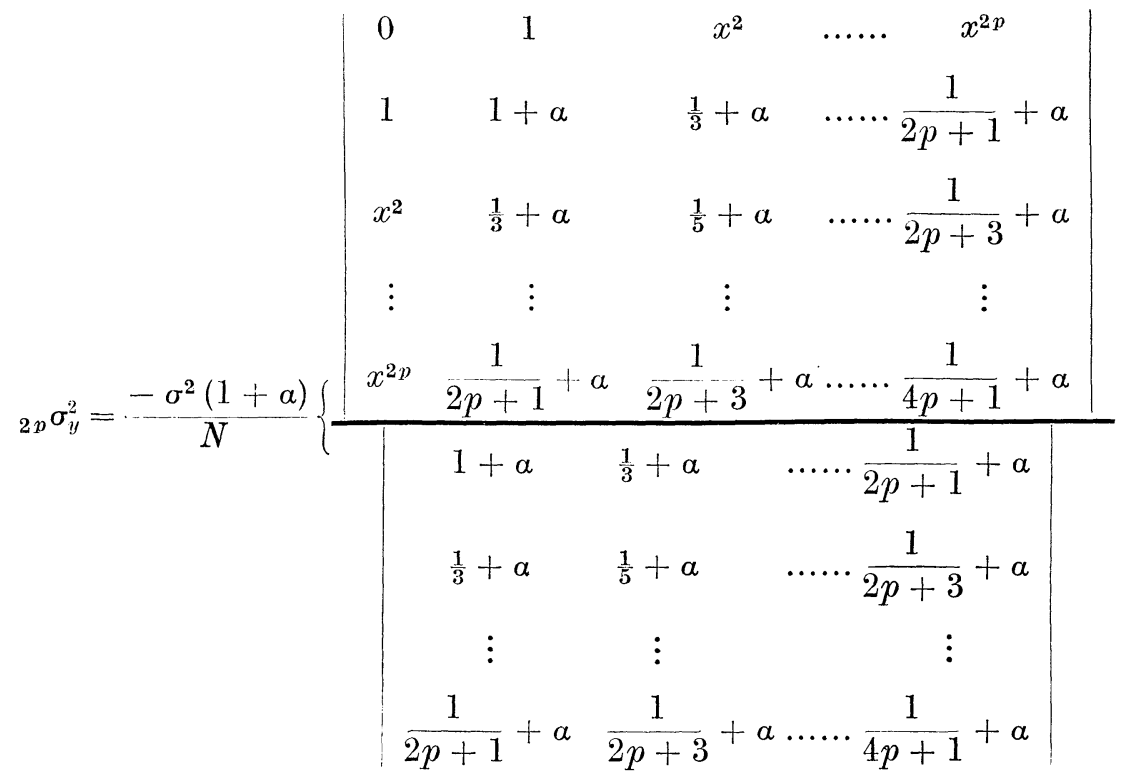

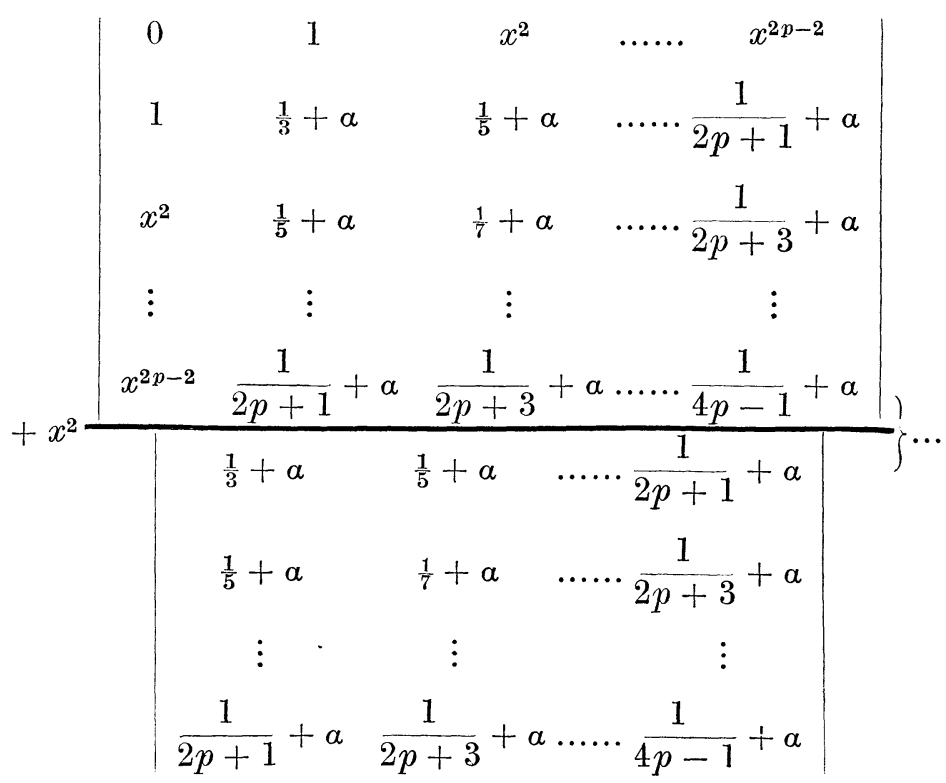


Kirstine Sмith

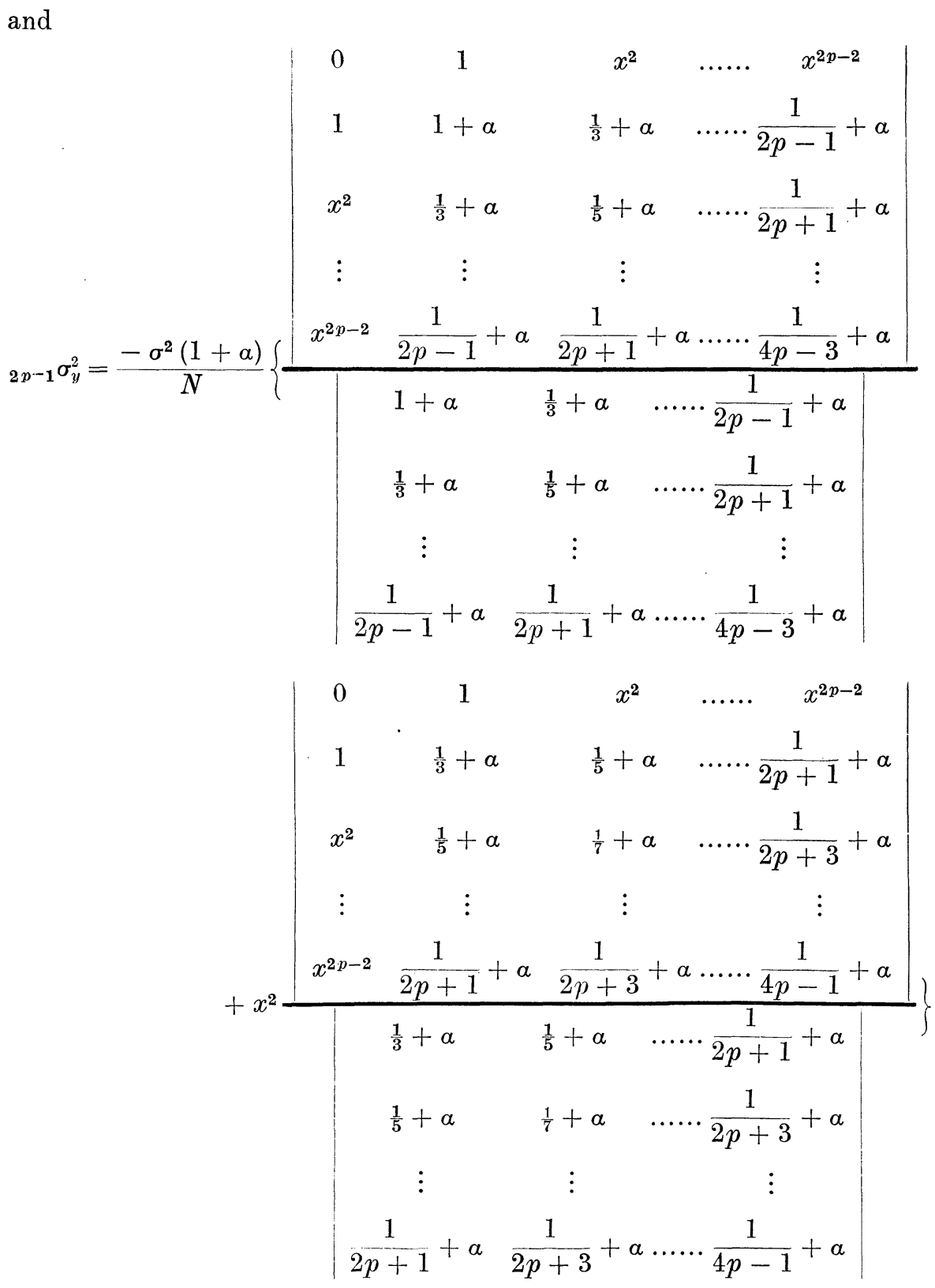


and, according to I under (6),

$$
\begin{aligned}
& { }_{2 p} \sigma_{y}^{z}-{ }_{2 p-1} \sigma_{y}^{2}=\frac{\sigma^{2}}{N}(1+\alpha) \times
\end{aligned}
$$

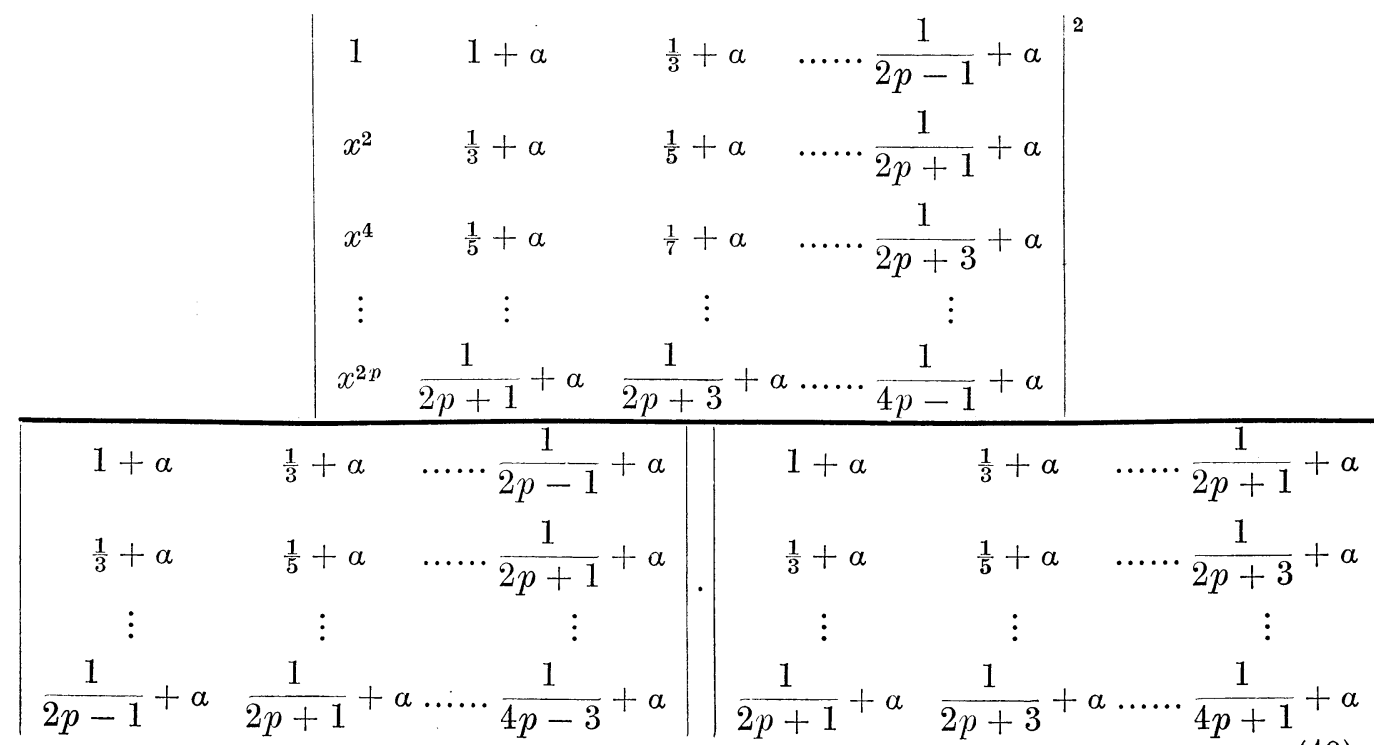

and

$$
{ }_{2 p+1} \sigma^{2}-{ }_{2 p} \sigma_{y}^{2}=\frac{\sigma^{2}(1+\alpha)}{N} x^{2} \times
$$

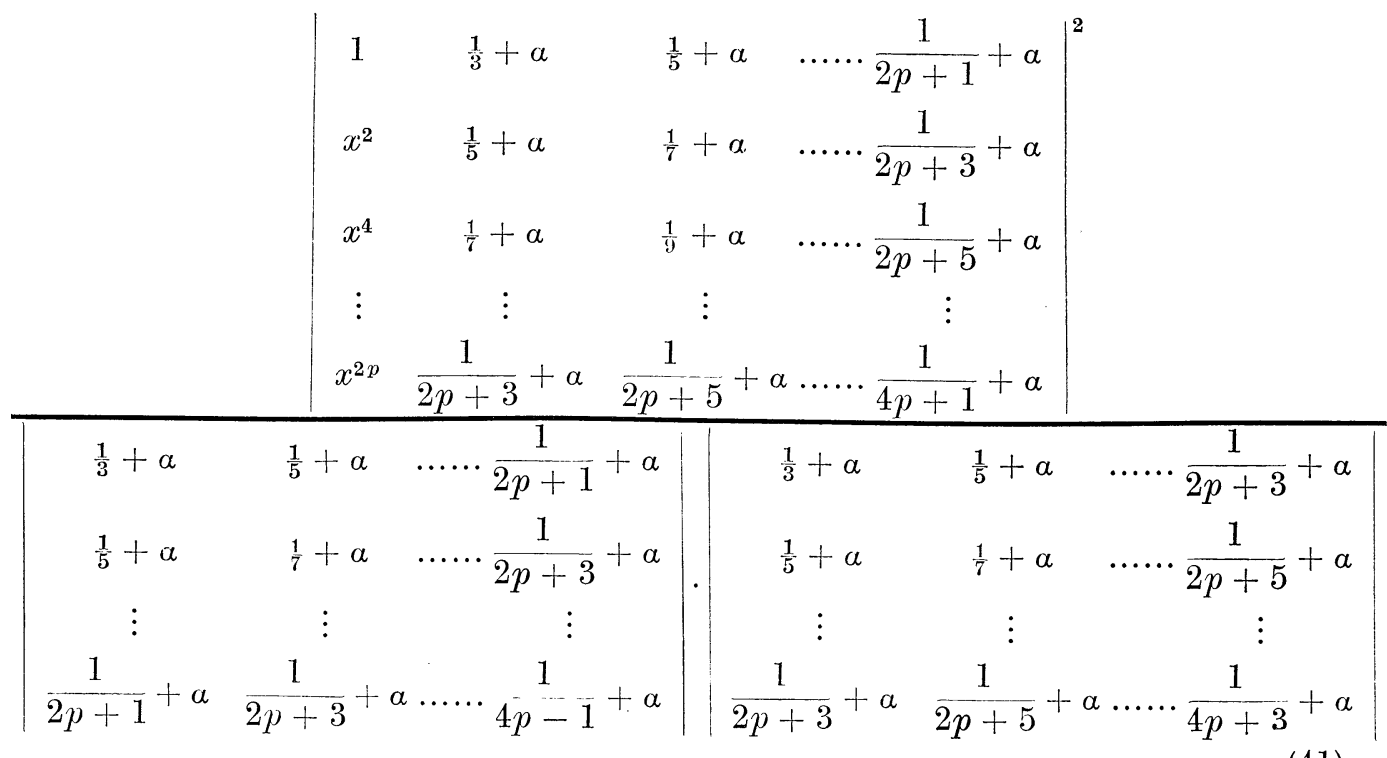

(2) For the reduction of these formulae we have to evaluate the determinant, of $p$ th order 


\section{Kirstine Suith}

$$
\begin{aligned}
& \frac{1}{2 q-1}+\alpha \quad \frac{1}{2 q+1}+\alpha \quad \cdots \cdots \frac{1}{2 q+2 p-3}+\alpha \\
& { }_{p}^{q} \delta=\begin{array}{ccc}
\frac{1}{2 q+1}+\alpha & \frac{1}{2 q+3}+\alpha & \cdots \cdots \frac{1}{2 q+2 p-1}+\alpha \\
\vdots & \vdots & \vdots
\end{array} . \\
& \frac{1}{2 q+2 p-3}+a \frac{1}{2 q+2 p-1}+\alpha \cdots \cdots \frac{1}{2 q+4 p-5}+\alpha
\end{aligned}
$$

By subtracting from the elements of each row the elements of the proceeding and leaving the first row as it is, it is transformed to ${ }_{p}^{q} \delta=(-1)^{p-1} \times$

$$
\left|\begin{array}{cccc}
\frac{1}{2 q-1}+\alpha & \frac{1}{2 q+1}+\alpha & \cdots \cdots & \frac{1}{2 q+2 p-3}+a \\
\frac{2}{(2 q-1)(2 q+1)} & \frac{2}{(2 q+1)(2 q+3)} & \cdots \cdots & \frac{2}{(2 q+2 p-3)(2 q+2 p-1)} \\
\vdots & \vdots & \vdots \\
\frac{2}{(2 q+2 p-5)(2 q+2 p-3)} & (2 q+2 p-3)(2 q+2 p-1) & \cdots \cdots & 2 \\
\hline(2 q+4 p-7)(2 q+4 p-5)
\end{array}\right|,
$$

which when the columns undergo the same process takes the form

$$
\begin{aligned}
& { }_{p}^{\stackrel{q}{\delta}}= \\
& \frac{1}{2 q-1}+\alpha \\
& \frac{2}{(2 q-1)(2 q+1)} \\
& \frac{2}{(2 q+1)(2 q+3)} \\
& \cdots \frac{2}{(2 q+2 p-5)(2 q+2 p-3)} \\
& \frac{2}{(2 q-1)(2 q+1)} \\
& \text { 2. } 4 \\
& 2.4 \\
& 2.4 \\
& 2 \\
& \begin{array}{cc}
(2 q-1)(2 q+1)(2 q+3) & (2 q+1)(2 q+3) \\
2.4 & 2.4
\end{array} \\
& 2.4 \quad 2.4 \\
& (2 q+1)(2 q+3) \\
& (2 q+1)(2 q+3)(2 q+5) \\
& \begin{array}{r}
(2 q+3)(2 q+ \\
\vdots \\
2.4
\end{array} \\
& \text { ! } \\
& \text { 5) }(2 q+7) \\
& \cdot \overline{(2 q+2 p-3)} \\
& \frac{2}{(2 q+2 p-5)(2 q+2 p-3)} \overline{(2 q+2 p-5) \ldots(2 q+2 p-1)} \overline{(2 q+2 p-3) \ldots(2 q+2 p+1)} \cdots \frac{2.4}{(2 q+4 p-9) \ldots(2 q-4 p-5)}
\end{aligned}
$$

Let us introduce the notation

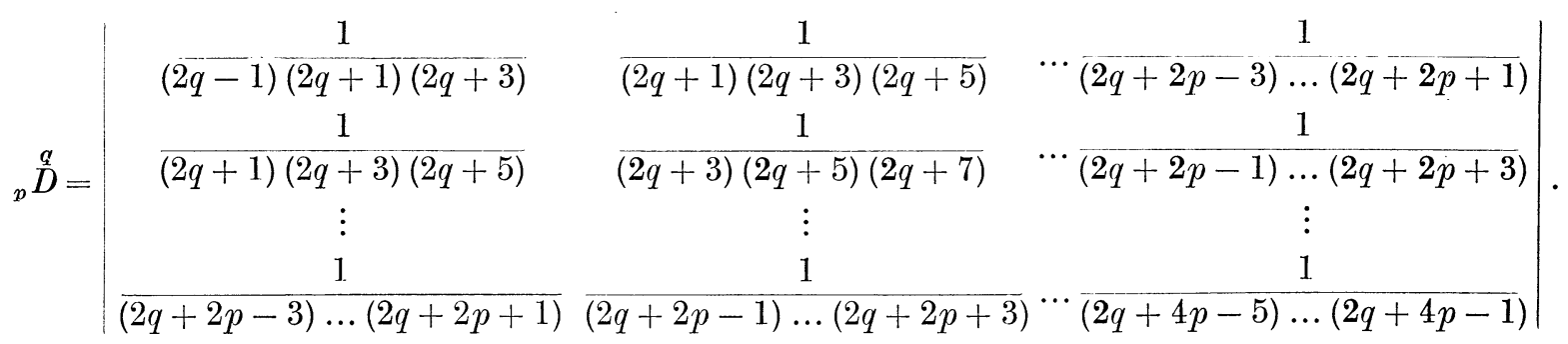

Then, since for $\alpha=0{ }_{p}{ }^{q}$ equals the determinant ${ }_{p} \stackrel{q}{\Delta}$, we have

$$
\stackrel{q}{\delta}={ }_{p} \stackrel{q}{\Delta}+\alpha \cdot 2^{3(p-1)} \cdot{ }_{p-1} \stackrel{q}{D}
$$

and the problem is reduced to the evaluation of ${ }_{p} \stackrel{q}{D}$. 
(3) It shall be proved by induction that

${ }_{p} \stackrel{q}{D}$

$=\frac{\left\{1^{p} \cdot 2^{p-1} \ldots(p-1)^{2} \cdot p\right\}^{2} \cdot 2^{p(p-2)}(p+1)}{(2 q-1)(2 q+1)^{2}(2 q+3)^{3} \ldots(2 q+2 p-5)^{p-1}(2 q+2 p-3)^{p}(2 q+2 p-1)^{p}(2 q+2 p+1)^{p}(2 q+2 p+3)^{p-1} \ldots(2 q+4 p-3)^{2}(2 q+4 p-1)}$

It contains the $2 p+1$ different factors of the elements with indices increasing from 1 at the extreme to $p$ in the middle so that the three factors of which the one diagonal line of the determinant consists occur with the index $p$.

For $p=1$ the formula gives

as it ought to.

$$
{ }_{1}^{q} D=\frac{1}{(2 q-1)(2 q+1)(2 q+3)}
$$

As the determinant is orthosymmetrical the relation

$$
\Delta=\frac{\Delta_{s s} \cdot \Delta_{s^{\prime} s^{\prime}}-\Delta_{s s^{\prime}}^{2}}{\Delta_{s s s^{\prime} s^{\prime}}} \text { holds. }
$$

Applied on ${ }_{p+1} \stackrel{q}{D}$ for $s=1$ and $s^{\prime}=p+1$ it may be written

$$
{ }_{p+1} \stackrel{q}{D}=\frac{{ }_{p}^{q} D \cdot{ }_{p}^{q+2} D-{ }_{p}^{q+1} D^{2}}{{ }_{p-1}^{q+2}}
$$

Looking first at the numerator of (43) we see that it has the same value for the two terms of the numerator of (44), and divided by the corresponding factor of ${ }_{p-1}^{q+2} D$ it becomes

$$
\begin{aligned}
\left\{\frac{1^{2 p} \cdot 2^{2(p-1)} \cdots \cdots(p-2)^{6}(p-1)^{4} p^{2}}{1^{p-1} \cdot 2^{p-2} \cdots \cdots(p-2)^{2}(p-1)}\right\}^{2} & \frac{(p+1)^{2}}{p} \cdot 2^{2 p(p-2)-(p-1)(p-3)} \\
& =\left\{1^{p+1} \cdot 2^{p} \ldots \ldots(p-2)^{4}(p-1)^{3} p^{2}(p+1)\right\}^{2} \frac{1}{p} \cdot 2^{p^{2}-3}
\end{aligned}
$$

To evaluate the factor in ${ }_{p+1} \stackrel{q}{D}$ arising from the denominator of (43) we shall give a table of the indices with which the different factors occur in the $D$ 's and their ratios.

$$
\begin{aligned}
& 2 q-12 q+12 q+3 \ldots 2 q+2 p-5 \quad 2 q+2 p-3 \quad 2 q+2 p-12 q+2 p+1 \quad 2 q+2 p+3 \quad 2 q+2 p+5 \quad 2 q+2 p+7 \quad \ldots 2 q+4 p-12 q+4 p+1 \quad 2 q+4 p+3 \\
& \begin{array}{lllllllllllllll}
{ }_{p}^{q} D & 1 & 2 & 3 & \cdots & p-1 & p & p & p & p-1 & p-2 & p-3 & \cdots & 1 & -
\end{array}
\end{aligned}
$$

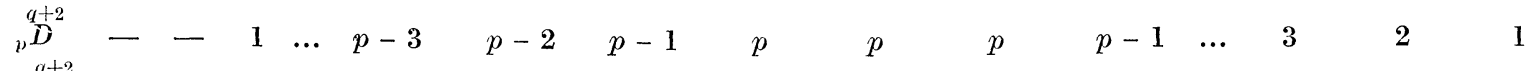

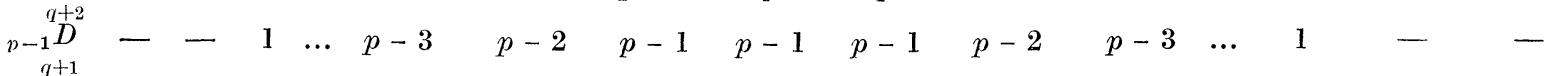

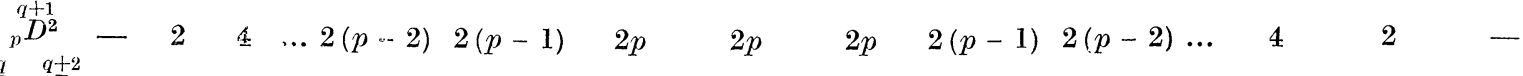

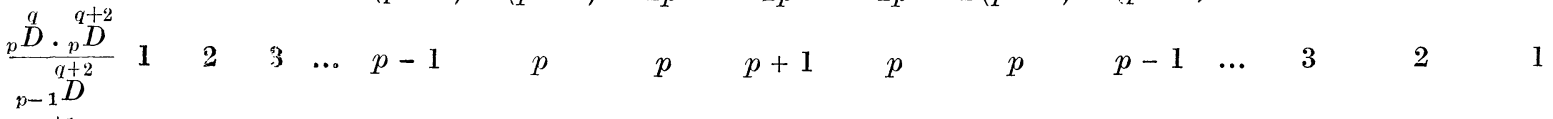

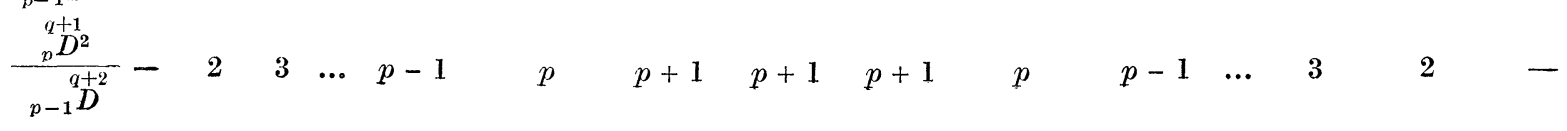


Kirstine SMith

Hence the factor arising from the denominator of $(43)$ is

$$
\begin{aligned}
& \frac{(2 q+2 p-1)(2 q+2 p+3)-(2 q-1)(2 q+4 p+3)}{(2 q-1)(2 q+1)^{2} \ldots(2 q+2 p-3)^{p}(2 q+2 p-1)^{p+1}(2 q+2 p+1)^{p+1}(2 q+2 p+3)^{p+1}(2 q+2 p+5)^{p} \ldots(2 q+4 p+1)^{2}(2 q+4 p+3)} \\
& \text { The numerator of this equals } 4 p(p+2), \\
& \text { multiplying with the factor previously found we therefore get } \\
& =\frac{\left\{1^{p+1} \cdot 2^{p} \ldots(p-1)^{3} p^{2}(p+1)\right\}^{2} \cdot 2^{(p+1)(p-1)} \cdot(p+2)}{(2 q-1)(2 q+1)^{2} \ldots(2 q+2 p-3)^{p}(2 q+2 p-1)^{p+1}(2 q+2 p+1)^{p+1}(2 q+2 p+3)^{p+1}(2 q+2 p+5)^{p} \ldots(2 q+4 p+1)^{2}(2 q+4 p+3)}, \\
& \text { which is what we wanted to prove. }
\end{aligned}
$$

(4) When the values of $\Delta$ and $D$ are introduced in (42) we get

$$
\begin{aligned}
& { }_{p}^{q}=\frac{\left\{1^{p-1} \cdot 2^{p-2} \ldots(p-2)^{2}(p-1)\right\}^{2} \cdot 2^{p(p-1)}}{(2 q-1)(2 q+1)^{2} \ldots(2 q+2 p-5)^{p-1}(2 q+2 p-3)^{p}(2 q+2 p-1)^{p-1} \ldots(2 q+4 p-7)^{2}(2 q+4 p-5)} \\
& +a \cdot 2^{3(p-1)} \times \\
& \frac{\left\{1^{p-1} \cdot 2^{p-2} \ldots(p-2)^{2}(p-1)\right\}^{2} \cdot 2^{(p-1)(p-3)} \cdot p}{(2 q-1)(2 q+1)^{2} \ldots(2 q+2 p-7)^{p-2}(2 q+2 p-5)^{p-1}(2 q+2 p-3)^{p-1}(2 q+2 p-1)^{p-1}(2 q+2 p+1)^{p-2} \cdots(2 q+4 p-7)^{2}(2 q+4 p-5)} \\
& \text { or } \quad{ }_{p}^{q} \delta=\frac{\left\{1^{p-1} \cdot 2^{p-2} \ldots(p-2)^{2}(p-1)\right\}^{2} \cdot 2^{p(p-1)}[1+a p(2 q+2 p-3)]}{(2 q-1)(2 q+1)^{2} \ldots(2 q+2 p-5)^{p-1}(2 q+2 p-3)^{p}(2 q+2 p-1)^{p-1} \ldots(2 q+4 p-7)^{2}(2 q+4 p-5)} \cdots \cdots \cdots \cdots \cdots(45) .
\end{aligned}
$$

The denominators of the formulae (38)-(41) for ${ }_{n} \sigma_{y}^{2}$ are now known since they only consist of the factors ${ }_{p}^{1} \delta$ and ${ }_{p}^{2} \delta$. To be able to write down the general expression for ${ }_{n} \sigma_{y}^{2}$ we should have to evaluate the minors of $\delta$, but their form is so complicated that a direct calculation of the determinants for the degrees of function in question appears to be simpler. With the material in hand we are however able to determine ${ }_{n} \sigma_{y}^{2}$ for $x=0$ and $x^{2}=1$.

(5) From (38) and (39) we see that

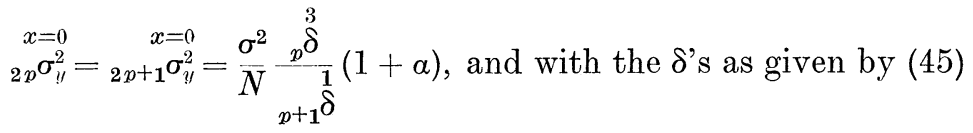

$$
\begin{aligned}
& \begin{array}{cc}
x=0 & x=0 \\
2 p & \sigma_{y}^{2}= \\
{ }_{2 p+1} \sigma_{y}^{2}
\end{array} \\
& \sigma^{2}(1+a)[1+a p(2 p+3)] 1 \cdot 3^{2} \cdot 5^{3} \cdot 7^{4} \cdot 9^{5} \ldots(2 p-1)^{p}(2 p+1)^{p+1}(2 p+3)^{p}(2 p+5)^{p-1} \ldots(4 p-1)^{2}(4 p+1) \\
& \overline{N\{1.2 .3 \ldots p\}^{2} \cdot 2^{2: 2} \cdot[1+a(p+1)(2 p+1)] 5 \cdot 7^{2} \cdot 9^{3} \ldots(2 p-1)^{p-2}(2 p+1)^{p-1}(2 p+3)^{p}(2 p+5)^{p-1} \ldots\left(4 p-3^{2}(4 p+1)\right.} \\
& =\frac{\sigma^{2}(1+\alpha) 3^{2} \cdot 5^{2} \ldots(2 p-1)^{2}(2 p+1)^{2} \cdot[1+\alpha p(2 p+3)]}{N\{1.2 .3 \ldots p\}^{2} \cdot 2^{2 p} \cdot[1+\alpha(p+1)(2 p+1)]} \\
& \text { or } \quad{ }_{2 p}^{x=0} \sigma_{y}^{2}={ }_{2 p+1}^{x=0} \sigma_{y}^{2}=\frac{\sigma^{2}}{N}\left\{\frac{3}{2} \cdot \frac{5}{4} \ldots \frac{2 p-1}{2 p-2} \cdot \frac{2 p+1}{2 p}\right\}^{2} \frac{(1+\alpha)[1+\alpha p(2 p+3)]}{[1+\alpha(p+1)(2 p+1)]} \text {. }
\end{aligned}
$$

(6) To find ${ }_{n}^{x^{2}=1} \sigma_{y}^{2}$ we have to evaluate the determinant of $(p+1)$ st order,

$$
\left|\begin{array}{ccccc}
0 & 1 & 1 & \cdots \cdots & 1 \\
1 & \frac{1}{2 q-1}+\alpha & \frac{1}{2 q+1}+\alpha & \cdots \cdots & \frac{1}{2 q+2 p-3}+\alpha \\
1 & \frac{1}{2 q+1}+\alpha & \frac{1}{2 q+3}+\alpha & \cdots \cdots & \frac{1}{2 q+2 p-1}+\alpha \\
\vdots & \vdots & \vdots & \vdots \\
1 & \frac{1}{2 q+2 p-3}+\alpha & \frac{1}{2 q+2 p-1}+\alpha \cdots \cdots & 1 \\
& \vdots & 2 q+4 p-5
\end{array}\right| .
$$


Treating it as $\underset{p}{\stackrel{q}{\delta}}$ was treated under (2) of this section, except that now two rows or columns are left unaltered, it takes the form

$$
\begin{array}{|cccccc}
0 & 1 & 0 & 0 & \cdots \cdots & 0 \\
1 & \frac{1}{2 q-1}+a & \frac{2}{(2 q-1)(2 q+1)} & \frac{2}{(2 q+1)(2 q+3)} & \cdots \cdots & \frac{2}{(2 q+2 p-5)(2 q+2 p-3)} \\
0 & \frac{2}{(2 q-1)(2 q+1)} & \frac{2.4}{(2 q-1)(2 q+1)(2 q+3)} & \frac{2.4}{(2 q+1)(2 q+3)(2 q+5)} & \cdots \cdots & \frac{2.4}{(2 q+2 p-5) \ldots(2 q+2 p-1)} \\
0 & \frac{2}{(2 q+1)(2 q+3)} & \frac{2.4}{(2 q+1)(2 q+3)(2 q+5)} & \frac{2.4}{(2 q+3)(2 q+5)(2 q+7)} & \cdots \cdots \frac{2.4}{(2 q+2 p-3) \ldots(2 q+2 p+1)} \\
\vdots & \vdots & \vdots & \vdots & \vdots \\
0 & \frac{2}{(2 q+2 p-5)(2 q+2 p-3)} & \frac{2.4}{(2 q+2 p-5) \ldots(2 q+2 p-1)} & \frac{2.4}{(2 q+2 p-3) \ldots(2 q+2 p+1)} \cdots \cdots \frac{2.4}{(2 q+4 p-9) \ldots(2 q+4 p-5)}
\end{array} \mid
$$

Hence we find from (38),

$$
{ }_{2 p}^{x^{2}=1} \sigma_{y}^{2}=\frac{\sigma^{2}}{N}(1+a)\left\{\frac{2^{3 p} \stackrel{1}{D} \frac{2^{3(p-1)}{ }_{p-1} \stackrel{2}{D}}{{ }_{p+1} \delta}}{{ }^{2}}\right\}
$$

Now from (43) and (45) we get

and therefore

$$
\frac{2^{3 p}{ }_{p} \stackrel{q}{D}}{{ }_{p+1}^{q}}=\frac{(p+1)(2 q+2 p-1)}{[1+a(p+1)(2 q+2 p-1)]}
$$

$$
\begin{aligned}
x^{2}=1 & =\sigma^{2} \\
{ }_{2} \sigma_{y}^{2} & \left.=\frac{(p+1)(2 p+1)}{N}+\frac{p(2 p+1)}{1+\alpha p(2 p+1)}\right\} \\
\text { or } \quad \begin{array}{c}
x^{2}=1 \\
{ }_{2 p} \sigma_{y}^{2}
\end{array} & =\frac{\sigma^{2}}{N}(1+\alpha)(2 p+1)\left\{\begin{array}{c}
p+1 \\
1+\alpha(p+1)(2 p+1)
\end{array}+\frac{p}{1+\alpha p(2 p+1)}\right\} \ldots .
\end{aligned}
$$

In the same way we get from (39),

$$
{ }_{2 p-1}^{x^{2}=1} \sigma_{\|}^{2}=\frac{\sigma^{2}}{N}(1+\alpha)\left\{\frac{\left\{2^{3(p-1)}{ }_{p-1} \stackrel{1}{D}\right.}{{ }_{p}^{1} \delta}+\frac{2^{3(p-1)}{ }_{p-1} \stackrel{2}{D}}{{ }_{p}^{2}}\right\},
$$

which by the relation between ${ }_{p} \stackrel{q}{D}$ and ${ }_{p+1}{ }^{q}$ just found is reduced to

$$
\underset{2 p-1}{x^{2}=1} \sigma_{y}^{2}=:=\frac{\sigma^{2}}{N}(1+\alpha)\left\{\frac{p(2 p-1)}{1+\alpha p(2 p-1)}+\frac{p(2 p+1)}{1+\alpha p(2 p+1)}\right\}
$$

Both (48) and (49) are covered by the formula

$$
{ }_{n}^{x^{2}=1} \sigma_{y}^{2}=\frac{\sigma^{2}}{N}(1+\alpha)(n+1)\left\{\frac{n}{2+a n(n+1)}+\frac{n+2}{2+\alpha(n+1)(n+2)}\right\} \ldots
$$

(7) The evaluation of ${ }_{n} \sigma_{\eta !}^{2}$ for special values of $n$ can be made easier by a transformation of the determinant 
KIRSTINe SMith

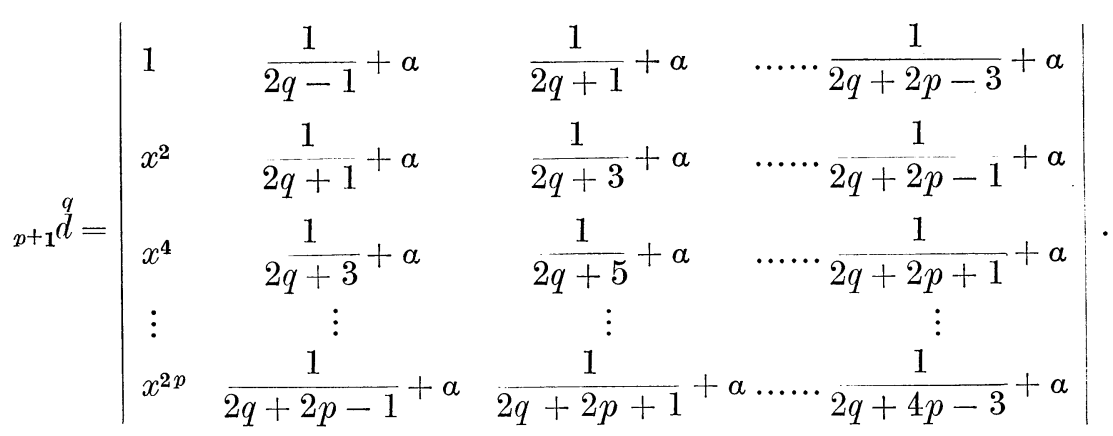

Leaving the first row unaltered and subtracting from each of the others the proceeding we get a determinant the first column of which is

$$
1, x^{2}-1, x^{2}\left(x^{2}-1\right) \ldots x^{2 p-2}\left(x^{2}-1\right)
$$

while the other columns are identical with those of the determinant $\delta$ previously treated in the same way. When next the two first rows are left as they are and from each of the others is subtracted the proceeding one the result is

${ }_{p+1}^{q} \stackrel{d}{d}=(-1)^{2 p-1} \times$

$$
\begin{array}{|ccccc}
1 & \frac{1}{2 q-1}+\alpha & \frac{1}{2 q+1}+\alpha & \cdots & \frac{1}{2 q+2 p-3}+a \\
1-x^{2} & \frac{2}{(2 q-1)(2 q+1)} & (2 q+1)(2 q+3) & \cdots & \frac{2}{(2 q+2 p-3)(2 q+2 p-1)} \\
\left(1-x^{2}\right)^{2} & \frac{2.4}{(2 q-1)(2 q+1)(2 q+3)} & \frac{2.4}{(2 q+1)(2 q+3)(2 q+5)} & \cdots & \frac{2.4}{(2 q+2 p-3) \ldots(2 q+2 p+1)} \\
\vdots & \vdots & \vdots & 2.4 \\
x^{2 p-4}\left(1-x^{2}\right)^{2} & \frac{2.4}{(2 q+2 p-5) \ldots(2 q+2 p-1)} & \frac{2.4}{(2 q+2 p-3) \ldots(2 q+2 p+1)} \cdots \frac{2.4}{(2 q+4 p-7) \ldots(2 q+4 p-3)}
\end{array} \mid .
$$

Leaving now three rows unaltered, next time four and so on, it is clear that we shall at last after $p$ of these sets of operations get

$$
\begin{aligned}
& \stackrel{q}{d}=(-1)^{\frac{p(p+1)}{2}} \times \\
& 1 \quad \frac{1}{2 q-1}+\alpha \\
& 1-x^{2} \quad \frac{2}{(2 q-1)(2 q+1)} \\
& \frac{1}{2 q+1}+\alpha \quad \ldots \quad \frac{1}{2 q+2 p-3}+\alpha \\
& \left(1-x^{2}\right)^{2} \frac{2.4}{(2 q-1)(2 q+1)(2 q+3)} \\
& \text { : } \\
& \left(1-x^{2}\right)^{p} \frac{2.4 \ldots 2 p}{(2 q-1) \ldots \ldots(2 q+2 p-1)} \\
& 2 \\
& \overline{(2 q+1)(2 q+3)} \quad \cdots \quad \overline{(2 q+2 p-3)(2 q+2 p-1)} \\
& \frac{2.4}{(2 q+1)(2 q+3)(2 q+5)} \cdots \frac{2.4}{(2 q+2 p-3) \ldots \ldots(2 q+2 p+1)} \text {. }
\end{aligned}
$$

By treating the columns in the same way, leaving first two then three and so on unaltered, we find after the first set of operations 


$$
\begin{aligned}
& { }_{p+1}^{q} \stackrel{q}{d}=(-1)^{\frac{p(p+1)}{2}+(p-1)} \times
\end{aligned}
$$

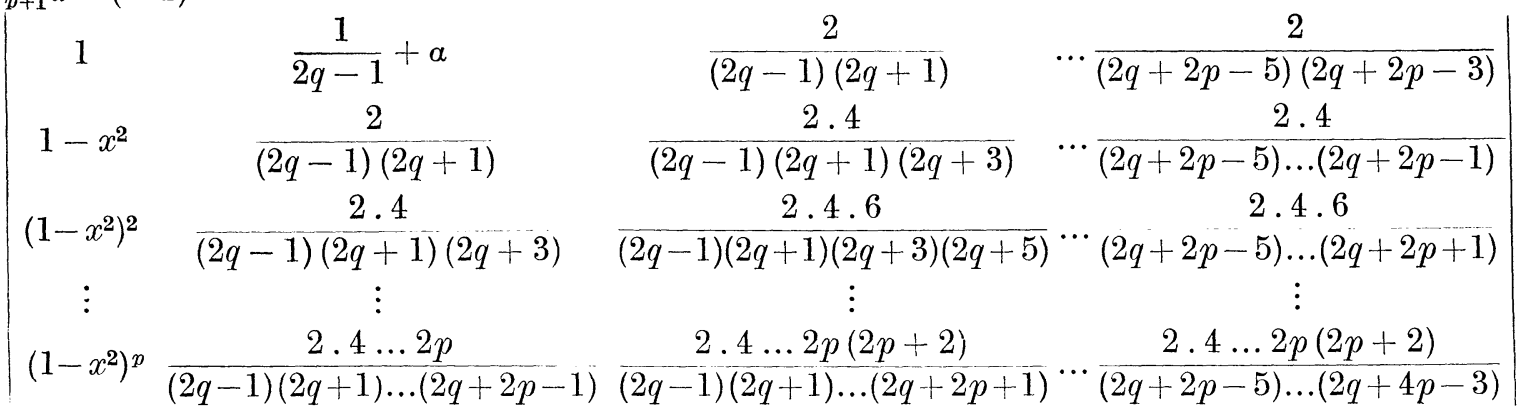

$$
\begin{aligned}
& \text { and after }(p-1) \text { sets of operations } \\
& { }_{p+1}^{\stackrel{q}{d}}=(-1)^{p^{2}} \times
\end{aligned}
$$

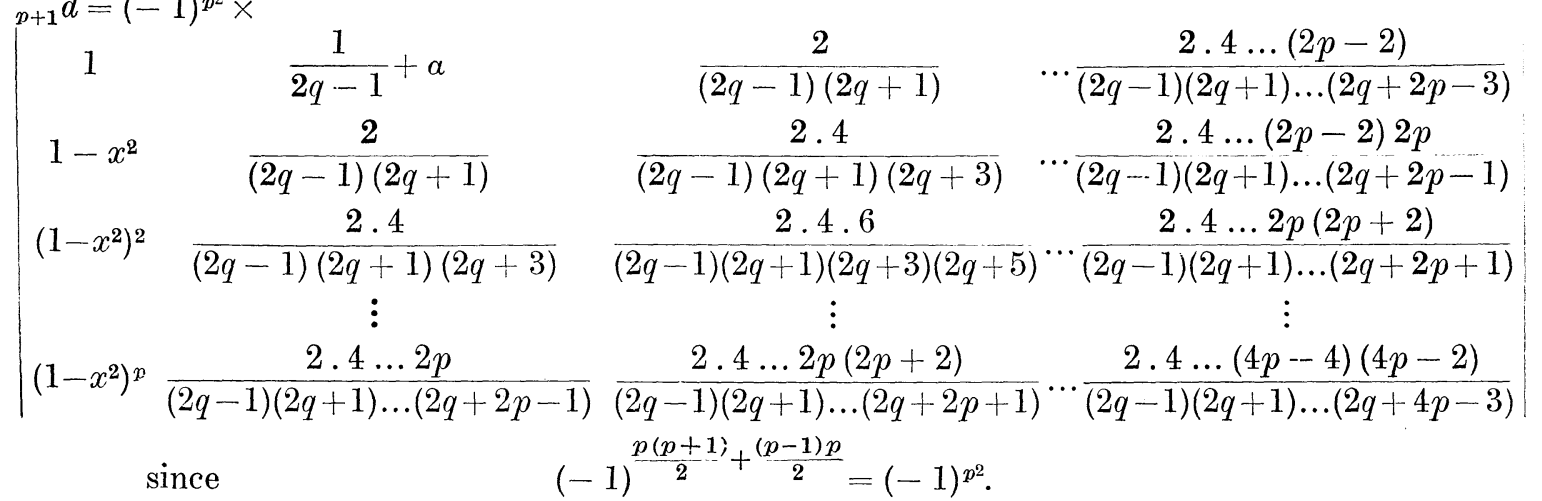

Here the first element of the last $p-1$ columns is seen to occur as factor for the whole column so that we can put outside the factor

$$
\begin{aligned}
& \frac{2^{p-1} \cdot 4^{p-2} \ldots(2 p-4)^{2}(2 p-2)}{(2 q-1)^{p-1}(2 q+1)^{p-1}(2 q+3)^{p-2}(2 q+5)^{p-3} \ldots(2 q+2 p-5)^{2}(2 q+2 p-3)} \\
& \quad=\frac{1^{p-1} \cdot 2^{p-2} \ldots(p-2)^{2}(p-1) 2^{\frac{p(p-1)}{2}}}{(2 q-1)^{p-1}(2 q+1)^{p-1}(2 q+3)^{p-2}(2 q+5)^{p-3} \ldots(2 q+2 p-5)^{2}(2 q+2 p-3)}
\end{aligned}
$$

the resulting expression being

$$
\begin{aligned}
& { }_{p+1}^{q} \stackrel{q}{d=}=\frac{(-1)^{p^{2}} \cdot 1^{p-1} \cdot 2^{p-2} \ldots(p-2)^{2}(p-1) 2^{\frac{p(p-1)}{2}}}{(2 q-1)^{p-1}(2 q+1)^{p-1}(2 q+3)^{p-2} \ldots(2 q+2 p-5)^{2}(2 q+2 p-3)} \times \\
& \begin{array}{|ccccc|}
1 & \frac{1}{2 q-1}+\alpha & 1 & \ldots & 1 \\
1-x^{2} & \frac{2}{(2 q-1)(2 q+1)} & \frac{4}{2 q+3} & \ldots & \frac{2 p}{2 q+2 p-1} \\
& 2.4 & \frac{4.6}{(2 q+3)(2 q+5)} & \cdots & \frac{2 p(2 p+2)}{(2 q+2 p-1)(2 q+2 p+1)} \\
\left(1-x^{2}\right)^{2} & \frac{2 q}{(2 q-1)(2 q+1)(2 q+3)} & \vdots & & \vdots \\
\vdots & \vdots & \frac{4.6 \ldots(2 p+2)}{(2 q+3) \ldots(2 q+2 p+1)} & \cdots & \frac{2 p \ldots(4 p-2)}{(2 q+2 p-1) \ldots(2 q+4 p-3)} \\
\left(1-x^{2}\right)^{p} & \frac{2.4 \ldots 2 p}{(2 q-1)(2 q+1) \ldots(2 q+2 p-1)} & \frac{1}{(2 q+3 q}
\end{array}
\end{aligned}
$$


In our formulae the two cases $q=1$ or $q=2$ only occur for which according to this we find

$$
\begin{aligned}
& { }_{p+1} \stackrel{1}{d}=\frac{(-1)^{p^{2}} \cdot 1^{p-1} \cdot 2^{p-2} \ldots(p-2)^{2}(p-1) 2^{p(p-1)}}{3^{p-1} \cdot 5^{p-2} \cdot 7^{p-3} \ldots(2 p-3)^{2}(2 p-1)} \times \\
& \begin{array}{|cccccc|}
1 & 1+a & 1 & 1 & \ldots & 1 \\
1-x^{2} & \frac{2}{1.3} & \frac{4}{5} & \frac{6}{7} & \ldots & \frac{2 p}{2 p+1}
\end{array} \\
& \begin{array}{lllllc}
\left(1-x^{2}\right)^{2} & \frac{2.4}{1.3 .5} & 4.6 & \frac{6.8}{7.7} & & 2 p(2 p+2) \\
7.9 & \cdots & (2 p+1)(2 p+3)
\end{array} \\
& \begin{array}{lllll}
\left(1-x^{2}\right)^{3} & \frac{2.4 .6}{1.3 .5 .7} & \frac{4.6 .8}{5.7 .9} & \frac{6.8 .10}{7.9 .11} & \ldots \frac{2 p(2 p+2)(2 p+4)}{(2 p+1)(2 p+3)(2 p+5)}
\end{array} \\
& \begin{array}{ccccc}
\vdots & \vdots & \vdots & \vdots & \vdots \\
\left(1-x^{2}\right)^{p} & \frac{2.4 \ldots 2 p}{1.3 \ldots(2 p+1)} & \frac{4.6 \ldots(2 p+2)}{5.7 \ldots(2 p+3)} & \frac{6.8 \ldots(2 p+4)}{7.9 \ldots(2 p+5)} & \ldots \frac{2 p(2 p+2) \ldots(4 p-2)}{(2 p+1)(2 p+3) \ldots(4 p-1)}
\end{array}
\end{aligned}
$$

and

$$
\begin{aligned}
& \stackrel{2}{d}=\frac{(-1)^{p^{2}} \cdot 1^{p-1} \cdot 2^{p-2} \ldots(p-2)^{2}(p-1) 2^{\frac{p(p-1)}{2}}}{3^{p-1} \cdot 5^{p-1} \cdot 7^{p-2} \ldots(2 p-1)^{2}(2 p+1)} \times \\
& \left|\begin{array}{cccccc}
1 & \frac{1}{3}+\alpha & 1 & 1 & \ldots & 1 \\
1-x^{2} & \frac{2}{3.5} & \frac{4}{7} & \frac{6}{9} & \ldots & \frac{2 p}{2 p+3} \\
\left(1-x^{2}\right)^{2} & \frac{2.4}{3.5 .7} & 4.6 & \frac{6.8}{9.11} & \ldots & \frac{2 p(2 p+2)}{2 p+3)(2 p+5)} \\
\left(1-x^{2}\right)^{3} & 2.4 .6 & 4.6 .8 & 6.8 .10 & \ldots \frac{2 p(2 p+2)(2 p+4)}{(2 p+3)(2 p+5)(2 p+7)} \\
\vdots & 3.5 .7 .9 & 7.9 .11 & 9.11 .13 & \cdots & \vdots \\
\left(1-x^{2}\right)^{p} & \frac{2.4 .6 \ldots 2 p}{3.5 .7 \ldots(2 p+3)} & \frac{4.6 \ldots(2 p+2)}{7.9 \ldots(2 p+5)} & \frac{6.8 \ldots(2 p+4)}{9.11 \ldots(2 p+7)} & \cdots \frac{2 p(2 p+2) \ldots(4 p-2)}{(2 p+3)(2 p+5) \ldots(4 p+1)}
\end{array}\right|
\end{aligned}
$$

VI. Uniform continuous distribution of observations with additional clusters at the ends of the range; constant standard deviation of observations. Special formulae.

(1) Our first task shall be to work out the formulae for ${ }_{n} \sigma_{y}^{2}-{ }_{n-1} \sigma_{y}^{2}$ for values of $n$ up to 6 , the next to find what values should be given to $\alpha$ in order to make ${ }_{n} \sigma_{y}^{2}$ as flat a curve as possible within the range of observations.

With the notations just introduced (40) and (41) take the form

$$
S_{2 p}={ }_{2 p} \sigma_{y}^{2}-{ }_{2 p-1} \sigma_{y}^{2}=\frac{\sigma^{2}}{N}(1+a) \frac{{ }_{p+1}^{1} d^{2}}{{ }_{p}^{1} \delta \cdot{ }_{p+1}{ }^{1}}
$$


and

$$
S_{2 p+1}={ }_{2 p+1} \sigma_{y}^{2}-{ }_{2 p} \sigma_{y}^{2}=\frac{\sigma^{2}}{N}(1+\alpha) x^{2} \frac{\stackrel{p+1}{2} d^{2}}{{ }_{p}^{2}{ }_{p+1}^{2} \delta} .
$$

From these formulae we find, after applying (45), (51) and (52), $S_{1}=\frac{\sigma^{2}}{N} \frac{3(1+\alpha) x^{2}}{1+3 \alpha}$

$$
\begin{array}{r}
S_{2}=\frac{\sigma^{2}}{N}(1+\alpha) \frac{1}{1+1.1 \alpha} \cdot \frac{1.3^{2} .5}{2^{2}(1+2.3 \alpha)}\left|\begin{array}{cc}
1 & 1+\alpha \\
1-x^{2} & \frac{2}{3}
\end{array}\right|^{2} \\
=\frac{\sigma^{2}}{N} \cdot \frac{5}{2^{2}} \frac{\left[2+3(1+\alpha)\left(x^{2}-1\right)\right]^{2}}{1+6 \alpha} .
\end{array}
$$

$$
\begin{aligned}
& S_{3}=\frac{\sigma^{2}}{N}(1+\alpha) x^{2} \frac{3}{1+1.3 \alpha} \cdot \frac{3.5^{2} .7}{2^{2}(1+2.5 \alpha)}\left|\begin{array}{cc}
1 & \frac{1}{3}+\alpha \\
1-x^{2} & \frac{2}{3.5}
\end{array}\right|^{2} \\
& =\frac{\sigma^{2}}{N} \cdot \frac{7}{2^{2}} \frac{(1+\alpha) x^{2}\left[2+5(1+3 \alpha)\left(x^{2}-1\right)\right]^{2}}{(1+3 \alpha)(1+10 \alpha)}
\end{aligned}
$$

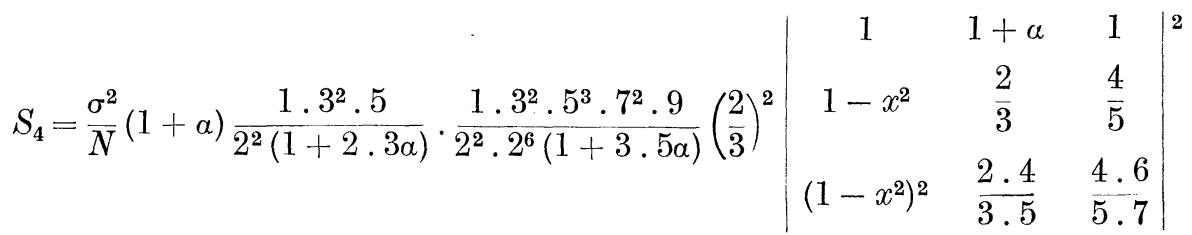$$
=\frac{\sigma^{2}}{N} \cdot \frac{9}{2^{6}} \frac{(1+\alpha)\left[8+20(2+9 \alpha)\left(x^{2}-1\right)+35(1+6 \alpha)\left(x^{2}-1\right)^{2}\right]^{2}}{(1+6 \alpha)(1+15 \alpha)}
$$

$$
\begin{aligned}
& S_{5}=\frac{\sigma^{2}}{N}(1+\alpha) \frac{3.5^{2} .7}{2^{2}(1+2.5 \alpha)} \cdot \frac{3.5^{2} \cdot 7^{3} \cdot 9^{2} .11}{2^{2} \cdot 2^{6}(1+3.7 \alpha)}\left(\frac{2}{3.5}\right)^{2} x^{2}\left|\begin{array}{ccc}
1 & \frac{1}{3}+\alpha & 1 \\
1-x^{2} & \frac{2}{3.5} & \frac{4}{7} \\
\left(1-x^{2}\right)^{2} & 2.4 & \frac{4.6}{7.5}
\end{array}\right|^{2} \\
& =\frac{\sigma^{2}}{\bar{N}} \cdot \frac{11}{2^{6}} \frac{(1+\alpha) x^{2}\left[8+28(2+15 \alpha)\left(x^{2}-1\right)+63(1+10 \alpha)\left(x^{2}-1\right)^{2}\right]^{2}}{(1+10 \alpha)(1+21 \alpha)} \ldots . .(57), \\
& S_{6}={ }_{N}^{\sigma^{2}}(1+\alpha) \frac{1.3^{2} .5^{3} .7^{2} .9}{2^{2} .2^{6}(1+3.5 \alpha)} \cdot \frac{1.3^{2} .5^{3} .7^{4} .9^{3} .11^{2} .13}{\left(2^{2} .3\right)^{2} .2^{12}(1+4.7 \alpha)}\left(\frac{2.2^{3}}{3^{2} .5}\right)^{2}\left|\begin{array}{cccc}
1 & 1+\alpha & 1 & 1 \\
1-x^{2} & \frac{2}{3} & \frac{4}{5} & \frac{6}{7} \\
\left(1-x^{2}\right)^{2} & \frac{2.4}{3.5} & \frac{4.6}{5.7} & \frac{6.8}{7.9} \\
\left(1-x^{2}\right)^{3} & \frac{2.4 .6}{3.5 .7} & \frac{4.6 .8}{5.7 .9} & \frac{6.8 .10}{7.9 .11}
\end{array}\right|^{2} \\
& =\frac{\sigma^{2}}{N} \cdot \frac{13}{2^{8}} \frac{(1+\alpha)\left[16+168(1+10 \alpha)\left(x^{2}-1\right)+126(3+40 \alpha)\left(x^{2}-1\right)^{2}+231(1+15 \alpha)\left(x^{2}-1\right)^{3}\right]^{2}}{(1+15 \alpha)(1+28 \alpha)}
\end{aligned}
$$


(2) We shall now look at ${ }_{n} \sigma_{y}^{2}$ for special values of $n$ and as a first attempt at

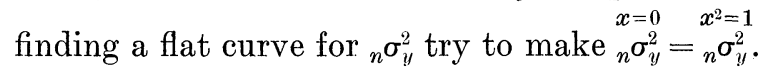

For a linear function we find, since

$$
\begin{aligned}
& { }_{1} \sigma_{y}^{2}={ }_{0} \sigma_{y}^{2}+S_{1}, \\
& { }_{1} \sigma_{y}^{2}=\frac{\sigma^{2}}{N}\left(1+\frac{3(1+\alpha)}{1+3 \alpha} x^{2}\right)
\end{aligned}
$$

As $\alpha$ is positive it is obvious that we cannot make $\begin{gathered}x=0 \\ { }_{1} \sigma_{y}^{2}\end{gathered}={ }_{1} \sigma_{1}^{2}=1$ which indeed we knew beforehand. This follows because we have proved that ${ }_{n} \sigma_{y}^{2}$ is of $2 n$th degree and never lower.

For $x=0$ we find

$$
{ }_{1}^{x=0} \sigma_{y}^{2}=\frac{\sigma^{2}}{N},
$$

which holds for any symmetrical distribution of observations with constant standard deviation. $a$ is the ratio between the number of observations at the ends of the range and the number uniformly distributed through the range, it may therefore vary from 0 to $\infty$. As $\frac{3(1+\alpha)}{1+3 \alpha}$ decreases when $\alpha$ increases we get the flattest possible curve when $\alpha=\infty$, that is when the distribution of observations consists of two groups at the ends of the range. Then the curve is, as already shown in Section II,

$$
{ }_{1} \sigma_{y}^{2}=\frac{\sigma^{2}}{N}\left(1+x^{2}\right) .
$$

To get a check on the degree of the function and at the same time a flatter curve of $\sigma_{y}^{2}$ than that obtained from a uniform distribution we may choose something between the two extreme cases and take for example $\frac{1}{4} N$ observations at each end of the range and $\frac{1}{2} N$ uniformly distributed through the range.

Then $\alpha=1$ and, according to (59),

$$
\begin{aligned}
\sigma_{y}^{2} & =\frac{\sigma^{2}}{N}\left(1+\frac{3}{2} x^{2}\right), \\
x^{2}=1 & =\frac{\sigma}{\sqrt{ } N} \cdot 1 \cdot 581 . \\
\sigma_{\vartheta} &
\end{aligned}
$$

with the maximum

(3) For a function of the second degree we find, from (46),

and from (50),

$$
\begin{gathered}
{ }_{2}^{x=0} \sigma_{y}^{2}=\frac{\sigma^{2}}{N} \cdot \frac{9}{4} \frac{(1+\alpha)(1+5 \alpha)}{1+6 \alpha}, \\
{ }_{2}^{x^{2}=1} \sigma_{y}^{2}=\frac{\sigma^{2}}{N} 3(1+\alpha)\left\{\frac{1}{1+3 \alpha}+\frac{2}{1+6 \alpha}\right\} .
\end{gathered}
$$

We want to make these equal and this requires

or

$$
3(1+5 \alpha)(1+3 \alpha)=4\{1+6 \alpha+2(1+3 \alpha)\}
$$

$$
15 a^{2}-8 a-3=0 \text {. }
$$

This has only one positive root $\alpha=\cdot 7873500$. 
For this value $\frac{\alpha}{2(1+\alpha)}$, which is the ratio between the number of observations at one end of the range and the total number of observations, is $\cdot 2202562$.

$\mathrm{As}_{2} \sigma_{y}^{2}={ }_{1} \sigma_{y}^{2}+S_{2}$ we find, from (59) and (54),

$$
{ }_{2} \sigma_{y}^{2}=\frac{\sigma^{2}}{N}\left(1+\frac{3(1+\alpha)}{1+3 \alpha} x^{2}+\frac{5}{4} \frac{\left[2+3(1+\alpha)\left(x^{2}-1\right)\right]^{2}}{1+6 \alpha}\right)
$$

for $\alpha=\cdot 7873500$ the curve is

which has minima at $\quad x= \pm \frac{1}{\sqrt{ } 2}$.

$$
{ }_{2} \sigma_{y}^{2}=\frac{\sigma^{2}}{N}\left\{3 \cdot 46837-6 \cdot 27862 x^{2}+6 \cdot 27862 x^{4}\right\},
$$

The extreme values in the range of observations are therefore

and

$$
\begin{aligned}
& \sigma_{y}=\frac{\sigma}{\sqrt{ } N} \cdot 1 \cdot 8624 \text { for } x=\left\{\begin{array}{l}
0 \\
\pm 1
\end{array}\right. \\
& \sigma_{y}=\frac{\sigma}{\sqrt{ } N} \cdot 1 \cdot 3779 \text { for } x= \pm \cdot 70711 .
\end{aligned}
$$

(4) For a function of the third degree we have, from (46),

$$
\begin{array}{r}
x=0 \\
{ }_{3}^{2} \sigma_{y}^{2}=\frac{\sigma^{2}}{N} \cdot \frac{9}{4} \frac{(1+\alpha)(1+5 \alpha)}{1+6 a}, \\
\text { and from (50), } \quad x_{3}^{2=1}{ }_{3}^{2} \sigma_{y y}^{2}=\frac{\sigma^{2}}{N} 2(1+\alpha)\left\{\frac{3}{1+6 a}+\frac{5}{1+10 \alpha}\right\}
\end{array}
$$

Hence the condition that they are equal is

or

$$
\begin{gathered}
9(1+5 \alpha)(1+10 \alpha)=32(2+15 \alpha) \\
90 \alpha^{2}-69 \alpha-11=0,
\end{gathered}
$$

with one positive root $\alpha=\cdot 9021461$.

From $(60)$ and $(55)$ we find

which for $\alpha=.9021461$ becomes

$$
\begin{aligned}
{ }_{3} \sigma_{y}^{2}=\frac{\sigma^{2}}{N}\left(1+\frac{3(1+\alpha)}{1+3 \alpha} x^{2}\right. & +\frac{5}{4} \frac{\left[2+3(1+\alpha)\left(x^{2}-1\right)\right]^{2}}{1+6 \alpha} \\
& \left.+\frac{7}{4} \frac{(1+\alpha) x^{2}\left[2+5(1+3 a)\left(x^{2}-1\right)\right]^{2}}{(1+3 \alpha)(1+10 \alpha)}\right)
\end{aligned}
$$

$$
{ }_{3} \sigma_{y}^{2}=\frac{\sigma^{2}}{N^{2}}\left\{3 \cdot 67775+17 \cdot 78799 x^{2}-48 \cdot 56651 x^{4}+30 \cdot 77852 x^{6}\right\} .
$$

Besides the minimum for $x=0$ this curve has other minima for $x^{2}=\cdot 815820$ and maxima for $x^{2}=\cdot 2361366$.

The maxima and minima are as follows:

$$
\begin{aligned}
& \text { For } x=\left\{\begin{aligned}
& \pm 1 \sigma_{y}=\frac{\sigma}{\sqrt{ } N} \cdot 1 \cdot 9177, \\
& 0
\end{aligned}\right. \\
& , \quad x= \pm \cdot 48594 \quad \sigma_{y}=\frac{\sigma}{\sqrt{ } N} \cdot 2 \cdot 3612, \\
& , \quad x= \pm \cdot 90323 \quad \sigma_{y}=\frac{\sigma}{\sqrt{ } N} \cdot 1 \cdot 6055 .
\end{aligned}
$$




\section{Kinstine SMith}

By choosing $\alpha=\cdot 9021461$, that is by taking $\cdot 237139 \times N$ observations at each end of the range, we seem therefore to have overshot our aim since the result is that we have got inside the range a maximum for $\sigma_{y}$ greater than the value obtained for $x= \pm 1$.

(5) Our next attempt shall be to make

$$
\begin{gathered}
x^{2}=1 \\
{ }_{3} \sigma_{y}^{2}=2{ }_{3} \sigma_{y}^{2}
\end{gathered} \text {. }
$$

It requires $\quad 9(1+5 \alpha)(1+10 \alpha)=16(2+15 \alpha)$

or

$$
450 \alpha^{2}-105 \alpha-23=0 .
$$

The only positive root is $\alpha=\cdot 3710723$ which gives the curve

$$
{ }_{3} \sigma_{y}^{2}=\frac{\sigma^{2}}{N}\left\{2 \cdot 730117+12 \cdot 89741 x^{2}-37 \cdot 07612 x^{4}+26 \cdot 90882 x^{6}\right\} .
$$

The maxima and minima are:

$$
\begin{aligned}
& \text { For } x=\quad .0000 \quad \sigma_{y}=\frac{\sigma}{\sqrt{ } N} \cdot 1 \cdot 652, \\
& , \quad x= \pm \cdot 4828 \quad \sigma_{y}=\frac{\sigma}{\sqrt{ } N} \cdot 2 \cdot 016, \\
& \text {, } x= \pm \cdot 8279 \quad \sigma_{y}=\frac{\sigma}{\sqrt{ } N} \cdot 1 \cdot 678, \\
& , \quad x= \pm 1 \cdot 0000 \quad \sigma_{y}=\frac{\sigma}{\sqrt{ } N} \cdot 2 \cdot 337 .
\end{aligned}
$$

This distribution of observations makes $\sigma_{y}$ for $x= \pm 1$ greater than the maximum at $x= \pm \cdot 4828$. By interpolation between these two cases we shall now try to find an $\alpha$, lying between those of our two trials, for which $\sigma_{y}$ for $x= \pm 1$ equals the maximum value of $\sigma_{y}$ which still may be expected at about $x=\cdot 48$.

(6) In our first attempt we found $\begin{gathered}x=1 \\ \sigma_{y}\end{gathered}=\frac{\sigma}{\sqrt{ } N} \cdot 1 \cdot 918$ and its difference from the maximum $\frac{\sigma}{\sqrt{ } N} \cdot 444$, in the second attempt $\sigma_{y}=\frac{\sigma}{\sqrt{ } N} \cdot 2 \cdot 337$ and its difference from the maximum $-\frac{\sigma}{\sqrt{ } N} \cdot 321$.

If the relation were linear this difference would be zero for

$$
\begin{gathered}
x=1 \\
\sigma_{y}
\end{gathered}=\frac{\sigma}{\sqrt{ } N} \cdot 2 \cdot 161 .
$$

The $a$ for which $\sigma_{y}$ takes this value is found by (61) which leads to

or

$$
8(1+\alpha)(2+15 \alpha)=2 \cdot 161^{2}(1+6 \alpha)(1+10 \alpha)
$$

with the positive root $a=\cdot 519$.

For this value (62) becomes

$$
{ }_{3} \sigma_{y}^{2}=\frac{\sigma^{2}}{N}\left\{2 \cdot 9866+14 \cdot 2364 x^{2}-40 \cdot 0058 x^{4}+27 \cdot 4521 x^{6}\right\} .
$$


The maxima and minima are:

$$
\begin{aligned}
& \text { For } x=\quad .0000 \quad \sigma_{y}=\frac{\sigma}{\sqrt{ } N} \cdot 1 \cdot 728, \\
& , \quad x= \pm \cdot 4843 \quad \sigma_{y}=\frac{\sigma}{\sqrt{ } N} \cdot 2 \cdot 116, \\
& , \quad x= \pm .8585 \quad \sigma_{y}=\frac{\sigma}{\sqrt{N}} \cdot 1 \cdot 655, \\
& \text {, } x= \pm 1 \cdot 0000 \quad \sigma_{y}=\frac{\sigma}{\sqrt{ } N} \cdot 2 \cdot 161,
\end{aligned}
$$

and this distribution which has $\cdot 1708 \times N$ observations at each end of the range may be considered satisfactory.

(7) From (46) and (50) we find, for a function of the fourth degree,

and

$$
{ }_{4}^{x=0} \sigma_{y}^{2}=\frac{\sigma^{2}}{N} \cdot \frac{225}{64} \frac{(1+\alpha)(1+14 \alpha)}{1+15 \alpha}
$$

which are equal when

$$
{ }_{4}^{x^{2}=1} \sigma_{y}^{2}=\frac{\sigma^{2}}{N} 5(1+\alpha)\left\{\frac{2}{1+10 \alpha}+\frac{3}{1+15 \alpha}\right\},
$$

or

$$
9(1+14 \alpha)(1+10 \alpha)=64(1+12 \alpha)
$$

that is when

$$
1260 \alpha^{2}-552 \alpha-55=0,
$$

$$
\alpha=\cdot 5217564 \text {. }
$$

The formula for ${ }_{4} \sigma_{y}^{2}$, found from (62) and (56), is

$$
\begin{gathered}
{ }_{4} \sigma_{y y}^{2}=\frac{\sigma^{2}}{N}\left\{1+\frac{3(1+\alpha)}{1+3 \alpha} x^{2}+\frac{5}{4} \frac{\left[2+3(1+\alpha)\left(x^{2}-1\right)\right]^{2}}{1+6 \alpha}+\frac{7}{4} \frac{(1+\alpha) x^{2}\left[2+5(1+3 \alpha)\left(x^{2}-1\right)\right]^{2}}{(1+3 \alpha)(1+10 \alpha)}\right. \\
\left.+\frac{9}{64} \frac{(1+\alpha)\left[8+20(2+9 \alpha)\left(x^{2}-1\right)+35(1+6 a)\left(x^{2}-1\right)^{2}\right]^{2}}{(1+6 \alpha)(1+15 \alpha)}\right\} \ldots \ldots(63) .
\end{gathered}
$$

For $\alpha=\cdot 5217564$ it becomes

$$
{ }_{4} \sigma_{y}^{2}=\frac{\sigma^{2}}{N}\left\{5 \cdot 03367-19 \cdot 72772 x^{2}+133 \cdot 01711 x^{4}-235 \cdot 96817 x^{6}+122 \cdot 67868 x^{8}\right\} .
$$

The maxima and minima are as follows:

$$
\begin{aligned}
& \text { For } x=\left\{\begin{array}{r}
0 \\
\pm 1
\end{array} \quad \sigma_{y}=\frac{\sigma}{\sqrt{ } N} \cdot 2 \cdot 244,\right. \\
& , \quad x= \pm \cdot 3130 \quad \sigma_{y}=\frac{\sigma}{\sqrt{ } N} \cdot 2 \cdot 041, \\
& , \quad x= \pm \cdot 6844 \quad \sigma_{y}=\frac{\sigma}{\sqrt{ } N} \cdot 2 \cdot 575, \\
& , \quad x= \pm .9361 \quad \sigma_{y}=\frac{\sigma}{\sqrt{ } N} \cdot 1 \cdot 856 .
\end{aligned}
$$

We have again as for the function of the third degree brought $\sigma_{y}{ }^{2}=1$ down below one of the maxima of ${ }_{4} \sigma_{y}$, although since ${ }_{4} \sigma_{y}$ has a maximum at $x=0$ the demand $x=0 \quad x^{2}=1$ that $\sigma_{y}=\sigma_{y}$ is not so exacting as for ${ }_{3} \sigma_{y}$ which has a minimum at $x=0$. 
(8) We shall next make ${ }_{4} \sigma_{y}^{2}=1.2671861_{4}^{x=0} \sigma_{y}^{2 *}$.

The condition obtained from (46) and (50) is

$$
9 \times 1 \cdot 2671861(1+10 \alpha)(1+14 \alpha)=64(1+12 \alpha)
$$

or

$$
\alpha^{2}-\cdot 3095773 \alpha-\cdot 032940969=0,
$$

with the only positive root $\quad \alpha=\cdot 3933269$.

Introducing this value of $\alpha$ in (63) we get

$$
{ }_{4} \sigma_{y}^{2}=\frac{\sigma^{2}}{N}\left\{4 \cdot 61918-18 \cdot 02388 x^{2}+122 \cdot 71833 x^{4}-220 \cdot 34099 x^{6}+116 \cdot 8807 x^{8}\right\} .
$$

The maxima and minima for this curve are:

$$
\begin{aligned}
& \text { At } x=0 \quad \sigma_{y}=\frac{\sigma}{\sqrt{ } N} \cdot 2 \cdot 149 \text {, } \\
& , x= \pm \cdot 3116 \quad \sigma_{y}=\frac{\sigma}{\sqrt{ } N} \cdot 1 \cdot 958, \\
& \text {, } x= \pm \cdot 6839 \sigma_{y}=\frac{\sigma}{\sqrt{ } N} \cdot 2 \cdot 467, \\
& \text {, } x= \pm .9214 \quad \sigma_{y}=\frac{\sigma}{\sqrt{ } N} \cdot 1 \cdot 913, \\
& , \quad x= \pm 1 \cdot 0000 \quad \sigma_{y}=\frac{\sigma}{\sqrt{ } N} \cdot 2 \cdot 419 .
\end{aligned}
$$

We have thus for $\alpha=\cdot 3933269$, that is by taking $\cdot 141147 \times N$ observations at

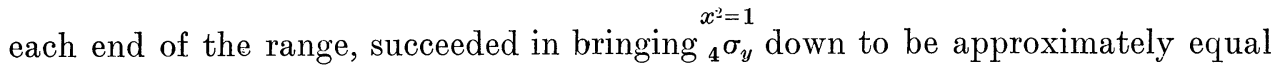
to the highest of the maxima of the curve, thus fulfilling our purpose.

(9) After our experiences in the cases of the functions of the third and fourth degree we cannot expect for a function of the fifth degree by making

$$
\begin{gathered}
x^{2}=1 \\
{ }_{5} \sigma_{y}^{2}={ }_{5}=0 \\
\sigma_{y}^{2}
\end{gathered}
$$

to find a curve which has not a greater maximum than that value. We shall therefore start with the attempt

$$
\begin{gathered}
x^{2}=1 \\
{ }_{5} \sigma_{y}^{2}
\end{gathered}=22_{5} \sigma_{y}^{2} .
$$

The condition found from (46) and (50) is

$$
25(1+14 \alpha)(1+21 \alpha)=64(2+35 \alpha)
$$

or

$$
7350 \alpha^{2}-1365 \alpha-103=0,
$$

with the only positive root $\quad \alpha=\cdot 2433100$.

* The ratio 1.2671861 results from consideration of a special ${ }_{2} \sigma_{y}^{2}$ curve. It was determined as that curve obtained from three groups of observations for which the standard deviation of $\sigma_{y}^{2}$ 's within the range of observations was a minimum. It is not mentioned elsewhere in this memoir as it does not seem to have the interest I at first assumed it to have. 


\section{Choice in the Distribution of Observations}

For ${ }_{5} \sigma_{y}^{2}$ we find, from (63) and (57),

$$
\begin{aligned}
{ }_{5} \sigma_{y}^{2}=\frac{\sigma^{2}}{N}\left\{1+\frac{3(1+\alpha)}{1+3 \alpha} x^{2}+\frac{5}{4} \frac{\left[2+3(1+\alpha)\left(x^{2}-1\right)\right]^{2}}{1+6 \alpha}+\frac{7}{4} \frac{(1+\alpha) x^{2}\left[2+5(1+3 \alpha)\left(x^{2}-1\right)\right]^{2}}{(1+3 \alpha)(1+10 \alpha)}\right. \\
+\frac{9}{64} \frac{(1+\alpha)\left[8+20(2+9 a)\left(x^{2}-1\right)+35(1+6 \alpha)\left(x^{2}-1\right)^{2}\right]^{2}}{(1+6 \alpha)(1+15 \alpha)} \\
\left.+\frac{11}{64} \frac{(1+\alpha) x^{2}\left[8+28(2+15 \alpha)\left(x^{2}-1\right)+63(1+10 \alpha)\left(x^{2}-1\right)^{2}\right]^{2}}{(1+10 \alpha)(1+21 \alpha)}\right\} \ldots(64)
\end{aligned}
$$

Introducing $\alpha=\cdot 2433100$ we get

$$
\begin{aligned}
{ }_{5} \sigma_{y}^{2}=\frac{\sigma^{2}}{N}\left\{4 \cdot 14228+28 \cdot 47030 x^{2}-258 \cdot 05238 x^{4}\right. & +853 \cdot 0448 x^{6}-1095 \cdot 921 x^{8} \\
& \left.+476 \cdot 5990 x^{10}\right\},
\end{aligned}
$$

from which we find the maxima and minima:

$$
\begin{aligned}
& \text { At } x=0 \quad \sigma_{y}=\frac{\sigma}{\sqrt{ } N} \cdot 2 \cdot 035 \\
& , x= \pm .2953 \quad \sigma_{y}=\frac{\sigma}{\sqrt{ } N} \cdot 2 \cdot 273 \\
& , x= \pm .5004 \quad \sigma_{y}=\frac{\sigma}{\sqrt{ } N} \cdot 2 \cdot 155 \\
& , x= \pm .7853 \quad \sigma_{y}=\frac{\sigma}{\sqrt{ } N} \cdot 2 \cdot 762 \\
& , x= \pm .9418 \quad \sigma_{y}=\frac{\sigma}{\sqrt{ } N} \cdot 2 \cdot 231 \\
& , x= \pm 1.0000 \quad \sigma_{y}=\frac{\sigma}{\sqrt{ } N} \cdot 2 \cdot 878
\end{aligned}
$$

$x^{2}=1$
$\sigma_{y}$ does not differ much from the greatest maximum and we may thus consider the distribution with $\cdot 097848 \times N$ observations at each end of the range for which $\alpha=\cdot 2433100$ as satisfying fairly well our aim.

(10) Considering our previous results we must assume that for a function of the sixth degree $\begin{gathered}x^{2}=1 \\ \sigma_{y}^{2}\end{gathered} / \begin{gathered}x=0 \\ \sigma_{y}^{2}\end{gathered}$ ought to be made somewhat smaller than 2 which was the value that gave a satisfying result for a function of the fifth degree.

Let us assume $\begin{gathered}x^{2}=1 \\ \sigma_{y}^{2}\end{gathered}=1 \cdot 75 \sigma_{y}^{x}$ or, substituting from (46) and (50),

$$
256(1+24 \alpha)=1.75 \times 25(1+21 \alpha)(1+27 \alpha)
$$

from which

$$
567 a^{2}-92 \cdot 43430 a-4 \cdot 851429=0
$$

and

$$
a=\cdot 2048019
$$

are found. 
Kirstine Smith

For ${ }_{6} \sigma_{y}^{2}$ we get, from (64) and (58),

$$
\begin{aligned}
{ }_{6} \sigma_{y}^{2}=\frac{\sigma^{2}}{N}\{1 & +\frac{3(1+\alpha)}{1+3 \alpha} x^{2}+\frac{5}{4} \frac{\left[2+3(1+\alpha)\left(x^{2}-1\right)\right]^{2}}{1+6 \alpha} \\
& +\frac{7}{4} \frac{(1+\alpha) x^{2}\left[2+5(1+3 \alpha)\left(x^{2}-1\right)\right]^{2}}{(1+3 \alpha)(1+10 \alpha)} \\
& +\frac{9}{64} \frac{1+\alpha}{(1+6 a)(1+15 \alpha)}\left[8+20(2+9 \alpha)\left(x^{2}-1\right)+35(1+6 \alpha)\left(x^{2}-1\right)^{2}\right]^{2} \\
& +\frac{11}{64} \frac{1+\alpha}{(1+10 \alpha)(1+21 \alpha)}\left[8+28(2+15 \alpha)\left(x^{2}-1\right)+63(1+10 \alpha)\left(x^{2}-1\right)^{2}\right]^{2} \\
& +\frac{13}{256} \frac{1+\alpha}{(1+15 \alpha)(1+28 \alpha)}\left[16+168(1+10 \alpha)\left(x^{2}-1\right)\right. \\
& \left.\left.\quad+126(3+40 \alpha)\left(x^{2}-1\right)^{2}+231(1+15 \alpha)\left(x^{2}-1\right)^{3}\right]^{2}\right\},
\end{aligned}
$$

which for $\alpha=\cdot 2048019$ becomes

${ }_{6} \sigma_{y}^{2}=\frac{\sigma^{2}}{N}\left\{5 \cdot 58984-33 \cdot 14234 x^{2}+504 \cdot 4523 x^{4}-2512 \cdot 673 x^{6}+5524 \cdot 186 x^{8}+\right.$

The maxima and minima are:

$$
\left.-5452 \cdot 650 x^{10}+1974 \cdot 020 x^{12}\right\} \text {. }
$$

$$
\begin{aligned}
& \text { At } x=0 \quad \sigma_{y}=\frac{\sigma}{\sqrt{ } N} \cdot 2 \cdot 364, \\
& , x= \pm \cdot 2216 \quad \sigma_{y}=\frac{\sigma}{\sqrt{ } N} \cdot 2 \cdot 216 \text {, } \\
& , x= \pm \cdot 4826 \quad \sigma_{y}=\frac{\sigma}{\sqrt{ } N} \cdot 2 \cdot 515, \\
& , x= \pm \cdot 6194 \quad \sigma_{y}=\frac{\sigma}{\sqrt{ } N} \cdot 2 \cdot 427 \text {, } \\
& \text {, } x= \pm \cdot 8445 \quad \sigma_{y}=\frac{\sigma}{\sqrt{ } N} \cdot 3 \cdot 149, \\
& , x= \pm .9615 \quad \sigma_{y}=\frac{\sigma}{\sqrt{ } N} \cdot 2 \cdot 485, \\
& , x= \pm 1 \cdot 0000 \quad \sigma_{y}=\frac{\sigma}{\sqrt{ } N} \cdot 3 \cdot 128 .
\end{aligned}
$$

It thus appears that this distribution which has $\cdot 08499 \times N$ observations at each end of the range fulfils our demand that $\sigma_{y}$ shall be approximately equal to the greatest of the maxima.

(11) We bring together our final results in the following table. It gives the distribution of observations, the maximum of $\sigma_{y}$ within the range, the value of $\sqrt{n+1}$ or the lowest maximum of $\frac{\sigma_{y} \sqrt{ } N}{\sigma}$ possible, which can only be obtained by distributing the observations of the function of the $n$th degree into $(n+1)$ groups, and the value of $n+1$ which is the maximum of $\frac{\sigma_{y} \sqrt{ } N}{\sigma}$ for a uniform distribution.

Biometrika XII 
TABLE II.

\begin{tabular}{|c|c|c|c|c|}
\hline $\begin{array}{c}\text { Degree of } \\
\text { function }\end{array}$ & $\begin{array}{c}\text { Ratio of number of } \\
\text { observations at each } \\
\text { end of the range to } \\
\text { the total number }\end{array}$ & $\begin{array}{c}\text { Maximum of } \\
\sigma_{y} \sqrt{ } N\end{array}$ & $\sqrt{n+1}$ & $n+1$ \\
\hline & -2500 & $1 \cdot 581$ & $1 \cdot 414$ & 2 \\
1 & $\cdot 2203$ & $1 \cdot 862$ & $1 \cdot 732$ & 3 \\
2 & $\cdot 1708$ & $2 \cdot 161$ & $2 \cdot 000$ & 4 \\
3 & $\cdot 1411$ & $2 \cdot 467$ & $2 \cdot 236$ & 5 \\
4 & $\cdot 0978$ & $2 \cdot 878$ & $2 \cdot 449$ & 6 \\
5 & $\cdot 0850$ & $3 \cdot 149$ & $2 \cdot 646$ & 7 \\
\hline
\end{tabular}

A comparison between our maximum and $\sqrt{n+1}$ shows the price we have to pay for information about the degree of the function. For lower degrees the maximum only differs quite insignificantly from $\sqrt{n+1}$, but with increasing degree the difference grows relatively greater for the sixth degree, being about one-fifth of $\sqrt{ } n+1$.

The curves of standard deviation for the three sets of distributions are given in Diagrams 3-8, while Diagram 9 represents the six curves just reached. It seems likely from the form of the $\sigma_{y}$ curves that two clusters of observations placed at the outermost of the maxima besides the two clusters at the ends of the range would produce a $\sigma_{y}$ curve with a lower maximum than the one we have succeeded in getting for the functions from the fourth to the sixth degree. But then again the position of these new clusters would depend on the degree of the function and thus make the proceedings more complicated; and what is more at the same time as the maximum of the curve approached $\sqrt{n+1}$ the distribution of observations would incur the disadvantages of the grouping in $(n+1)$ clusters. On the whole the distribution arrived at seems to be satisfactory and certainly marks a great progress from the uniform distribution.

\section{Observations with varying standard deviation.}

(1) In Section I we have already given the formula for the standard deviation $\sigma_{y}$ of an adjusted $y$ when the standard deviation $s_{y}$ of an observation is $\sigma \sqrt{f(x)}$.

It is

$$
\left|\begin{array}{cccccc}
\sigma_{y}^{2} \cdot \frac{N}{\sigma^{2}} & 1 & x & x^{2} & \ldots \ldots & x^{n} \\
1 & m_{0} & m_{1} & m_{2} & \ldots \ldots & m_{n} \\
x & m_{1} & m_{2} & m_{3} & \ldots \ldots & m_{n+1} \\
x^{2} & m_{2} & m_{3} & m_{4} & \ldots \ldots & m_{n+2} \\
\vdots & \vdots & \vdots & \vdots & & \vdots \\
x^{n} & m_{n} & m_{n+1} & m_{n+2} & \ldots \ldots & m_{2 n}
\end{array}\right|=0
$$




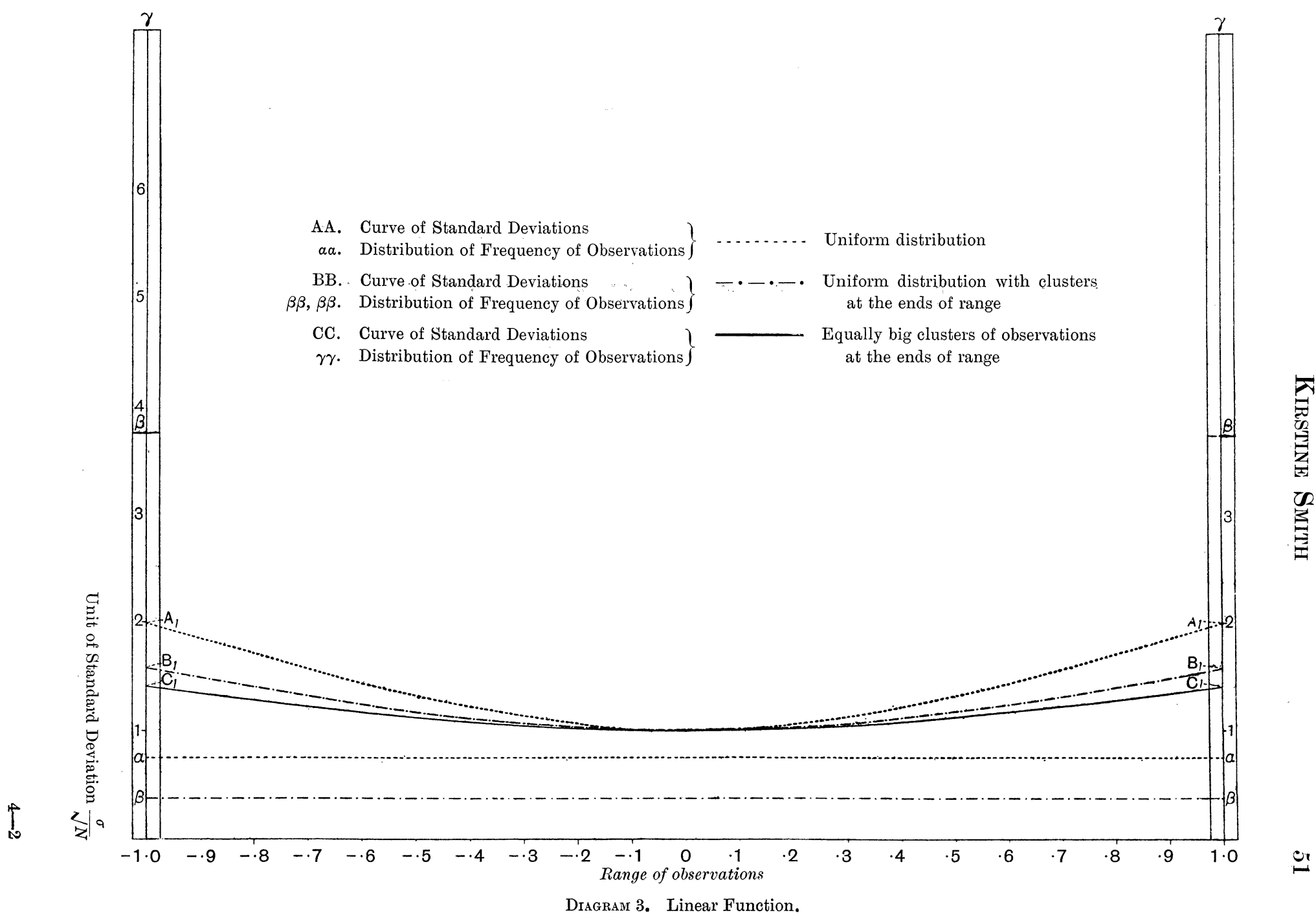




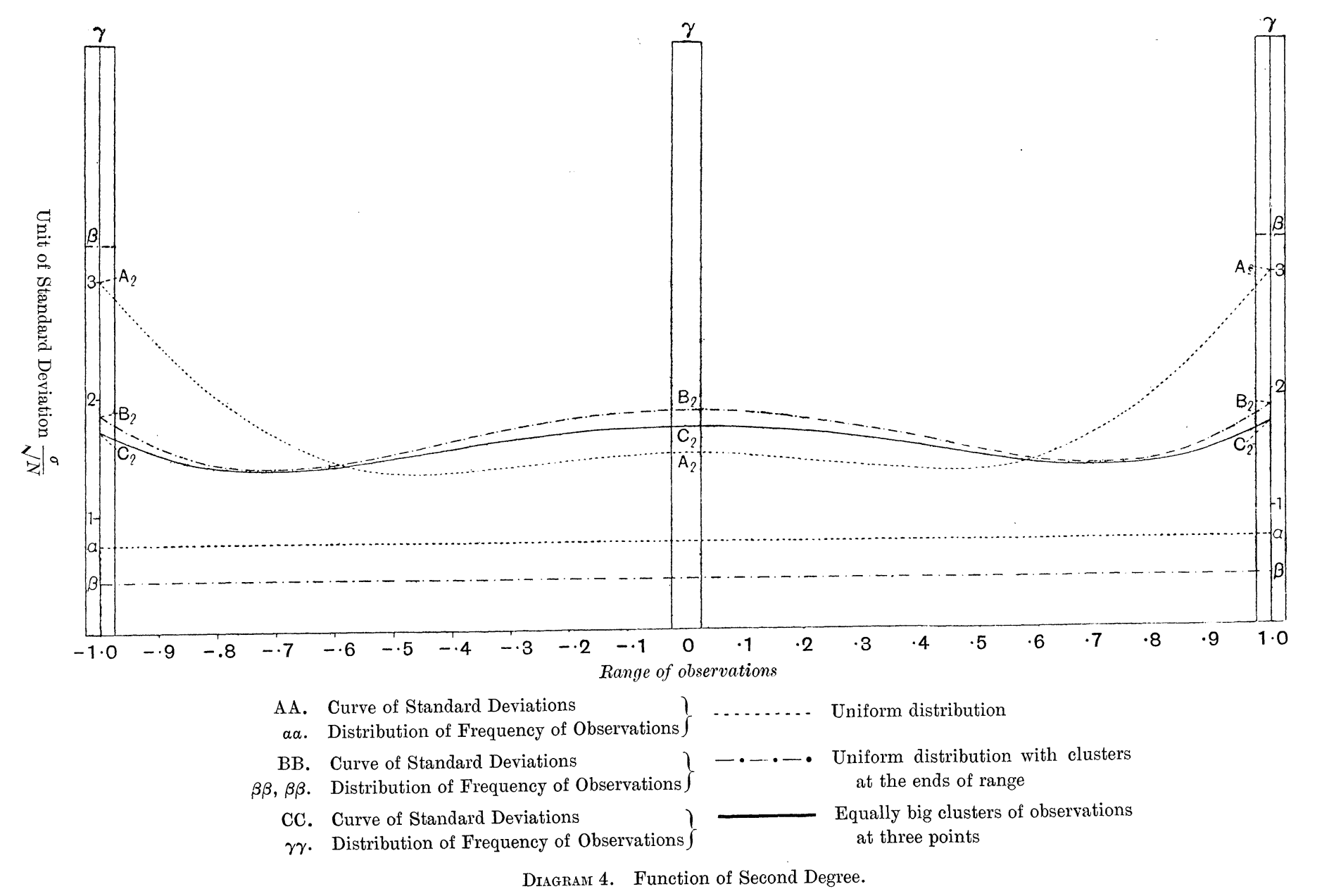

Diagram 4. Function of Second Degree. 


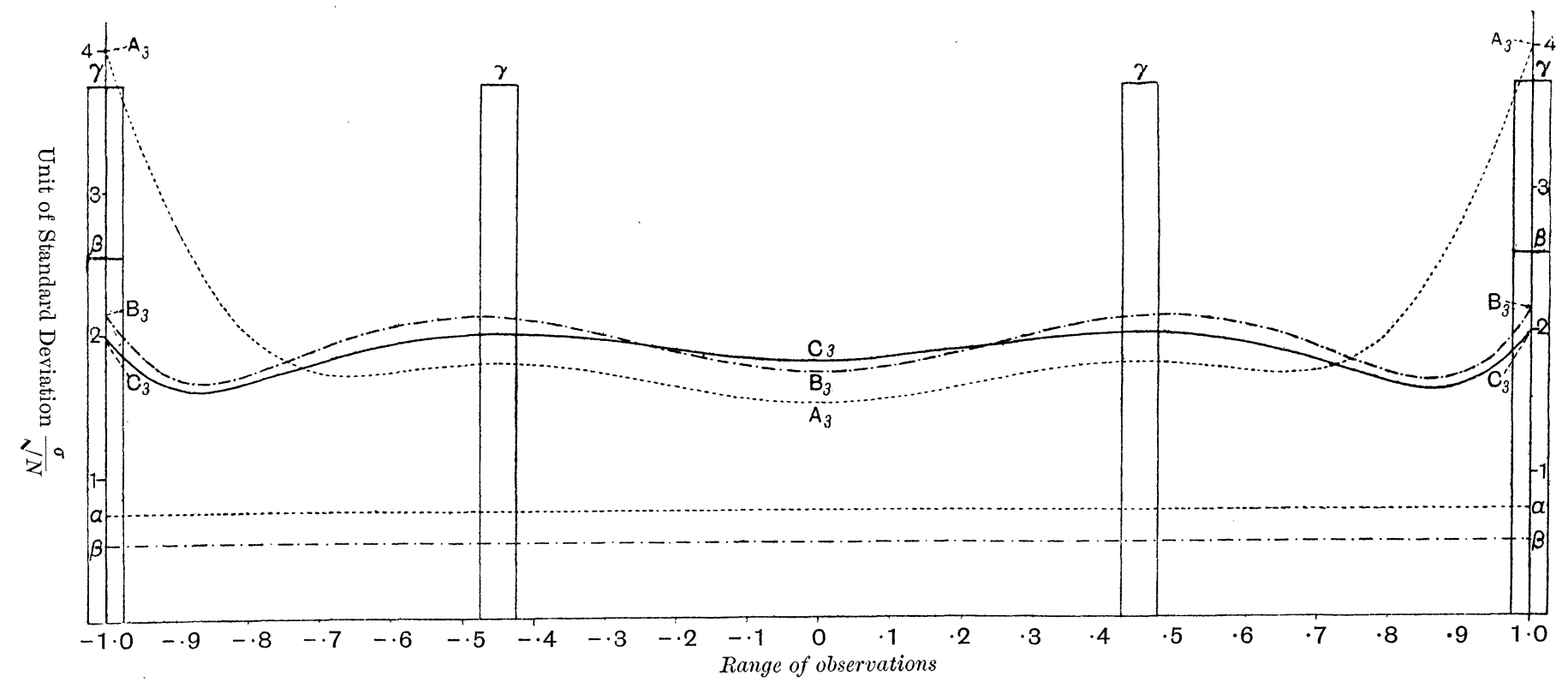

AA. Curve of Standard Deviations

aa. Distribution of Frequency of Observations

BB. Curve of Standard Deviations

Uniform distribution

$\beta \beta, \beta \beta$. Distribution of Frequency of Observations $\}$

-.-.- Uniform distribution with clusters

$\begin{aligned} & \beta \beta, \beta \beta \text { Distribution of Frequency of } \\ & \text { CC. Curve of Standard Deviations }\end{aligned}$

at the ends of range

$\gamma \gamma$. Distribution of Frequency of Observations

Equally big clusters of observations

at four points

Diagram 5. Function of Third Degree. 


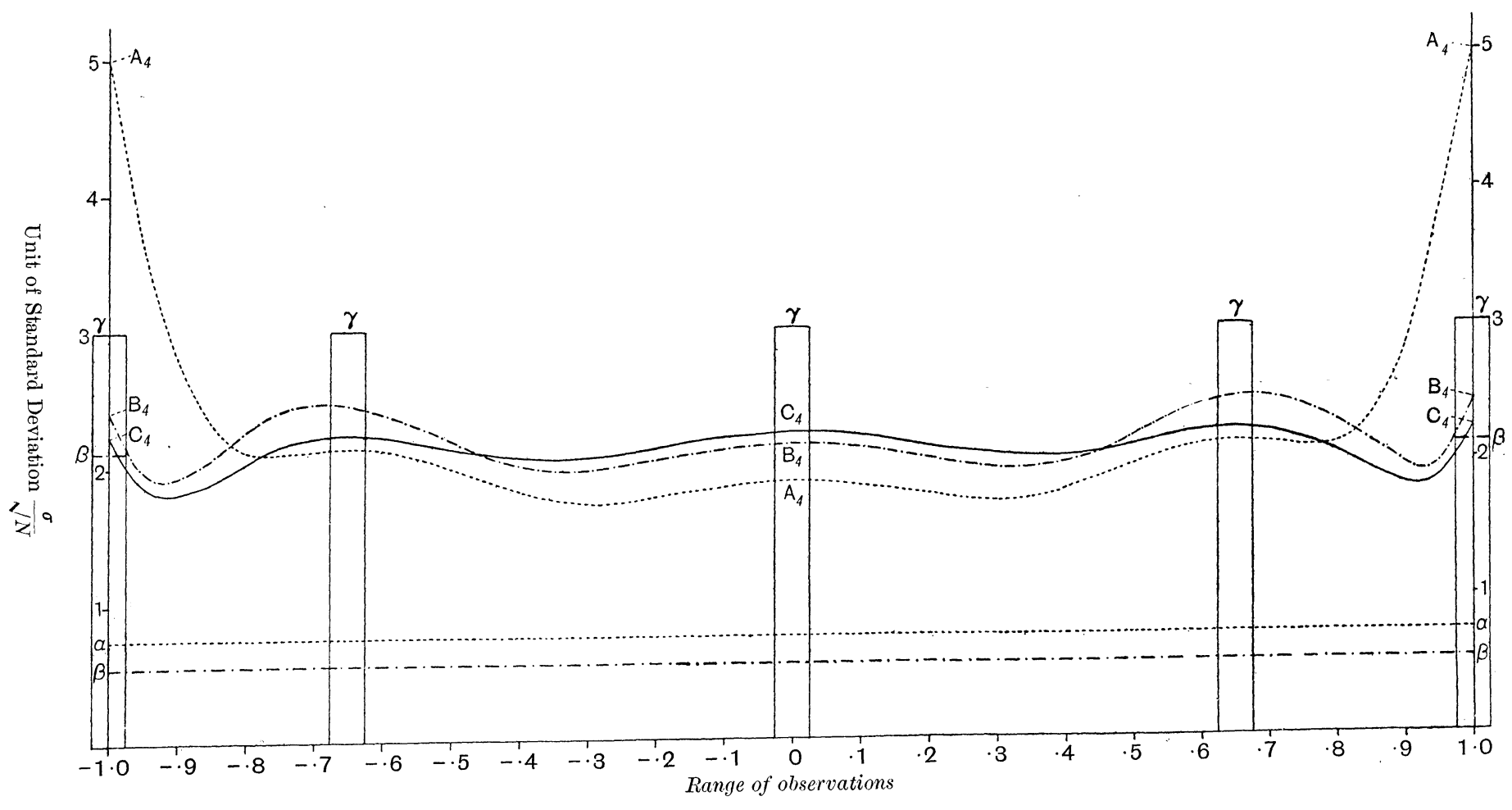

AA. Curve of Standard Deviations

aa. Distribution of Frequency of Observations

BB. Curve of Standard Deviations

\} ....... Uniform distribution at the ends of range

CC. Curve of Standard Deviations

Equally big clusters of observations

CC. Curve of Standard Deviations at five points

Diagram 6. Function of Fourth Degree. 


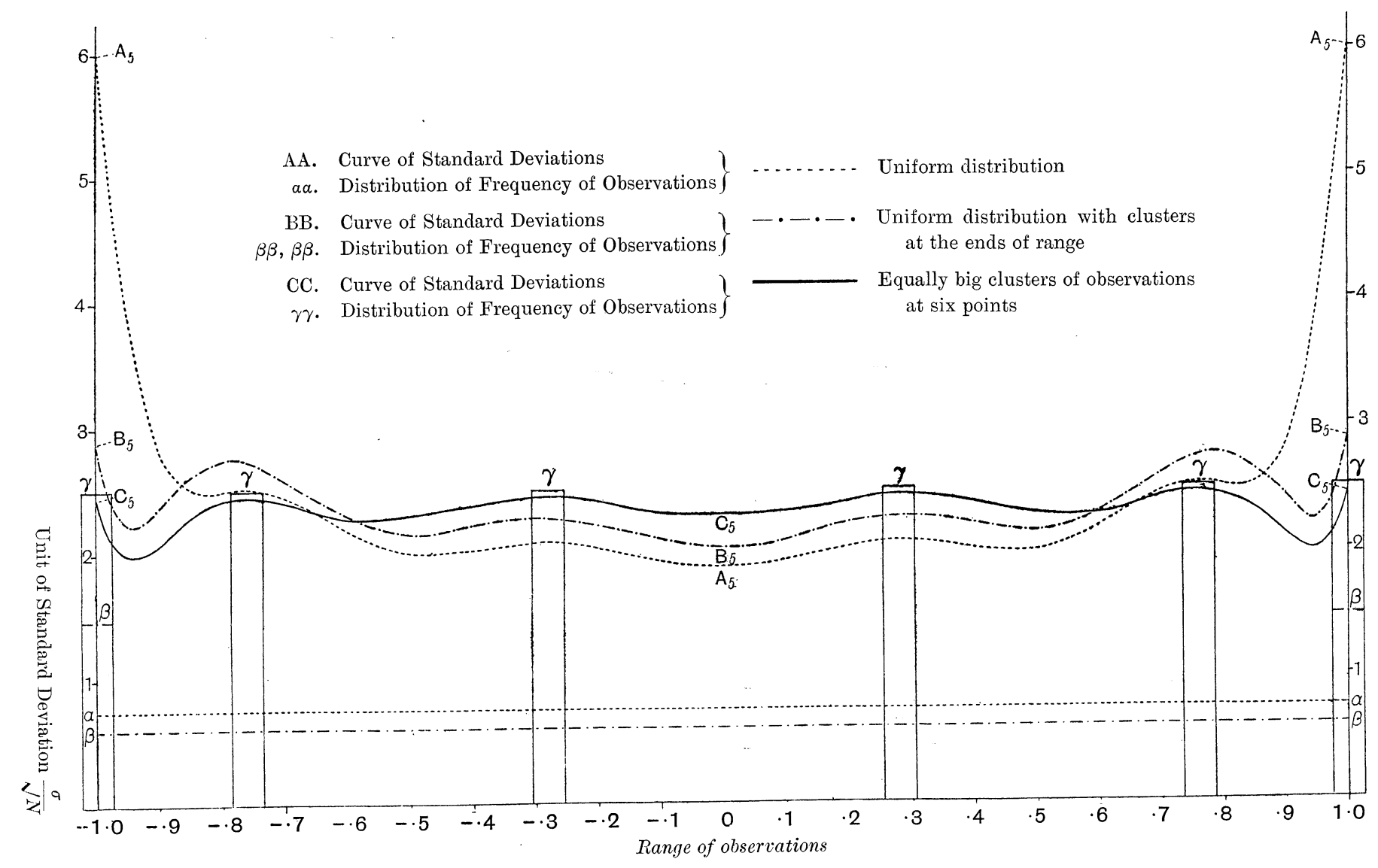

Diagram 7. Function of Fifth Degree. 


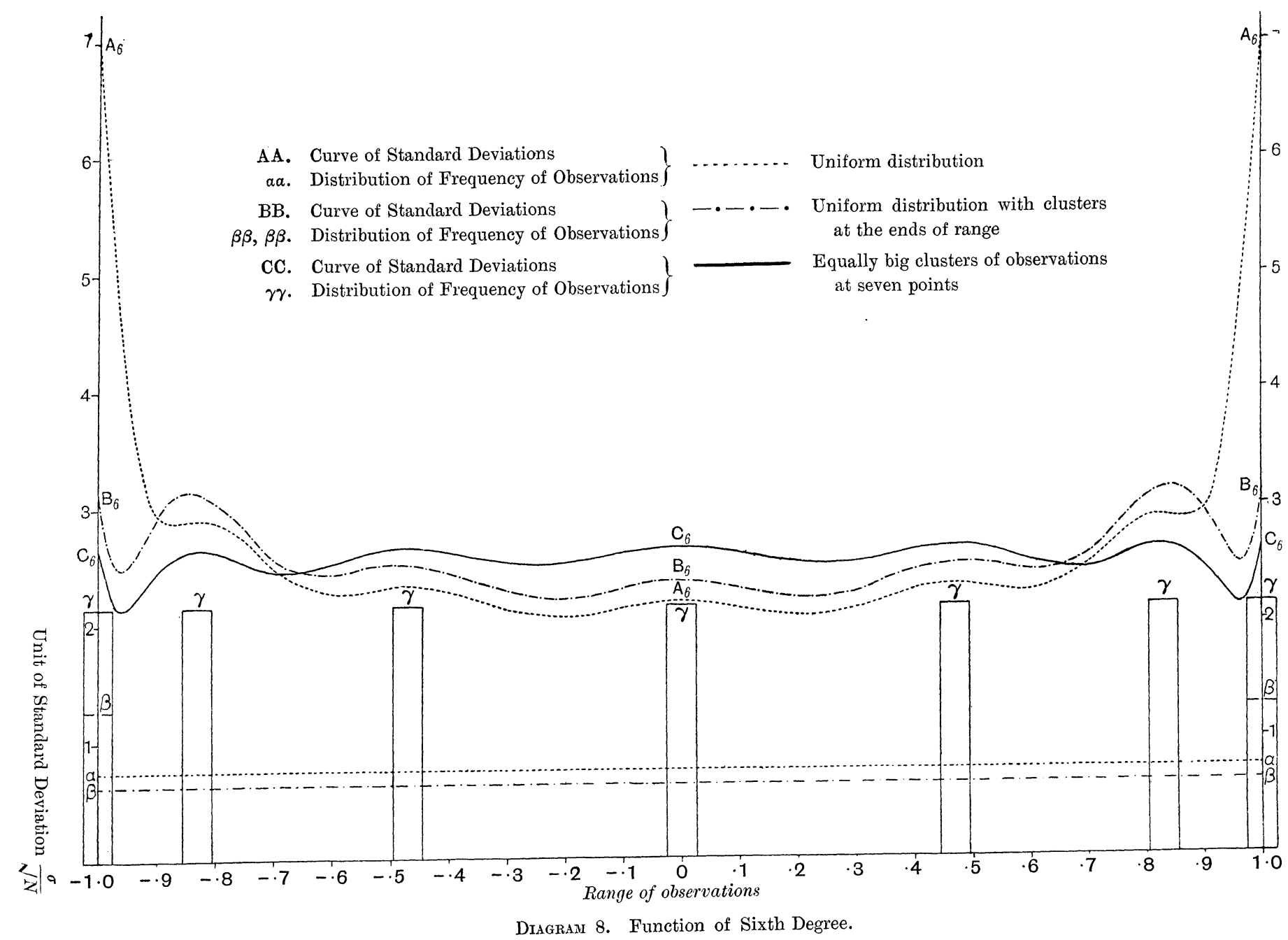




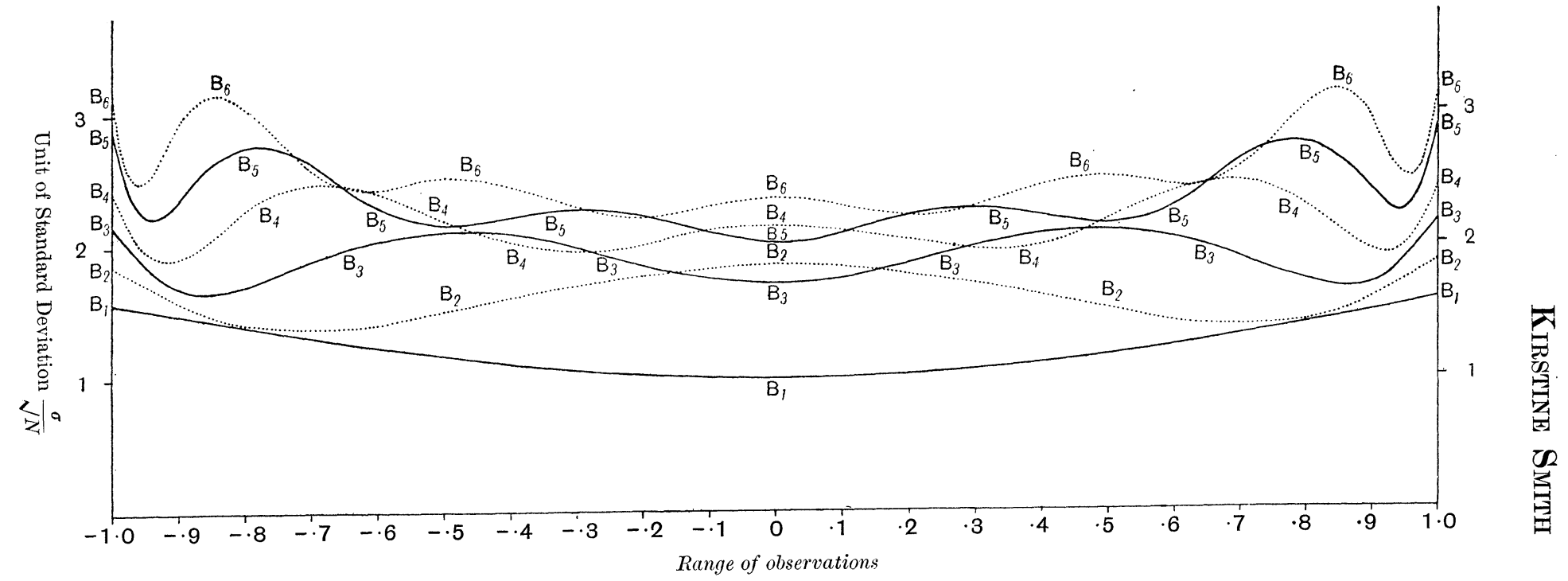

$\mathrm{B}_{1} \mathrm{~B}_{1}$. Function of First Degree

$\begin{array}{lll}\mathrm{B}_{2} \mathrm{~B}_{2} . & , & \text { Second , } \\ \mathrm{B}_{3} \mathrm{~B}_{3} . & , & \text { Third ,, } \\ \mathrm{B}_{4} \mathrm{~B}_{4} . & , & \text { Fourth , } \\ \mathrm{B}_{5} \mathrm{~B}_{5} . & , & \text { Fifth , } \\ \mathrm{B}_{6} \mathrm{~B}_{6} . & , & \text { Sixth , }\end{array}$

Diagram 9. Curves of Standard Deviations. Uniform Distribution with clusters at the ends of range. 
where $m_{p}=\frac{1}{N} \int x^{p} \frac{\psi(x)}{f(x)} d x, \psi(x) d x$ being the number of observations between $x$ and $x+d x$ and the integration being extended over the range of observations.

It is clear that if we have found a suitable curve of squared standard deviation for adjusted $y$ by taking a distribution $\phi(x)$ of observations with constant standard deviations a corresponding curve can be derived for observations with varying standard deviations by using the distribution

$$
\psi(x)=k \phi(x) \cdot f(x)
$$

As $\int k \phi(x) \cdot f(x) d x=N$ the constant $k$ must be

$$
k=\frac{N}{\int \phi(x) \cdot f(x) d x} .
$$

Hence we find

$$
m_{p}=\frac{\int x^{p} \cdot \phi(x) d x}{\int \phi(x) \cdot f(x) d x}=\frac{N \mu_{p}}{\int \phi(x) \cdot f(x) d x}
$$

where $\mu_{p}$ is the $p$ th moment coefficient for the distribution $\phi(x)$, and as

$$
\frac{m_{p}}{\mu_{p}}=\frac{N}{\int \phi(x) \cdot f(x) d x}=k
$$

for any $p$ the determinant may be written

$$
\left.\left|\begin{array}{cccccc}
\sigma_{y}^{2} \cdot \frac{N}{\sigma^{2}} k & 1 & x & x^{2} & \ldots \ldots & x^{n} \\
1 & 1 & \mu_{1} & \mu_{2} & \ldots \ldots & \mu_{n} \\
x & \mu_{1} & \mu_{2} & \mu_{3} & \ldots \ldots & \mu_{n+1} \\
x^{2} & \mu_{2} & \mu_{3} & \mu_{4} & \ldots \ldots & \mu_{n+2} \\
\vdots & \vdots & \vdots & \vdots & \vdots \\
x^{n} & \mu_{n} & \mu_{n+1} & \mu_{n+2} & \ldots \ldots & \mu_{2 n}
\end{array}\right|=0 \quad \ldots \ldots \ldots \ldots \ldots . . .66\right) .
$$

We thus find the same determinant as the distribution $\phi(x)$ would give for observations with constant error of observation except that the factor $k$ has come in, that is to say the expression for $\sigma_{y}^{2}$ has been multiplied by

$$
\frac{1}{k}=\frac{1}{N} \int \phi(x) \cdot f(x) d x
$$

The goodness of the distribution therefore will partly depend on the value of $\frac{1}{k}$, and because we have found $\phi(x)$ the best distribution for observations with constant standard deviation it does not follow that

$$
\psi(x)=k \phi(x) \cdot f(x)
$$

is the best distribution for observations with the standard deviation $\sigma \sqrt{f(x)}$. But the deriving of $\psi(x)$ from $\phi(x)$ is nevertheless useful as a means of simplifying the investigations and will be applied in the following special inquiries.

We shall consider two forms of $f(x)$ and try to find the best distributions for functions of the first and of the second degree. 
(a)

$$
f(x)=\left(1+\alpha x^{2}\right)^{2}, \text { where } \alpha>-1,
$$

for errors of observation increasing or decreasing in both directions from the middle of the range.

$$
f(x)=(1+\alpha x)^{2}, \text { where } 1>a \geqq 0,
$$

for error of observations increasing in one direction.

These two forms will roughly cover two distinct and important types of cases, such as occur in practice.

(2) When $f(x)=\left(1+a x^{2}\right)^{2}$ we find, according to (67),

and as (66) for $n=1$ gives

$$
\frac{1}{k}=1+2 \alpha \mu_{2}+\alpha^{2} \mu_{4},
$$

we have for a function of the first degree

$$
\sigma_{y}^{2} \cdot \frac{N}{\sigma^{2}} k=\frac{1}{\mu_{2}-\mu_{1}^{2}}\left\{\mu_{2}-2 \mu_{1} x+x^{2}\right\},
$$

$$
\sigma_{y}^{2}=\frac{\sigma^{2}}{N} \frac{1+2 \alpha \mu_{2}+\alpha^{2} \mu_{4}}{\mu_{2}-\mu_{1}^{2}}\left\{\mu_{2}-2 \mu_{1} x+x^{2}\right\}
$$

This curve has a minimum for $x=\mu_{1}$ and the maximum in the range is, if $\mu_{1}>0$, at $x=-1$, and if $\mu_{1}<0$, at $x=1$; it equals in both cases

$$
\frac{\sigma^{2}}{N}\left(1+2 \alpha \mu_{2}+\alpha^{2} \mu_{4}\right)\left\{1+\frac{\left(1+\left[\mu_{1}\right]\right)^{2}}{\mu_{2}-\mu_{1}^{2}}\right\}
$$

$\left[\mu_{1}\right]$ being the numerical value of $\mu_{1}$.

Now (69) is a minimum for $\mu_{1}=0$; we therefore ought to choose that value for $\mu_{1}$ and we then get, from (68),

$$
\sigma_{y}^{2}=\frac{\sigma^{2}}{N}\left(1+2 \alpha \mu_{2}+\alpha^{2} \mu_{4}\right)\left\{1+\frac{x^{2}}{\mu_{2}}\right\}
$$

$\mu_{2}$ and $\mu_{4}$ may vary between 0 and 1 independently of each other and are only bound by the conditions that

and

$$
\begin{gathered}
\mu_{4} \overline{\overline{<}} \mu_{2} \\
\beta_{2}=\frac{\mu_{4}}{\mu_{2}^{2}} \equiv 1 .
\end{gathered}
$$

For any set of values which satisfies these conditions we may determine a distribution consisting of $\frac{\gamma}{2} N$ observations at $x= \pm v$ and $(1-\gamma) N$ at $x=0$, since from any two such values we could determine

and

$$
\begin{aligned}
& v^{2}=\frac{\mu_{4}}{\mu_{2}} \bar{z} 1
\end{aligned}
$$

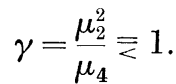

By introducing $v^{2}$ and $\gamma$ for $\mu_{2}$ and $\mu_{4}$ we get two quite independent variables and (70) then takes the form

$$
\sigma_{y}^{2}=\frac{\sigma^{2}}{N}\left(1+2 \alpha \gamma v^{2}+\alpha^{2} \gamma v^{4}\right)\left(1+\frac{x^{2}}{\gamma v^{2}}\right) .
$$


We now have to determine $\gamma$ and $v^{2}$ so that the maximum value $\sigma_{y}^{2}$ is as small as possible. We find

$$
\left[\frac{d \sigma_{y}^{2}}{d \gamma}\right]_{x=1}=\frac{\sigma^{2}}{N}\left(2 \alpha v^{2}+\alpha^{2} v^{4}-\frac{1}{\gamma^{2} v^{2}}\right)
$$

and

$$
\left[\frac{d \sigma_{y}^{2}}{d v^{2}}\right]_{x=1}=\frac{\sigma^{2}}{N}\left(2 \alpha \gamma+2 \alpha^{2} \gamma v^{2}+\alpha^{2}-\frac{1}{\gamma v^{4}}\right)
$$

Clearly

$$
\left[\frac{d \sigma_{y}^{2}}{d \gamma}\right]_{x=1}=0 \text { leads to } \gamma^{2}=\frac{1}{\alpha v^{4}\left(2+\alpha v^{2}\right)}
$$

Introducing this value into (72) we obtain

which is $>0$.

$$
\left[\frac{d \sigma_{y}^{2}}{d v^{2}}\right]_{x=1}=\frac{\sigma^{2}}{N} \cdot \alpha^{2}\left(1+\frac{1}{\sqrt{\alpha\left(2+\alpha v^{2}\right)}}\right),
$$

Hence the minimum for constant $v^{2}$ determined by

decreases with $v^{2}$.

$$
\left[\frac{d \sigma_{y}^{2}}{d \gamma}\right]_{x=1}=0
$$

But when $v^{2}$ decreases, $\gamma^{2}$, as given by (73), increases and the lowest value of $v^{2}$, for which it is real, is that determined by

$$
\gamma^{2}=\frac{1}{\alpha v^{4}\left(2+a v^{2}\right)}=1 \text {. }
$$

For $v^{2}$ smaller than this (73) gives $\gamma^{2}>1$, and as long as $\gamma^{2} \equiv 1$ we therefore have

$$
\left[\frac{d \sigma_{y}^{2}}{d \gamma}\right]_{x=1} \approx 0
$$

Hence the minimum of $\sigma_{y}^{x=1}$ is to be found for $\gamma^{2}=1$.

For this value (72) may be written as

$$
\left[\begin{array}{l}
d \sigma_{y}^{2} \\
d v^{2}
\end{array}\right]_{x=1}=\frac{\sigma^{2}}{N} \frac{1}{v^{4}}\left(1+\alpha v^{2}\right)\left(2 \alpha v^{4}+\alpha v^{2}-1\right)
$$

which is zero for

$$
v^{2}=-\frac{1}{4}+\sqrt{\frac{1+\frac{8}{\alpha}}{16}}
$$

and $>0$ for $v^{2}$ greater than this value.

When the $v^{2}$ found lies between 0 and 1 , that is when $\alpha>\frac{1}{3}$, we have thus found the minimum sought. When $\alpha \equiv \frac{1}{3}$, then $\left[\frac{d \sigma_{y}^{2}}{d v^{2}}\right]_{x=1}$ as given by $(74)$ is $<0$ and the minimum of $\sigma_{y}^{2}$ is found by giving $v^{2}$ its maximum value, that is 1 .

Returning to the variates $\mu_{2}$ and $\mu_{4}$ we see that in all cases

$$
\gamma^{2}=\frac{\mu_{2}^{2}}{\mu_{4}}=\frac{1}{\beta_{2}}=1,
$$


Kirstine Smith

from which it follows that no distribution of observations other than those arrived at consisting of two equally big groups can give $\mu_{1}, \mu_{2}$ and $\mu_{4}$ the values required.

We accordingly reach the result that: when observing a function of the first degree for which the standard deviation of the observations is $\sigma\left(1+\alpha x^{2}\right)$, symmetrical about the middle of the range, we get the best function for $\sigma_{y}^{2}$ by taking two equally big groups of observations, at the ends of the range if $\alpha \equiv \frac{1}{3}$ and at $v= \pm \frac{1}{2} \sqrt{\sqrt{1+\frac{8}{\alpha}}-1}$ if $a>\frac{1}{3}$.

(3) According to (70) the maximum of $\sigma_{y}^{2}$ for this distribution is

$$
\stackrel{x=1}{\sigma_{y}^{2}}=\frac{\sigma^{2}}{N}\left(1+\alpha v^{2}\right)^{2}\left(1+\frac{1}{v^{2}}\right),
$$

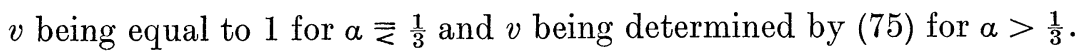

We shall next consider the distributions (i) for which $\phi(x)$ is constant from - 1 to 1 and (ii) for which $\phi(x)$ consists of $\frac{N}{2}$ observations uniformly distributed from -1 to 1 and $\frac{N}{2}$ into two clusters.

(i) For a uniform distribution from -1 to 1 we have $\mu_{2}=\frac{1}{3}, \mu_{4}=\frac{1}{5}$ and, according to (67),

$$
\frac{1}{k}=1+\frac{2}{3} \alpha+\frac{1}{5} \alpha^{2},
$$

the actual distribution is hence, as $\phi(x)=\frac{N}{2}$,

$$
\psi(x)=\frac{N}{2} \frac{\left(1+a x^{2}\right)^{2}}{1+\frac{2}{3} \alpha+\frac{1}{5} \alpha^{2}},
$$

and the maximum $\sigma_{y}^{2}$ as given by (70) for $x= \pm 1$,

$$
\stackrel{x= \pm 1}{\sigma_{\eta /}^{2}}=\frac{\sigma^{2}}{N}\left(1+\frac{2}{3} \alpha+\frac{1}{5} \alpha^{2}\right) \cdot 4 .
$$

(ii) When $\phi(x)=\frac{N}{4}$ with the additional clusters $\frac{N}{4}$ at $\pm u$ we have

$$
\mu_{2}=\frac{1}{6}+\frac{1}{2} u^{2} \text { and } \mu_{4}=\frac{1}{10}+\frac{1}{2} u^{4} .
$$

According to (70) the maximum $\sigma_{y}^{2}$ is then

$$
\stackrel{x= \pm 1}{\sigma_{y}^{2}}=\frac{\sigma^{2}}{N}\left[1+\alpha\left(\frac{1}{3}+u^{2}\right)+\alpha^{2}\left(\frac{1}{10}+\frac{1}{2} u^{2}\right)\right]\left(1+\frac{6}{3 u^{2}+1}\right) .
$$

We shall now determine $u$ so as to make this a minimum. We find that

requires

$$
\begin{aligned}
& \stackrel{x=1}{d \sigma_{y}^{2}} \\
& d u^{2}
\end{aligned}=\frac{\sigma^{2}}{N} \cdot \frac{1}{5}\left[5 a\left\{1+a\left(1+u^{2}\right)\right\}-\frac{2}{\left(3 u^{2}+1\right)^{2}}\left(45+7 \alpha^{2}\right)\right]=0
$$

the root $u^{2}$ of which is $>1$ for $\alpha<.5576$.

For $\alpha \equiv .5576$ we hence get the minimum $\sigma_{y}^{2}=1$ by taking the clusters at $u= \pm 1$ and for $\alpha>.5576$ at the places $\pm u$ determined by (76). 
Table III contains for a series of values of $a$ the values of $v,\left(1+\alpha v^{2}\right)$ and $u$ of the two distributions above and the maximum $\sigma_{y}$ for the three distributions.

TABLE III.

\begin{tabular}{|c|c|c|c|c|c|c|}
\hline$a$ & $v$ & $1+a v^{2}$ & $\begin{array}{l}\text { Maximum of } \\
\sigma_{y} \frac{\sqrt{ } N}{\sigma} \text { from } \\
\text { best distri- } \\
\text { bution }\end{array}$ & $\begin{array}{l}\text { Maximum of } \\
\sigma_{y} \frac{/ N}{\sigma} \text { from } \\
\text { distribution } \\
\text { for which } \\
\phi(x)=\frac{N}{2}\end{array}$ & $u$ & $\begin{array}{c}\text { Maximum of } \sigma_{y} \frac{\sqrt{ } N}{\sigma} \\
\text { from distribution } \\
\text { for which } \phi(x)=\frac{N}{4} \\
\text { and clusters of } \\
\frac{N}{4} \text { at } \pm u\end{array}$ \\
\hline 0 & 1.0000 & $1 \cdot 000$ & $1 \cdot 414$ & $2 \cdot 000$ & $1 \cdot 0000$ & 1.581 \\
\hline$\frac{1}{6}$ & $1 \cdot 0000$ & $1 \cdot 167$ & $1 \cdot 650$ & $2 \cdot 113$ & 1.0000 & $1 \cdot 760$ \\
\hline$\frac{1}{3}$ & $1 \cdot 0000$ & $1 \cdot 333$ & $1 \cdot 886$ & $2 \cdot 231$ & $1 \cdot 0000$ & 1.944 \\
\hline$\frac{0}{2}$ & $\cdot 8836$ & $1 \cdot 390$ & $2 \cdot 100$ & $2 \cdot 352$ & $1 \cdot 0000$ & $2 \cdot 131$ \\
\hline$\frac{\tilde{2}}{3}$ & $\cdot 8071$ & $1 \cdot 434$ & $2 \cdot 284$ & $2 \cdot 477$ & .9289 & $2 \cdot 316$ \\
\hline$\frac{3}{5}$ & .7510 & 1.470 & $2 \cdot 448$ & $2 \cdot 603$ & .8502 & $2 \cdot 483$ \\
\hline 1 & $\cdot 7071$ & $1 \cdot 500$ & $2 \cdot 598$ & $2 \cdot 733$ & .7797 & $2 \cdot 637$ \\
\hline 2 & .5559 & $1 \cdot 618$ & $3 \cdot 330$ & $3 \cdot 540$ & .5762 & $3 \cdot 438$ \\
\hline 3 & $\cdot 4782$ & $1 \cdot 686$ & $3 \cdot 908$ & $4 \cdot 382$ & .4925 & $4 \cdot 173$ \\
\hline 4 & $\cdot 4278$ & $1 \cdot 732$ & $4 \cdot 404$ & $5 \cdot 241$ & $\cdot 4612$ & $4 \cdot 899$ \\
\hline
\end{tabular}

The difference between the maxima from the two first distributions taken as a proportion of the maximum of the first decreases from 41 per cent. at $\alpha=0$ to the minimum 5 per cent. at $\alpha=1$, and then again increases to 19 per cent. at $\alpha=4$. For small $\alpha$, that is in practice $\alpha=0$, and again for $\alpha>3$, for which the difference is greater than 12 per cent., the third distribution may therefore be useful as giving a much smaller maximum value than the purely continuous distribution and at the same time offering some justification for the form of the function.

(4) We shall next, still assuming that $f(x)=\left(1+a x^{2}\right)^{2}$, consider the choice of observations for a function of the second degree.

According to (66) and (67) we find $\sigma_{y}^{2}=\frac{\sigma^{2}}{N} \cdot \frac{1}{k} \times$

$$
\left\{\frac{\mu_{2} \mu_{4}-\mu_{3}^{2}+2\left(\mu_{2} \mu_{3}-\mu_{1} \mu_{4}\right) x+\left(\mu_{4}-3 \mu_{2}^{2}+2 \mu_{1} \mu_{3}\right) x^{2}+2\left(\mu_{1} \mu_{2}-\mu_{3}\right) x^{3}+\left(\mu_{2}-\mu_{1}^{2}\right) x^{4}}{\mu_{2} \mu_{4}-\mu_{2}^{3}+2 \mu_{1} \mu_{2} \mu_{3}-\mu_{1}^{2} \mu_{4}-\mu_{3}^{2}}\right\}
$$

and

$$
\frac{1}{k}=1+2 \alpha \mu_{2}+\alpha^{2} \mu_{4}
$$

where the $\mu$ 's are the moment coefficients about $x=0$ of the distribution $\phi(x)$ which is connected with the actual distribution $\psi(x)$ by the relation

$$
\psi(x)=k \phi(x) . f(x) \text {. }
$$

From any distribution $\phi(x)$ which has $\mu_{1}$ and $\mu_{3} \gtrless 0$ we can form a symmetrical $\frac{1}{2}\{\phi(x)+\phi(-x)\}$ which has the same $\mu_{2}$ and $\mu_{4}$ as $\phi(x)$. We shall prove that the maximum $\sigma_{y}^{2}$ obtained from the symmetrical distribution is always lower than that obtained from the skew. 


\section{KiRstine SMith}

Let the factor in curled brackets in (77) be $F_{s}$ for a skew distribution $\phi(x)$ and $F_{0}$ for the corresponding symmetrical distribution.

We then have

$$
F_{0}=\frac{\mu_{2} \mu_{4}+\left(\mu_{4}-3 \mu_{2}^{2}\right) x^{2}+\mu_{2} x^{4}}{\mu_{2}\left(\mu_{4}-\mu_{2}^{2}\right)} .
$$

The condition for a maximum or minimum other than that at $x=0$ is

$$
\text { or } \begin{aligned}
3 \mu_{2}^{2}-\mu_{4} & >0, \\
\beta_{2} & <3,
\end{aligned}
$$

and as the denominator is positive we have in that case the maximum at $x=0$. It is thus clear that the maxima of $F_{0}$ between -1 and 1 must be either at $x=0$ or at $x= \pm 1$.

We shall show that

$$
\begin{gathered}
{\left[F_{s}\right]_{x=0}>\left[F_{0}\right]_{x=0},} \\
\text { and that either } \quad\left[F_{s}\right]_{x=1} \text { or }\left[F_{s}\right]_{x=-1}>\left[F_{0}\right]_{x= \pm 1} .
\end{gathered}
$$

According to what has been proved in Section I (4) the coefficient of $x^{4}$ in (77) is positive, the denominator of (77) is therefore positive and we have

$$
\left[\boldsymbol{F}_{s}-\boldsymbol{F}_{0}\right]_{x=0}=\frac{\left(\mu_{2} \mu_{3}-\mu_{1} \mu_{4}\right)^{2}}{\left(\mu_{4}-\mu_{2}^{2}\right)\left(\mu_{2} \mu_{4}-\mu_{2}^{3}+2 \mu_{1} \mu_{2} \mu_{3}-\mu_{1}^{2} \mu_{4}-\mu_{3}^{2}\right)}>0 .
$$

We shall next compare $F_{s}$ and $F_{0}$ for $x= \pm 1$.

Putting

$$
\left[F_{0}\right]_{x=1}=\frac{N}{D}
$$

we have

$$
\left[F_{s}\right]_{x=1}=\frac{N-\delta}{D-\epsilon},
$$

where

$$
\delta=\mu_{3}^{2}-2 \mu_{1} \mu_{3}+\mu_{1}^{2} \pm 2\left\{\mu_{3}\left(1-\mu_{2}\right)-\mu_{1}\left(\mu_{2}-\mu_{4}\right)\right\}
$$

and

For $\frac{\delta}{\epsilon}$ we find

$$
\epsilon=\mu_{3}^{2}-2 \mu_{1} \mu_{2} \mu_{3}+\mu_{1}^{2} \mu_{4} \text {. }
$$

$$
\frac{\delta}{\epsilon}=\frac{\left(\mu_{3}-\mu_{1}\right)^{2} \pm 2\left\{\mu_{3}\left(1-\mu_{2}\right)-\mu_{1}\left(\mu_{2}-\mu_{4}\right)\right\}}{\left(\mu_{3}-\mu_{1} \mu_{2}\right)^{2}+\mu_{1}^{2}\left(\mu_{4}-\mu_{2}^{2}\right)} .
$$

Looking first at the case $\frac{\mu_{1}}{\mu_{3}} \geqq 0$, we have

$$
\left(\mu_{3}-\mu_{1}\right)^{2}<\left(\mu_{3}-\mu_{1} \mu_{2}\right)^{2},
$$

and if we choose the value for which the other term of the numerator is $<0$,

$$
\frac{\delta}{\epsilon}<1 .
$$

When $\frac{\mu_{1}}{\mu_{3}}<0$ we see, from considering the form

$$
\underset{-\epsilon}{\delta_{3}}=1-\frac{\mu_{1}^{2}\left(1-\mu_{4}\right)-2 \mu_{1} \mu_{3}\left(1-\mu_{2}\right) \pm 2\left\{\mu_{3}\left(1-\mu_{2}\right)-\mu_{1}\left(\mu_{2}-\mu_{4}\right)\right\}}{\left(\mu_{3}-\mu_{1} \mu_{2}\right)^{2}+\mu_{1}^{2}\left(\mu_{4}-\mu_{2}^{2}\right)},
$$

that for either $x=1$ or $x=-1$

$$
\frac{\delta}{\epsilon}<1 \text {. }
$$


As $\epsilon>0$ we have hence for any $\mu_{1}$ and $\mu_{3}$, remembering that $\frac{N}{D}$ being a squared standard deviation multiplied by the number of observations is $\bar{\Sigma}$,

that is, for either $x=1$ or -1 ,

$$
\frac{N-\delta}{D-\epsilon}>\frac{N}{D}>\frac{\delta}{\epsilon},
$$

$$
F_{s}>F_{0} .
$$

We have thus proved that the maxima of $F_{0}$ are below those of $F_{s}$.

(5) Our problem is hence reduced to finding the best curve among those represented by

$$
\sigma_{y}^{2}=\frac{\sigma^{2}}{N} \frac{\left(1+2 a \mu_{2}+\alpha^{2} \mu_{4}\right)}{\mu_{2}\left(\mu_{4}-\mu_{2}^{2}\right)}\left\{\mu_{2} \mu_{4}+\left(\mu_{4}-3 \mu_{2}^{2}\right) x^{2}+\mu_{2} x^{4}\right\}
$$

As was stated in (2) of this section we get all sets of possible values for $\mu_{2}$ and $\mu_{4}$ from three groups of observations symmetrical about $x=0$, and we may therefore limit our search of the best distribution to these.

Let the observations be $\frac{\gamma}{2} N$ at $x= \pm v$, at $(1-\gamma) N$ at $x=0$. The interpolation formula of Lagrange gives, when $\bar{y}_{p}$ represents the mean of the observations at $x=p$,

from which we find

$$
y=\frac{x^{2}-v^{2}}{-v^{2}} \bar{y}_{0}+\frac{x(x-v)}{2 v^{2}} \bar{y}_{-v}+\frac{x(x+v)}{2 v^{2}} \bar{y}_{v},
$$

$$
\sigma_{y}^{2}=\frac{\sigma^{2}}{N} \cdot \frac{1}{v^{4}}\left\{\frac{\left(x^{2}-v^{2}\right)^{2}}{1-\gamma}+\frac{x^{2}\left(x^{2}+v^{2}\right)\left(1+\alpha v^{2}\right)^{2}}{\gamma}\right\}
$$

It is obvious that if for a certain distribution we have

$$
\begin{gathered}
x=0 \quad x^{2}=1 \\
\sigma_{y}^{2}>\sigma_{y}^{2}
\end{gathered}
$$

we can get a better distribution by taking more observations at 0 . If on the other hand

$$
\begin{gathered}
x=0 \quad x^{2}=1 \\
\sigma_{y}^{2}<\sigma_{y}^{2}, \\
x^{2}=1
\end{gathered}
$$

the curve cannot be the best unless $\sigma_{y}^{2}$ is a minimum for the present values of $v$ and $\gamma$. From (79) we find

$$
\left[\frac{d \sigma_{y}^{2}}{d \gamma}\right]_{x \quad 1}=\frac{\sigma^{2}}{N} \cdot \frac{1}{v^{4}}\left\{\frac{\left(1-v^{2}\right)^{2}}{(1-\gamma)^{2}}-\frac{\left(1+v^{2}\right)\left(1+\alpha v^{2}\right)^{2}}{\gamma^{2}}\right\}
$$

and

$$
\left[\frac{d \sigma_{y}^{2}}{d v^{2}}\right]_{x^{2}=1}=\frac{\sigma^{2}}{N} \cdot \frac{1}{v^{6}}\left\{-\frac{2\left(1-v^{2}\right)}{1-\gamma}-\frac{\left(2+v^{2}-\alpha v^{4}\right)\left(1+a v^{2}\right)}{\gamma}\right\},
$$

from which we obtain the conditions for maximum or minimum

and

$$
\begin{gathered}
v^{2}=\frac{1 \pm 2 \sqrt{\alpha}}{a} \\
\frac{\gamma}{1-\gamma}=\frac{2 \sqrt{\alpha}(1 \pm \sqrt{\alpha})^{2}}{a \mp 2 \sqrt{\alpha}-1}
\end{gathered}
$$




\section{Kinstine SMith}

The lower sign requires $3-2 \sqrt{2} \bar{\Sigma} \alpha \bar{\Sigma}$ and the upper sign $a>3+2 \sqrt{2}$ to make $0 ₹ v^{2} ₹ 1$. The case $\alpha<\frac{1}{4}$ has no interest, as we have seen that when $\alpha<\frac{1}{3}$ extrapolation is not even for a linear function advantageous. We have therefore seen that for $a<3+2 \sqrt{2} * \frac{x^{2}=1}{\sigma_{y}^{2}}$ has no minimum and we have thus proved that $\begin{aligned} x=0 & x^{2}=1\end{aligned}$ the best distribution requires $\sigma_{y}^{2}=\sigma_{y}^{2}$, that is

or

$$
\begin{aligned}
\frac{2 v^{2}-1}{1-\gamma} & =\frac{\left(1+v^{2}\right)\left(1+\alpha v^{2}\right)^{2}}{\gamma} \\
\frac{1}{1-\gamma} & =1+\frac{\left(1+v^{2}\right)\left(1+\alpha v^{2}\right)^{2}}{2 v^{2}-1}
\end{aligned}
$$

The maximum of the curve is

$$
\stackrel{x=0}{\sigma_{y}^{2}}=\frac{\sigma^{2}}{N} \cdot \frac{1}{1-\gamma} .
$$

To find the minimum of this value we differentiate (81) and get

which is zero for

$$
\left[\frac{d \sigma_{\eta}^{2}}{d v^{2}}\right]_{x=0}=\frac{1+a v^{2}}{\left(2 v^{2}-1\right)^{2}}\left\{4 a v^{4}-a v^{2}-2 \alpha-3\right\},
$$

$$
v^{2}=\frac{1}{8}\left(1+\sqrt{33+\frac{48}{a}}\right)
$$

and positive for greater $v^{2}$, so that we have found a minimum.

For $\alpha=3$ we find from (82) $v^{2}=1$, hence for $\alpha \bar{₹} 3$ we have to choose $v^{2}=1$, from which, according to (81), follows

$$
\frac{1}{1-\gamma}=1+2(1+\alpha)^{2} \text { or } \gamma=\frac{2(1+\alpha)^{2}}{1+2(1+\alpha)^{2}} .
$$

When $3+2 \sqrt{2}>a>3, v^{2}=\frac{1}{8}\left(1+\sqrt{33+\frac{48}{a}}\right)$ is $<1$, and for the corresponding $\gamma$ we have

$$
\frac{\frac{1}{2} \gamma}{\left(1+\alpha v^{2}\right)^{2}}=\frac{1+v^{2}}{2\left(2 v^{2}-1\right)}=\frac{5 \alpha+4+\sqrt{\alpha(33 \alpha+48)}}{8(\alpha+2)}
$$

Returning to the $\phi(x)$ distribution, which is found from this distribution by dividing the frequencies by $k \cdot\left(1+\alpha x^{2}\right)^{2}$, we therefore find, when $\frac{\epsilon}{2} N$ is the number of observations at $x= \pm v$ and $(1-\epsilon) N$, that, at $x=0$,

$$
\frac{\overline{2}}{1-\epsilon}=\frac{1+v^{2}}{2\left(2 v^{2}-1\right)}
$$

* A further examination shows that for $a>3+2 \sqrt{2} \sigma_{y}^{x^{2}=1}$ has a minimum but this is smaller than \[ \begin{array}{c}x^{2}=1 \quad x=0 \\ x=0 \\ \sigma_{y}^{2}\end{array} \text { when } a<6 \cdot 7 \text {. Up to this value we therefore have } \sigma_{y}^{2}=\sigma_{y}^{2} \text { for the best curves. For } a>6 \cdot 7 \text { the } \] minimum of $\sigma_{y}^{2}$ determines the best distribution.

Biometrika XII 
or

$$
\epsilon=\frac{1+v^{2}}{3 v^{2}}
$$

Hence

and

$$
\left.\begin{array}{l}
\mu_{2}=\frac{1}{3}\left(1+v^{2}\right) \\
\mu_{4}=\frac{v^{2}}{3}\left(1+v^{2}\right)
\end{array}\right\}
$$

For $\alpha \equiv 3$ we have found $v^{2}=1$ which according to (84) involves $\mu_{2}=\mu_{4}$, so that only the distribution above consisting of three groups can realise the requisite conditions.

When $\alpha>3$ we have $v<1$ and therefore $\mu_{4}<\mu_{2}$, so that it must be possible to satisfy the equation (84) by a continuous distribution of observations. However $v^{2}$ is decreasing so slowly for increasing a that practically the distribution determined by (84) cannot differ much from three groups of observations.

Our results are accordingly that for a function of the second degree, of which the standard deviation of the observations is $\sigma\left(1+a x^{2}\right)$, we get the best function for $\sigma_{y}^{2}$ when $a \equiv 3$ by taking three groups of observations at the middle and the ends of the range, each group proportional to the squared standard deviation at the place, and when $3+2 \sqrt{2}>\alpha>3$ by taking three groups of observations determined by (82) and (83).

(6) From (78) we find

$$
\stackrel{x=0}{\sigma_{y}^{2}}=\frac{\sigma^{2}}{N}\left(1+2 \alpha \mu_{2}+a^{2} \mu_{4}\right) \frac{\mu_{4}}{\mu_{4}-\mu_{2}^{2}},
$$

which, when $\mu_{2}$ and $\mu_{4}$ are found in accordance with (82) and (84), determines the maximum $\sigma_{y}$ arrived at from our special three groups of observations. Besides the numerical evaluation of this standard deviation, we give in Table IV below the maximum of $\sigma_{y}$ obtained from a distribution for which $\phi(x)$ is constant from -1 to 1 , that is, since, according to (67),

$$
\frac{1}{k}=\left(1+\frac{2}{3} \alpha+\frac{\alpha^{2}}{5}\right)
$$

the distribution

$$
\psi(x)=\frac{\left(1+\alpha x^{2}\right)^{2}}{1+\frac{2}{3} \alpha+\frac{a^{2}}{5}} \cdot \frac{N}{2} .
$$

That maximum is determined by

$$
\sigma_{y}^{2}=\frac{\sigma^{2}}{N}\left(1+\frac{2}{3} \alpha+\frac{\alpha^{2}}{5}\right) \cdot 9
$$

$\frac{\sigma^{2}}{N} .9$ being the maximum $\sigma_{y}^{2}$ obtained from a rectangular distribution of observations with the standard deviation $\sigma$.

The last column of the same table gives the maximum $\sigma_{y}$ arrived at when $\phi(x)$ is the rectangular distribution with clusters at -1 and 1 for which $\begin{gathered}x=0 \\ \sigma_{y}^{2}=x^{2}=1 \\ \sigma_{y}^{2}\end{gathered}$. For this distribution consisting of $\cdot 22026 N$ observations at +1 and at -1 and . $5595 N=2 c N$ uniformly distributed from -1 to 1 , we have found as given in 
Table II (p. 50) the maximum $\frac{\sigma}{\sqrt{ } N} \cdot 1 \cdot 862$. Hence when $\mu_{2}$ and $\mu_{4}$ are the moment coefficient of this $\phi(x)$ the maximum is found from

$$
\sigma_{y}^{2}=\frac{\sigma^{2}}{N}\left(1+2 \alpha \mu_{2}+\alpha^{2} \mu_{4}\right) \cdot 1 \cdot 862 .
$$

We find $\mu_{2}=\cdot 6270, \mu_{4}=\cdot 5524$ and $\frac{1}{k}=1+1 \cdot 2540 \alpha+\cdot 5524 \alpha^{2}$.

The actual distribution is hence

together with the clusters

$$
\psi(x)=\frac{\cdot 27975\left(1+\alpha x^{2}\right)^{2}}{1+1 \cdot 2540 \alpha+\cdot 5524 \alpha^{2}} \cdot N
$$

at -1 and 1.

$$
\frac{\cdot 22026(1+\alpha)^{2}}{1+1 \cdot 2540 \alpha+\cdot 5524 \alpha^{2}} \cdot N
$$

\begin{tabular}{|c|c|c|c|}
\hline$\alpha$ & $\begin{array}{l}\text { Maximum of } \\
\sigma_{y} \frac{\sqrt{ } N}{\sigma} \text { for } \\
\text { the best } \\
\text { distribution }\end{array}$ & $\begin{array}{c}\text { Maximum of } \sigma_{y} \frac{\sqrt{ } N}{\sigma} \\
\text { for distribution } \\
\text { with } \phi(x)=\frac{N}{2}\end{array}$ & $\begin{array}{c}\text { Maximum of } \sigma_{y} \frac{\sqrt{ } N}{\sigma} \text { for } \\
\text { distribution with } \\
\phi(x)=c \text { and clusters } \\
\text { at } \pm 1\end{array}$ \\
\hline $\begin{array}{l}0 \\
1 \\
2 \\
3 \\
4 \\
5\end{array}$ & $\begin{array}{l}1 \cdot 732 \\
3 \cdot 000 \\
4 \cdot 359 \\
5 \cdot 745 \\
7 \cdot 135 \\
8 \cdot 522\end{array}$ & $\begin{array}{l}3 \cdot 000 \\
4 \cdot 099 \\
5 \cdot 310 \\
6 \cdot 573 \\
7 \cdot 861 \\
9 \cdot 165\end{array}$ & $\begin{array}{l}1 \cdot 862 \\
3 \cdot 120 \\
4 \cdot 453 \\
5 \cdot 810 \\
7 \cdot 178 \\
8 \cdot 551\end{array}$ \\
\hline
\end{tabular}

TABLE IV.

The difference between the first and second maxima taken as a proportion of the first varies from 79 per cent. at $\alpha=0$ to 8 per cent. at $\alpha=5$, while the difference between the first and the third maxima varies from 8 per cent. at $\alpha=0$ to $0 \cdot 4$ per cent. at $a=5$. The continuous distribution with clusters is therefore especially useful for smaller $\alpha$.

For $\alpha=4$ we find from (82) $v=.9816$ and for $\alpha=5, v=\cdot 9700$, both of these values of $v$ are so close to 1 that if instead of using them we take the observations at 1 and -1 and let the numbers of the three groups of observations be proportional to the squared standard deviations we get the maxima $7 \cdot 141$ and 8.544 which only differ quite insignificantly from the corresponding values of Table IV.

(7) For a function of the first degree, of which the standard deviation of the

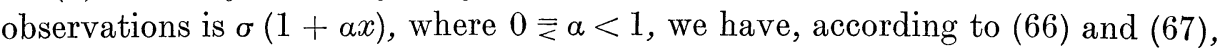

$$
\sigma_{y}^{2}=\frac{\sigma^{2}}{N} \frac{1+2 \alpha \mu_{1}+\alpha^{2} \mu_{2}}{\mu_{2}-\mu_{1}^{2}}\left\{\mu_{2}-2 \mu_{1} x+x^{2}\right\}
$$

For $\mu_{1}=-c^{2}$ the maximum of this function is at $x=1$, and for $\mu_{1}=c^{2}$ at -1 . As the maximum of $\left(\mu_{2}-2 \mu_{1} x+x^{2}\right)$ has the same value in both cases it is clear 
that the negative $\mu_{1}$ gives the lower maximum for $\sigma_{y}^{2}$. We therefore only have to find the conditions for $\left[\sigma_{y}^{2}\right]_{x=1}$ being a minimum when $\mu_{1}<0$.

We have

$$
\stackrel{x=1}{\sigma_{y}^{2}}=\frac{\sigma^{2}}{N} \frac{\left[\left(1+\alpha \mu_{1}\right)^{2}+\alpha^{2}\left(\mu_{2}-\mu_{1}^{2}\right)\right]}{\mu_{2}-\mu_{1}^{2}}\left\{\mu_{2}-\mu_{1}^{2}+\left(1-\mu_{1}\right)^{2}\right\}
$$

and differentiating with regard to $\mu_{2}$,

$$
\left[\frac{d \sigma_{\eta}^{2}}{d \mu_{2}}\right]_{x=1}=\frac{\sigma^{2}}{N} \cdot \frac{\alpha^{2}\left(\mu_{2}-\mu_{1}^{2}\right)^{2}-\left(1-\mu_{1}\right)^{2}\left(1+\alpha \mu_{1}\right)^{2}}{\left(\mu_{2}-\mu_{1}^{2}\right)^{2}} .
$$

As $\alpha<1$, we have $\left(1-\mu_{1}\right)\left(1+\alpha \mu_{1}\right)>0$ and

$$
\alpha\left(\mu_{2}-\mu_{1}^{2}\right)-\left(1-\mu_{1}\right)\left(1+\alpha \mu_{1}\right)=\left(\alpha \mu_{2}-1\right)+\mu_{1}(1-\alpha)<0,
$$

from which it follows that

for any $\mu_{1} \bar{\Sigma} 0$.

$$
\left[\frac{d \sigma_{y}^{2}}{d \mu_{2}}\right]_{x=1}<0
$$

The greatest value $\mu_{2}$ can take for our range -1 to +1 is 1 , the minimum of

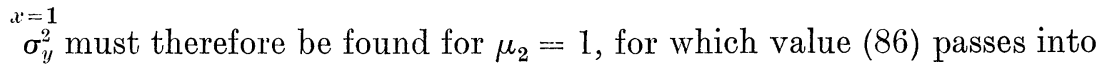

$$
\left[\sigma_{y}^{2}\right]_{x=1}=\frac{\sigma^{2}}{N} 2\left\{2 \alpha+\frac{(1-\alpha)^{2}}{1+\mu_{1}}\right\}
$$

which, since $\mu_{1} \equiv 0$, is a minimum and equals $\frac{\sigma^{2}}{N} \cdot 2\left(1+\alpha^{2}\right)$ when $\mu_{1}=0$.

The $\phi(x)$ distribution ought accordingly to consist of two equally big groups at the ends of the range and the actual distribution to be chosen for a function of the first degree, the standard deviation of which is a linear function of the variable, should be two groups at the ends of the working range with numbers proportional to the squared standard deviations at these places.

(8) For a continuous distribution from -1 to 1 with frequencies proportional to the squared standard deviations we have

and the maximum

$$
\begin{aligned}
& \mu_{1}=0 \text { and } \mu_{2}=\frac{1}{3}, \\
& x=1 \\
& \sigma_{y}^{2}=\frac{\sigma^{2}}{N}\left(1+\frac{\alpha^{2}}{3}\right) 4,
\end{aligned}
$$

the actual distribution is $\quad \psi(x)=\frac{(1+\alpha x)^{2}}{1+\frac{a^{2}}{3}} \cdot \frac{N}{2}$.

Table $\mathrm{V}$ contains besides the maxima of $\sigma_{y}$ from these two distributions those obtained from a distribution for which $\phi(x)$ is constant with two additional clusters at -1 and 1 each consisting of $\frac{N}{4}$ of the observations.

The actual distribution is, since

$$
\begin{gathered}
\mu_{2}=\frac{1}{2}+\frac{1}{6}=\frac{2}{3}, \\
\psi(x)=\frac{(1+\alpha x)^{2}}{1+\frac{2}{3} \alpha^{2}} \cdot \frac{N}{4},
\end{gathered}
$$


with

$$
\frac{(1-\alpha)^{2}}{1+\frac{2}{3} \alpha^{2}} \cdot \frac{N}{4} \text { observations at }-1
$$

and

$$
\frac{(1+\alpha)^{2}}{1+\frac{2}{3} \alpha^{2}} \cdot \frac{N}{4} \text { at }+1 \text { in addition. }
$$

The maximum of $\sigma_{y}^{2}$ is

$$
\frac{\sigma^{2}}{N}\left(1+\frac{2}{3} \alpha^{2}\right) \frac{5}{2}
$$

\begin{tabular}{|c|c|c|c|}
\hline$a$ & $\begin{array}{l}\text { Maximum of } \\
\sigma_{y} \frac{\sqrt{ } N}{\sigma} \text { for } \\
\text { best distri- } \\
\text { bution }\end{array}$ & $\begin{array}{c}\text { Maximum of } \sigma_{y} \frac{\sqrt{ } N}{\sigma} \\
\text { for distribution } \\
\text { with } \phi(x)=\frac{N}{2}\end{array}$ & $\begin{array}{c}\text { Maximum of } \sigma_{y} \frac{\sqrt{ } N}{\sigma} \text { for } \\
\text { distribution with } \\
\phi(x)=\frac{N}{4} \text { and clusters } \\
\text { at } \pm 1\end{array}$ \\
\hline $\begin{array}{l}\cdot 0 \\
.1 \\
\cdot 2 \\
\cdot 3 \\
.4 \\
.5 \\
.6 \\
\cdot 7 \\
\cdot 8 \\
.9\end{array}$ & $\begin{array}{l}1 \cdot 414 \\
1 \cdot 421 \\
1 \cdot 442 \\
1 \cdot 477 \\
1 \cdot 523 \\
1 \cdot 581 \\
1 \cdot 649 \\
1 \cdot 726 \\
1 \cdot 811 \\
1.903\end{array}$ & $\begin{array}{l}2 \cdot 000 \\
2 \cdot 003 \\
2 \cdot 013 \\
2 \cdot 030 \\
2 \cdot 053 \\
2 \cdot 082 \\
2 \cdot 117 \\
2 \cdot 157 \\
2 \cdot 203 \\
2 \cdot 254\end{array}$ & $\begin{array}{l}1 \cdot 581 \\
1 \cdot 587 \\
1 \cdot 602 \\
1 \cdot 628 \\
1 \cdot 663 \\
1 \cdot 708 \\
1 \cdot 761 \\
1 \cdot 821 \\
1 \cdot 889 \\
1 \cdot 962\end{array}$ \\
\hline
\end{tabular}

TABLE V.

(9) For a function of the second degree we found in (5) that when the standard deviation of the observations was $s_{y}=\sigma\left(1+\alpha x^{2}\right)$ and $\alpha \equiv 3$ it was advantageous to use the whole working range of observations, much more must this be the case when $s_{y}=\sigma(1+\alpha x)$ and $0 \equiv \alpha<1$. We shall therefore try to find the three best groups of observations taken at $-1, v$, and 1 , supposing $v$ unknown. We do not venture to assert that another form of distribution might not lead to a curve of standard deviation with lower maximum, but the solution of the general problem would involve a more elaborate investigation into the possible variations of $\mu_{1}, \mu_{2}$, $\mu_{3}$ and $\mu_{4}$ for distributions with limited range than seems desirable in this connection. We shall further limit our problem by assuming that the best distribution will be found among those which make $\begin{gathered}x=1 \\ \sigma_{y}^{2}\end{gathered}=\sigma_{y}^{2}$ and both also equal to a maximum situated between $x=-1$ and $x=1$. This would obviously be right if the maximum were found at $x=v$; this in fact is not the case, but still the maximum value is likely to be chiefly determined by the number of observations at $x=v$ and there is therefore every reason to believe that our assumption is justifiable.

Let there be $N \delta$ observations at $-1, N \cdot \gamma$ at 1 and $N(1-\delta-\gamma)$ at $v$. The interpolation formula of Lagrange then gives

$$
y=\frac{(x-v)(x-1)}{(1+v) \cdot 2} \bar{y}_{-1}+\frac{(x-v)(x+1)}{(1-v) \cdot 2} \bar{y}_{1}+\frac{x^{2}-1}{v^{2}-1} \bar{y}_{v},
$$


from which we find

$$
\begin{aligned}
& \sigma_{y}^{2}=\frac{\sigma^{2}}{N}\left\{\frac{(x-v)^{2}(x-1)^{2}}{4(1+v)^{2}} \cdot \frac{(1-\alpha)^{2}}{\delta}+\frac{(x-v)^{2}(x+1)^{2}}{4(1-v)^{2}} \cdot \frac{(1+\alpha)^{2}}{\gamma}\right. \\
& \text { The condition for } \left.\quad+\frac{\left(x^{2}-1\right)^{2}}{\left(v^{2}-1\right)^{2}} \cdot \frac{(1+\alpha v)^{2}}{1-\delta-\gamma}\right\} . \\
& \begin{array}{c}
x=1 \\
\sigma_{y}^{2}=\sigma_{y}^{2}=-1
\end{array}
\end{aligned}
$$

is

$$
\frac{(1+\alpha)^{2}}{\gamma}=\frac{(1-\alpha)^{2}}{\delta}
$$

Eliminating $\delta$ we obtain for $\sigma_{y}^{2}-\sigma_{y}^{2}$ the value

$$
\begin{aligned}
& \sigma_{\nu}^{2}-\sigma_{y}^{2}=1 \\
& \bar{N} \cdot \frac{\sigma^{2}}{N} \cdot \frac{(1+\alpha)^{2}\left(x^{2}-1\right)}{\left(v^{2}-1\right)^{2}}\left\{\frac{(1+\alpha v)^{2}\left(x^{2}-1\right)}{(1+\alpha)^{2}-2 \gamma\left(1+\alpha^{2}\right)}\right. \\
&+\frac{1}{2 \gamma} {\left.\left[\left(1+v^{2}\right) x^{2}+2 v\left(1-v^{2}\right) x+2-5 v^{2}+v^{4}\right]\right\} }
\end{aligned}
$$

or

$$
\begin{aligned}
\sigma_{y}^{2}-\sigma_{\nu}^{2=1}=\frac{\sigma^{2}}{\bar{N}} & \frac{(1+\alpha)^{2}\left(x^{2}-1\right)}{\left(v^{2}-1\right)^{2}\left[(1+\alpha)^{2}-2 \gamma\left(1+\alpha^{2}\right)\right]}\left\{\left[(1+\alpha)^{2}\left(1+v^{2}\right)-2 \gamma(\alpha-v)^{2}\right] x^{2}\right. \\
& +2 v\left(1-v^{2}\right)\left[(1+\alpha)^{2}-2 \gamma\left(1+\alpha^{2}\right)\right] x+(1+\alpha)^{2}\left(2-5 v^{2}+v^{4}\right) \\
& \left.-2 \gamma\left[\left(1+\alpha^{2}\right)\left(2-5 v^{2}+v^{4}\right)+(1+\alpha v)^{2}\right]\right\} \ldots \ldots \ldots \ldots \ldots \ldots \ldots \ldots \ldots \ldots \ldots \ldots \ldots \ldots
\end{aligned}
$$

Our assumption that the maximum $\sigma_{y}^{2}$ shall be equal to $\sigma_{y}^{2}$ requires that the
expression in curled brackets shall be a perfect square for which the condition is

$$
\begin{aligned}
& 2\left[\frac{\gamma}{(1+\alpha)^{2}}\right]^{2}\left\{\alpha^{2}\left(1+\alpha^{2}\right) v^{6}+2 \alpha\left(1+\alpha^{2}\right) v^{5}+\left(3-\alpha^{2}-3 \alpha^{4}\right) v^{4}-4 \alpha\left(3+2 \alpha^{2}\right) v^{3}\right. \\
& \left.+\left(-2+9 \alpha^{2}+5 \alpha^{4}\right) v^{2}+2 \alpha\left(3+\alpha^{2}\right) v-\alpha^{2}\left(3+2 \alpha^{2}\right)\right\} \\
& +\frac{\gamma}{(1+\alpha)^{2}}\left\{-\alpha^{2} v^{6}-2 \alpha v^{5}+\left(-5+2 \alpha^{2}\right) v^{4}+12 \alpha v^{3}-\left(2+9 \alpha^{2}\right) v^{2}-2 \alpha v\right. \\
& \left.+3+4 a^{2}\right\}+v^{4}+2 v^{2}-1=0
\end{aligned}
$$

Now $\sigma_{y}^{2}=\frac{\sigma^{2}}{N} \cdot \frac{(1+\alpha)^{2}}{\gamma}$ is the maximum which we want to make as low as possible, hence we have for a certain $\alpha$ to find the $v$ for which $\frac{\gamma}{(1+\alpha)^{2}}$ as given by (88) is a maximum.

We shall examine the cases $\alpha=\cdot 5$ and $\alpha=\cdot 9$.

(10) For $\alpha=.5$ (88) takes the form

$$
\begin{aligned}
& {\left[\frac{\gamma}{(1+\alpha)^{2}}\right]^{2}\left\{\cdot 625 v^{6}+2 \cdot 5 v^{5}+5 \cdot 125 v^{4}-14 v^{3}+1 \cdot 125 v^{2}+6 \cdot 5 v-1 \cdot 75\right\}} \\
& \quad+\frac{\gamma}{(1+\alpha)^{2}}\left\{-\cdot 25 v^{6}-v^{5}-4 \cdot 5 v^{4}+6 v^{3}-4 \cdot 25 v^{2}-v+4\right\}+v^{4}+2 v^{2}-1=0,
\end{aligned}
$$

which differentiated with regard to $v$ gives

$$
\begin{aligned}
& {\left[\frac{\gamma}{(1+\alpha)^{2}}\right]^{2}\left\{3 \cdot 75 v^{6}+12 \cdot 5 v^{4}+20 \cdot 5 v^{3}-42 v^{2}+2 \cdot 25 v+6 \cdot 5\right\}} \\
& \quad+\frac{\gamma}{(1+\alpha)^{2}}\left\{-1 \cdot 5 v^{5}-5 v^{4}-18 v^{3}+18 v^{2}-8 \cdot 5 v-1\right\}+4 v\left(v^{2}+1\right)=0 .
\end{aligned}
$$


We find that these two equations have for $v=-\cdot 190$ the root $\frac{\gamma}{(1+\alpha)^{2}}=\cdot 2936$ in common which represents a maximum.

The maximum of the curve is hence $\frac{\sigma^{2}}{N} \cdot 3 \cdot 405$, which value occurs for $x= \pm 1$ and for $x=\cdot 064$ determined by (87).

The distribution of observations is

$$
\begin{aligned}
& .6607 N \text { at } 1, \\
& .0734 N \text { at }-1 \text {, }
\end{aligned}
$$

and

$$
.2659 \mathrm{~N} \text { at }-\cdot 190 \text {. }
$$

For comparison we shall consider what would result from taking for the $\phi(x)$ distribution three equally big groups of observations at $-1,0$ and 1 . This would for observations with the constant error $\sigma$ make the maximum of the curve equal to $\frac{\sigma^{2}}{N} \cdot 3$ and that multiplied by

$$
\begin{gathered}
1+2 \alpha \mu_{1}+\alpha^{2} \dot{\mu}_{2}=\frac{3 \cdot 5}{3} \\
\text { gives } \frac{\sigma^{2}}{\bar{N}} \cdot 3 \cdot 5 .
\end{gathered}
$$

The actual distribution $\psi(x)$ would be

$$
\begin{aligned}
& .6429 N \text { at } 1, \\
& .0714 N \text { at }-1 \text {, } \\
& .2857 N \text { at } 0 .
\end{aligned}
$$

and

This last distribution only makes the maximum $\sigma_{y}^{2}$ about 3 per cent. greater than the value which we obtained by our special distribution and it will therefore for most practical cases be as useful.

(11) When $\alpha=.9$ we find for (87),

$$
\begin{aligned}
& {\left[\frac{\gamma}{(1+\alpha)^{2}}\right]^{2}\left\{2 \cdot 9322 v^{6}+6 \cdot 516 v^{5}+\cdot 4434 v^{4}-33 \cdot 264 v^{3}+17 \cdot 141 v^{2}+13 \cdot 716 v-7 \cdot 4844\right\}} \\
& \quad+\frac{\gamma}{(1+\alpha)^{2}}\left\{-\cdot 81 v^{6}-1 \cdot 8 v^{5}-3 \cdot 38 v^{4}+10 \cdot 8 v^{3}-9 \cdot 29 v^{2}-1 \cdot 8 v+6 \cdot 24\right\} \\
& \quad+v^{4}+2 v^{2}-1=0
\end{aligned}
$$

which differentiated with regard to $v$ gives

$$
\begin{aligned}
& {\left[\frac{\gamma}{(1+\alpha)^{2}}\right]^{2}\left\{17 \cdot 5932 v^{5}+32 \cdot 58 v^{4}+1 \cdot 7736 v^{3}-99 \cdot 792 v^{2}+34 \cdot 282 v+13 \cdot 716\right\}} \\
& \quad+\frac{\gamma}{(1+\alpha)^{2}}\left\{-4 \cdot 86 v^{5}-9 v^{4}-13 \cdot 52 v^{3}+32 \cdot 4 v^{2}-18 \cdot 58 v-1 \cdot 8\right\}+4 v\left(v^{2}+1\right)=0 .
\end{aligned}
$$

For $v=-\cdot 354$ these two equations have the root $\frac{\gamma}{(1+\alpha)^{2}}=\cdot 23214$ in common which is therefore the maximum of $\frac{\gamma}{(1+\alpha)^{2}}$. 
The maximum of the corresponding $\sigma_{y}^{2}$ is hence

$$
\frac{\sigma^{2}}{N} \cdot \frac{(1+\alpha)^{2}}{\gamma}=\frac{\sigma^{2}}{N} \cdot 4 \cdot 308
$$

From (87) we find that it occurs at $x=\cdot 125$ as well as at $x= \pm 1$. The distribution of observations is then

and

$$
\begin{aligned}
& .8380 N \text { at } 1, \\
& .0023 N \text { at }-1, \\
& .1597 N \text { at }-.354 \text {. }
\end{aligned}
$$

Comparing again with a distribution consisting of three groups of observations at $-1,0$ and 1 with frequencies proportional to the squared standard deviations at these places we find that the distribution would be

and

$$
\begin{aligned}
& .7814 N \text { at } 1, \\
& .0022 N \text { at }-1, \\
& .2164 N \text { at } 0,
\end{aligned}
$$

and the maximum of $\sigma_{y}^{2}$ would be

$$
\frac{\sigma^{2}}{N} \cdot 3\left(1+2 \alpha \mu_{1}+\alpha^{2} \mu_{2}\right)=\frac{\sigma^{2}}{N} \cdot 4 \cdot 62 .
$$

We thus find that by our special distribution the maximum of $\sigma_{y}^{2}$ was 7 per cent. lower, the choice of that distribution would thus permit us to reduce the total number of observations at the same rate without raising the maximum of $\sigma_{y}^{2}$.

(12) The result of these investigations is that the maximum $\sigma_{y}$ obtained from the best three groups of observations differs so little from that obtained from three groups at $-1,0$ and 1 that the first grouping only in quite exceptional practice would be preferred.

We shall therefore in Table VI give the maximum $\sigma_{y}$ arrived at from the following three distributions: (1) three groups of observations at $-1,0$ and 1 in numbers proportional to the squared standard deviations at these places, (2) a distribution for which $\phi(x)=\frac{N}{2}$, and (3) a distribution for which $\phi(x)=\cdot 2797 \mathrm{~N}$ with additional clusters $\cdot 2203 \mathrm{~N}$ at \pm 1 (see Table II, p. 50).

Both in Table $\mathrm{V}$ and in Table VI the difference between the two first maxima as a proportion of the first decreases with increasing $a$ so that the distribution with uniform $\phi(x)$ is more profitable for $a>0$ than for observations with constant errors.

VIII. Best distribution of observations for determining a single constant of the function.

(1) Our choice of observations has hitherto aimed at giving within the working range of observations a determination of the function as accurate and uniform as possible. We shall now consider what is the best choice of observations for 
KiRstine SMith

TABLE VI.

\begin{tabular}{|c|c|c|c|c|}
\hline$\alpha$ & $\begin{array}{l}\text { Maximum of } \\
\sigma_{y} \frac{\sqrt{ } N}{\sigma} \text { from } \\
\text { three groups } \\
\text { at } 0 \text { and } \pm 1\end{array}$ & $\begin{array}{l}\text { Maximum of } \sigma_{y} \frac{\sqrt{ } N}{\sigma} \\
\text { from distribution } \\
\text { for which } \phi(x)=\frac{N}{2}\end{array}$ & $\begin{array}{l}\text { Maximum of } \sigma_{y} \frac{\sqrt{ } N}{\sigma} \\
\text { from distribution for } \\
\text { which } \phi(x)=\cdot 2797 N \\
\text { and clusters at } \pm 1\end{array}$ & $\begin{array}{l}\text { Maximum of } \\
\sigma_{y} \frac{\sqrt{ } N}{\sigma} \text { from } \\
\text { best three } \\
\text { groups }\end{array}$ \\
\hline $\begin{array}{l}.0 \\
.1 \\
.2 \\
.3 \\
.4 \\
.5 \\
.6 \\
.7 \\
.8 \\
.9\end{array}$ & $\begin{array}{l}1 \cdot 732 \\
1 \cdot 738 \\
1 \cdot 755 \\
1 \cdot 783 \\
1 \cdot 822 \\
1 \cdot 871 \\
1 \cdot 929 \\
1 \cdot 995 \\
2 \cdot 069 \\
2 \cdot 149\end{array}$ & $\begin{array}{l}3 \cdot 000 \\
3 \cdot 005 \\
3 \cdot 020 \\
3 \cdot 045 \\
3 \cdot 079 \\
3 \cdot 122 \\
3 \cdot 175 \\
3 \cdot 236 \\
3 \cdot 304 \\
3 \cdot 381\end{array}$ & $\begin{array}{l}1 \cdot 862 \\
1 \cdot 868 \\
1 \cdot 886 \\
1 \cdot 914 \\
1 \cdot 954 \\
2 \cdot 003 \\
2 \cdot 062 \\
2 \cdot 129 \\
2 \cdot 205 \\
2 \cdot 287\end{array}$ & $\begin{array}{l}\bar{Z} \\
\bar{Z} \\
\overline{1 \cdot 845} \\
\overline{-} \\
\overline{2} \cdot 076\end{array}$ \\
\hline
\end{tabular}

determining a single constant of the function. The investigations will be carried out for functions of the first and of the second degree for which the standard deviations of the observations are

or

$$
\begin{aligned}
& s_{y}=\sigma\left(1+\alpha x^{2}\right), \quad \alpha>-1 \\
& s_{y}=\sigma(1+\alpha x), \quad 1>a \geqq 0 .
\end{aligned}
$$

We have in (3) of Section I given the formula (8) for $\sigma_{\omega_{p}}^{2}$ and shall here give only the form to which it is transferred by putting

$$
\begin{aligned}
\psi(x) & =k \phi(x) f(x), \\
\frac{1}{k} & =\frac{1}{N} \int \phi(x) \cdot f(x) d x .
\end{aligned}
$$

The formula analogous to that given for $\sigma_{y}^{2}(66)$ is

$$
\begin{array}{ccccccccc}
\sigma_{a_{p}}^{2} \cdot \frac{N}{\sigma^{2}} k & 0 & 0 & 0 & \cdots \cdots & 1 & \cdots \cdots & 0 \\
0 & 1 & \mu_{1} & \mu_{2} & \cdots \cdots & \mu_{p} & \cdots \cdots & \mu_{n} \\
0 & \mu_{1} & \mu_{2} & \mu_{3} & \cdots \cdots & \mu_{p+1} & \cdots \cdots & \mu_{n+1} \\
0 & \mu_{2} & \mu_{3} & \mu_{4} & \cdots \cdots & \mu_{p+2} & \cdots \cdots & \mu_{n+2} \\
\vdots & \vdots & \vdots & \vdots & & \vdots & & \vdots \\
1 & \mu_{p} & \mu_{p+1} & \mu_{p+2} & \cdots \cdots & \mu_{2 p} & \cdots \cdots & \mu_{n+p} \\
\vdots & \vdots & \vdots & \vdots & & \vdots & & \vdots \\
0 & \mu_{n} & \mu_{n+1} & \mu_{n+2} & \cdots \cdots & \mu_{p+n} & \cdots \cdots & \mu_{2 n}
\end{array} \mid=0 \quad \ldots(89) .
$$

(2) For a function of the first degree

$$
y=a_{0}+a_{1} x
$$

for which the standard deviation of an observation is

and therefore

$$
\begin{gathered}
s_{y}=\sigma\left(1+\alpha x^{2}\right), \quad a>-1, \\
\frac{1}{k}=1+2 \alpha \mu_{2}+a^{2} \mu_{4},
\end{gathered}
$$


74 Choice in the Distribution of Observations

we find, according to (89),

and

$$
\begin{aligned}
& \sigma_{a_{0}}^{2}=\frac{\sigma^{2}}{N}\left(1+2 a \mu_{2}+\alpha^{2} \mu_{4}\right)\left(1+\frac{\mu_{1}^{2}}{\mu_{2}-\mu_{1}^{2}}\right) \ldots \ldots \ldots \ldots \ldots \ldots \ldots(90), \\
& \sigma_{a_{1}}^{2}=\frac{\sigma^{2}}{N}\left(1+2 \alpha \mu_{2}+\alpha^{2} \mu_{4}\right) \frac{1}{\mu_{2}-\mu_{1}^{2}}
\end{aligned}
$$

As for any skew distribution of observations we can find a corresponding symmetrical distribution with the same $\mu_{2}$ and $\mu_{4}$, both these expressions are a minimum for $\mu_{1}=0$.

We have already shown in (2) of Section VII that any possible values of $\mu_{2}$ and $\mu_{4}$ can be produced by three symmetrical groups of observations, so that by introducing the variables $v$ and $\gamma$ determined by

and

$$
\begin{aligned}
\mu_{2} & =v^{2} \gamma, \\
\mu_{4} & =v^{4} \gamma, \\
v^{2} & =1, \\
0 & \approx \gamma \equiv 1,
\end{aligned}
$$

and limited by

we do not leave out any possibilities.

From (90) we then get

$$
\sigma_{a_{0}}^{2}=\frac{\sigma^{2}}{N}\left(1+2 \alpha \gamma v^{2}+\alpha^{2} \gamma v^{4}\right)
$$

which for $a>0$ is a minimum when $\gamma=v^{2}=0$, and for $a=0$ is $\frac{\sigma^{2}}{N}$ for any $\gamma$ and $v^{2}$.

For $\alpha<0$ we find, since

$$
\frac{d \sigma_{a_{0}}^{2}}{d v^{2}}=\frac{\sigma^{2}}{N} 2 \alpha \gamma\left(1+\alpha v^{2}\right) \text { and } v^{2}<-\frac{1}{\alpha},
$$

that for a constant $\gamma, \sigma_{a_{0}}^{2}$ has the least value when $v^{2}$ is as great as possible, that is for $v^{2}=1$.

The minimum of $\sigma_{a_{0}}^{2}$ is then

$$
\sigma_{\iota_{0}}^{2}=\frac{\sigma^{2}}{N}\{1+(2+\alpha) \alpha \gamma\}
$$

which, since $\alpha(2+\alpha)<0$, is a minimum when $\gamma$ takes its greatest possible value 1 .

The minimum is thus

Hence we conclude that:

$$
\sigma_{a_{0}}^{2}=\frac{\sigma^{2}}{N}(1+\alpha)^{2}
$$

when $a>0, \sigma_{\iota_{0}}^{2}$ is a minimum and equal to $\frac{\sigma^{2}}{N}$ for $N$ observations at $x=0$,

when $\alpha=0, \sigma_{a_{0}}^{2}$ is a minimum and equal to $\frac{\sigma^{2}}{N}$ for any distribution for which $\mu_{1}=0$, and

when $a<0, \sigma_{\sigma_{0}}^{2}$ is a minimum and equal to $\frac{\sigma^{2}}{\bar{N}}(1+\alpha)^{2}$ for two equally big groups of observations at \pm 1 . 
(3) When we introduce $\mu_{1}=0, \mu_{2}=\gamma v^{2}$ and $\mu_{4}=\gamma v^{4}$ in (91) we get

$$
\sigma_{a_{1}}^{2}=\frac{\sigma^{2}}{N}\left(1+2 \alpha \gamma v^{2}+\alpha^{2} \gamma v^{4}\right) \frac{1}{\gamma v^{2}} .
$$

This for constant $v^{2}$ is a minimum when $\gamma=1$ and then equal to

$$
\sigma_{a_{1}}^{2}=\frac{\sigma^{2}}{N}\left(1+2 \alpha v^{2}+\alpha^{2} v^{4}\right) \frac{1}{v^{2}}
$$

As

$$
\frac{d \sigma_{a_{1}}^{2}}{d v^{2}}=\frac{\sigma^{2}}{N}\left(\alpha^{2}-\frac{1}{v^{4}}\right)
$$

$v^{2}= \pm \frac{1}{\alpha}$ when possible, that is for $\alpha \equiv 1$ determines a minimum, while for $a<1, \sigma_{a_{1}}^{2}$ reaches its lowest value for $v^{2}=1$. From (92) we find for $\alpha \equiv 1$ the minimum

and for $a<1$ the minimum

$$
\sigma_{a_{1}}^{2}=\frac{\sigma^{2}}{N} \cdot 4 \alpha
$$

$$
\sigma_{a_{1}}^{2}=\frac{\sigma^{2}}{N}(1+\alpha)^{2},
$$

both formulae giving $\sigma_{a_{1}}^{2}=\frac{\sigma^{2}}{N} \cdot 4$ for $\alpha=1$.

Our results are accordingly:

when $\alpha>1, \sigma_{\omega_{1}}^{2}$ is a minimum and equal to $\frac{\sigma^{2}}{N}$. $4 \alpha$ for two equally big groups of observations at $x= \pm \frac{1}{\alpha}$ or for any distribution with the same $\mu_{2}$ and $\mu_{4}$,

and when $\alpha \equiv 1, \sigma_{a_{1}}^{2}$ is a minimum and equal to $\frac{\sigma^{2}}{N}(1+\alpha)^{2}$ for two equally big groups of observations at $x= \pm 1$.

We see that for $\alpha \bar{\Sigma} 0$ two equally big groups of observations at \pm 1 make both $\sigma_{\iota_{0}}^{2}$ and $\sigma_{a_{1}}^{2}$ minima and these groups in addition form the distribution for which $\sigma_{\|}^{2}$ has the lowest maximum within the possible range of observations.

(4) For a function of the second degree

$$
y=a_{0}+a_{1} x+a_{2} x^{2} .
$$

with the standard deviations of observations

$$
\begin{gathered}
\qquad \begin{array}{c}
s_{y}=\sigma\left(1+\alpha x^{2}\right), \quad \alpha>-1, \\
\text { and therefore } \\
\frac{1}{k}=1+2 \alpha \mu_{2}+\alpha^{2} \mu_{4},
\end{array}
\end{gathered}
$$

we find, from (89),

$$
\begin{aligned}
\sigma_{a_{0}}^{2} & =\frac{\sigma^{2}}{N}\left(1+2 \alpha \mu_{2}+\alpha^{2} \mu_{4}\right) \cdot \frac{\mu_{2} \mu_{4}-\mu_{3}^{2}}{\mu_{2} \mu_{4}-\mu_{2}^{3}-\mu_{3}^{2}+2 \mu_{1} \mu_{2} \mu_{3}-\mu_{1}^{2} \mu_{4}} \ldots \ldots . .(93), \\
\text { and } \quad \sigma_{a_{1}}^{2} & =\frac{\sigma^{2}}{N}\left(1+2 \alpha \mu_{2}+\alpha^{2} \mu_{4}\right) \cdot \frac{\mu_{4}-\mu_{2}^{2}}{\mu_{2} \mu_{4}-\mu_{2}^{3}-\mu_{3}^{2}+2 \mu_{1} \mu_{2} \mu_{3}-\mu_{1}^{2} \mu_{4}} \ldots \ldots \ldots(94), \\
\sigma_{a_{2}}^{2} & =\frac{\sigma^{2}}{N}\left(1+2 \alpha \mu_{2}+\alpha^{2} \mu_{4}\right) \cdot \frac{\mu_{2}-\mu_{1}^{2}}{\mu_{2} \mu_{4}-\mu_{2}^{3}-\mu_{3}^{2}+2 \mu_{1} \mu_{2} \mu_{3}-\mu_{1}^{2} \mu_{4}} \ldots \ldots \ldots(9 \check{5}) .
\end{aligned}
$$


We shall prove that the last factor of each of these formulae is a minimum for

$$
\mu_{1}=\mu_{3}=0 \text {. }
$$

To prove this for (93) we consider the difference

$$
\frac{\mu_{2} \mu_{4}}{\mu_{2} \mu_{4}-\mu_{2}^{3}}-\frac{\mu_{3}^{2}}{\mu_{3}^{2}-2 \mu_{1} \mu_{2} \mu_{3}+\mu_{1}^{2} \mu_{4}}=\frac{\mu_{2}\left(\mu_{2} \mu_{3}-\mu_{1} \mu_{4}\right)^{2}}{\mu_{2}\left(\mu_{4}-\mu_{2}^{2}\right)\left[\left(\mu_{3}-\mu_{1} \mu_{2}\right)^{2}+\mu_{1}^{2}\left(\mu_{4}-\mu_{2}^{2}\right)\right]}>0,
$$

from which follows

$$
\frac{\mu_{2} \mu_{4}-\mu_{3}^{2}}{\mu_{2} \mu_{4}-\mu_{2}^{3}-\mu_{3}^{2}+2 \mu_{1} \mu_{2} \mu_{3}-\mu_{1}^{2} \mu_{4}}>\frac{\mu_{2} \mu_{4}}{\mu_{2} \mu_{4}-\mu_{2}^{3}}>\frac{\mu_{3}^{2}}{\mu_{3}^{2}-2 \mu_{1} \mu_{2} \mu_{3}+\mu_{1}^{2} \mu_{4}} .
$$

For (94) it is at once clear that

$$
\frac{\mu_{4}-\mu_{2}^{2}}{\mu_{2} \mu_{4}-\mu_{2}^{3}-\left[\left(\mu_{3}-\mu_{1} \mu_{2}\right)^{2}+\mu_{1}^{2}\left(\mu_{4}-\mu_{2}^{2}\right)\right]}>\frac{\mu_{4}-\mu_{2}^{2}}{\mu_{2} \mu_{4}-\mu_{2}^{3}} .
$$

For the case of (95) we compare

$$
\frac{\mu_{2}}{\mu_{2} \mu_{4}-\mu_{2}^{3}} \text { and } \frac{\mu_{1}^{2}}{\mu_{3}^{2}-2 \mu_{1} \mu_{2} \mu_{3}+\mu_{1}^{2} \mu_{4}},
$$

and we find the difference

and hence

$$
\frac{1}{\mu_{4}-\mu_{2}^{2}}-\frac{1}{\mu_{4}-\mu_{2}^{2}+\left(\frac{\mu_{3}}{\mu_{1}}-\mu_{2}\right)^{2}}>0
$$

$$
\frac{\mu_{2}-\mu_{1}^{2}}{\mu_{2} \mu_{4}-\mu_{2}^{3}-\mu_{3}^{2}+2 \mu_{1} \mu_{2} \mu_{3}-\mu_{1}^{2} \mu_{4}}>\frac{\mu_{2}}{\mu_{2} \mu_{4}-\mu_{2}^{3}}>\frac{\mu_{1}^{2}}{\mu_{3}^{2}-2 \mu_{1} \mu_{2} \mu_{3}+\mu_{1}^{2} \mu_{4}} .
$$

It is thus proved for the three formulae that a distribution of observations for which $\mu_{1}=\mu_{3}=0$ gives lower values than any distribution with the same $\mu_{2}$ and $\mu_{4}$ as the former and with $\mu_{1} \leqq 0, \mu_{3} \gtrless 0$.

Hence our problem is reduced to finding the $\mu_{2}$ and $\mu_{4}$ which make the following expressions minima :

$$
\begin{aligned}
\sigma_{\iota_{0}}^{2} & =\frac{\sigma^{2}}{N}\left(1+2 \alpha \mu_{2}+\alpha^{2} \mu_{4}\right) \frac{\mu_{4}}{\mu_{4}-\mu_{2}^{2}} \\
\sigma_{a_{1}}^{2} & =\frac{\sigma^{2}}{N}\left(1+2 \alpha \mu_{2}+\alpha^{2} \mu_{4}\right) \frac{1}{\mu_{2}} \ldots . \\
\sigma_{a_{2}}^{2} & =\frac{\sigma^{2}}{N}\left(1+2 \alpha \mu_{2}+\alpha^{2} \mu_{4}\right) \frac{1}{\mu_{4}-\mu_{2}^{2}}
\end{aligned}
$$

(5) Introducing $\mu_{2}=\gamma v^{2}$ and $\mu_{4}=\gamma v^{4}$ in (96) we get

$$
\sigma_{a_{0}}^{2}=\frac{\sigma^{2}}{N}\left(1+\frac{\gamma}{1-\gamma}\left(1+\alpha v^{2}\right)^{2}\right)
$$

which is seen to be $>\frac{\sigma^{2}}{N}$ except when $\gamma=0$.

Hence the minimum value of $\sigma_{a_{0}}^{2}=\frac{\sigma^{2}}{\bar{N}}$ can only be obtained by taking all the observations at $x=0$.

(97) is identical with (91) for $\mu_{1}=0$. The conditions for a minimum of $\sigma_{a_{1}}^{2}$ are 


\section{Kirstine Sмith}

therefore the same for a function of the second degree as for a function of the first degree. That is, when $a>1, \sigma_{\omega_{1}}^{2}$ is a minimum and equal to $\frac{\sigma^{2}}{N} \cdot 4 a$ for two equally big groups of observations at $x= \pm \frac{1}{\alpha}$, or for any distribution with the same $\mu_{2}$ and $\mu_{4}$, and when $a \bar{\gtrless} 1, \sigma_{a_{1}}^{2}$ is a minimum and equal to $\frac{\sigma^{2}}{N}(1+\alpha)^{2}$ for two equally big groups of observations at $x= \pm 1$.

With the variates $\gamma$ and $v(98)$ takes the form

$$
\sigma_{a_{2}}^{2}=\frac{\sigma^{2}}{N}\left(1+2 \alpha \gamma v^{2}+\alpha^{2} \gamma v^{4}\right) \frac{1}{v^{4} \gamma(1-\gamma)} .
$$

By differentiating with regard to $v^{2}$ we get

$$
\frac{d \sigma_{a_{2}}^{2}}{d v^{2}}=\frac{\sigma^{2}}{N} \cdot \frac{2}{\gamma(1-\gamma) v^{6}}\left(-1-a \gamma v^{2}\right)
$$

which is negative for any $\alpha, v$ and $\gamma$ within our limits.

For constant $\gamma, \sigma_{a_{2}}^{2}$ is therefore least when $v^{2}=1$ and the minimum value is

$$
\sigma_{a_{2}}^{2}=\frac{\sigma^{2}}{N}\left(\frac{1}{\gamma}+2 \alpha+\alpha^{2}\right) \frac{1}{1-\gamma}
$$

This is again a minimum when

$$
\frac{d \sigma_{a_{2}}^{2}}{d \gamma}=\frac{\sigma^{2}}{N} \cdot \frac{1}{\gamma^{2}(1-\gamma)^{2}}\left\{\alpha(2+\alpha) \gamma^{2}+2 \gamma-1\right\}=0,
$$

that is for $\gamma=\frac{1}{2+\alpha}$ which gives a minimum both for positive and negative $\alpha$.

Thus the distribution that makes $\sigma_{a_{2}}^{2}$ a minimum has a $\phi(x)$-distribution consisting of $\frac{N}{2(2+\alpha)}$ observations at -1 and 1 and $\frac{1+\alpha}{2+\alpha} N$ observations at 0 .

We have

and

The relation

then gives us

and

$$
\mu_{2}=\mu_{4}=\frac{1}{2+\alpha}
$$$$
\frac{1}{k}=(1+\alpha) \text {. }
$$

$$
\psi(x)=k \phi(x) f(x)
$$$$
\psi(0)=\frac{N}{2+\alpha}
$$

$$
\psi( \pm 1)=\frac{1+\alpha}{2(2+\alpha)} . N
$$

From (99) we find the minimum value

$$
\sigma_{a_{2}}^{2}=\frac{\sigma^{2}}{N}(2+\alpha)^{2} .
$$

Our result is thus that $\sigma_{a_{2}}^{2}$ is a minimum and equal to $\frac{\sigma^{2}}{N}(2+\alpha)^{2}$ for a distribution consisting of $\frac{N}{2+\alpha}$ observations at 0 and $\frac{1+\alpha}{2(2+\alpha)} N$ at \pm 1 . 
(6) When the standard deviation of an observation is

we have

$$
\begin{gathered}
s_{y}=\sigma(1+\alpha x) \text { and } 0 \leqq \alpha<1, \\
\frac{1}{k}=1+2 \alpha \mu_{1}+\alpha^{2} \mu_{2},
\end{gathered}
$$

and according to (89) we find for a function of the first degree

and

$$
\begin{aligned}
& \sigma_{a_{0}}^{2}=\frac{\sigma^{2}}{N}\left(1+2 \alpha \mu_{1}+\alpha^{2} \mu_{2}\right) \frac{\mu_{2}}{\mu_{2}-\mu_{1}^{2}} \\
& \sigma_{a_{1}}^{2}=\frac{\sigma^{2}}{N}\left(1+2 \alpha \mu_{1}+\alpha^{2} \mu_{2}\right) \frac{1}{\mu_{2}-\mu_{1}^{2}}
\end{aligned}
$$

By differentiating (100) we find

and

$$
\begin{aligned}
& \frac{d \sigma_{\sigma_{0}}^{2}}{d \mu_{1}}=\frac{\sigma^{2}}{N} \frac{2 \mu_{2}\left(1+\alpha \mu_{1}\right)\left(\mu_{1}+\alpha \mu_{2}\right)}{\left(\mu_{2}-\mu_{1}^{2}\right)^{2}} \\
& \frac{d \sigma_{\sigma_{0}}^{2}}{d \mu_{2}}=\frac{\sigma^{2}}{N} \frac{\left(\alpha \mu_{2}-2 \alpha \mu_{1}^{2}-\mu_{1}\right)\left(\mu_{1}+\alpha \mu_{2}\right)}{\left(\mu_{2}-\mu_{1}^{2}\right)^{2}} .
\end{aligned}
$$

Both of these can only be zero when

$$
\mu_{1}+\alpha \mu_{2}=0
$$

which is seen to determine a minimum of $\sigma_{a_{0}}^{2}$ the value of which is $\frac{\sigma^{2}}{\bar{N}}$. The condition $\mu_{2}=-\frac{\mu_{1}}{\alpha}$ can be fulfilled by an infinity of different distributions. From

$$
\begin{array}{ll} 
& 0 \bar{\Sigma} \mu_{2} \bar{\Sigma} 1 \\
\text { follows the condition } & 0 \bar{\Sigma} \mu_{1} \bar{\Sigma}-\alpha .
\end{array}
$$

We shall confine our attention to those distributions which consist of two groups of observations. Let there be $N_{\gamma}$ observations at $v_{1}$ and $(1-\gamma) N$ at $v_{2}$, we then have

$$
\begin{aligned}
& \mu_{1}=v_{z}+\gamma\left(v_{1}-v_{2}\right), \\
& \mu_{2}=v_{2}^{2}+\gamma\left(v_{1}^{2}-v_{2}^{2}\right),
\end{aligned}
$$

from which by means of (102) is found

and

$$
\begin{aligned}
\frac{\gamma}{-v_{2}\left(1+\alpha v_{2}\right)} & =\frac{1-\gamma}{v_{1}\left(1+\alpha v_{1}\right)}=\frac{1}{\left(v_{1}-v_{2}\right)\left\{1+\alpha\left(v_{1}+v_{2}\right)\right\}} \\
\frac{1}{k} & =1+\alpha \mu_{1}=\frac{\left(1+\alpha v_{1}\right)\left(1+\alpha v_{2}\right)}{1+\alpha\left(v_{1}+v_{2}\right)}
\end{aligned}
$$

Thus we find that the $\phi(x)$-distribution consists of

and

$$
\begin{aligned}
& \frac{-v_{2}\left(1+\alpha v_{2}\right)}{\left(v_{1}-v_{2}\right)\left\{1+\alpha\left(v_{1}+v_{2}\right)\right\}} N \text { at } v_{1} \\
& \frac{v_{1}\left(1+\alpha v_{1}\right)}{\left(v_{1}-v_{2}\right)\left\{1+\alpha\left(v_{1}+v_{2}\right)\right\}} N \text { at } v_{2} \text {, }
\end{aligned}
$$

while the actual distribution

$$
\psi(x)=\frac{1+\alpha\left(v_{1}+v_{2}\right)}{\left(1+\alpha v_{1}\right)\left(1+\alpha v_{2}\right)}(1+\alpha x)^{2} \phi(x)
$$




\section{KiRstine SMith}

consists of

$$
\left.\begin{array}{c}
\frac{-v_{2}\left(1+\alpha v_{1}\right)}{v_{1}-v_{2}} N \text { at } v_{1} \\
\frac{v_{1}\left(1+\alpha v_{2}\right)}{v_{1}-v_{2}} N \text { at } v_{2}
\end{array}\right\}
$$

and

We thus see that for any two points $v_{1}$ and $v_{2}$ of which one is negative and the other positive we can choose the numbers of observations so as to make $\sigma_{a_{0}}^{2}=\frac{\sigma^{2}}{\bar{N}}$ as it of course would be by taking a single group of observations at $x=0$.

(7) By differentiating (101) we get

$$
\begin{aligned}
& \frac{d \sigma_{a_{1}}^{2}}{d \mu_{1}}=\frac{\sigma^{2}}{N} \frac{2}{\left(\mu_{2}-\mu_{1}^{2}\right)^{2}}\left(1+\alpha \mu_{1}\right)\left(\mu_{1}+\alpha \mu_{2}\right) \\
& \text { and } \quad \frac{d \sigma_{a_{1}}^{2}}{d \mu_{2}}=-\frac{\sigma^{2}}{N} \frac{\left(1+\alpha \mu_{1}\right)^{2}}{\left(\mu_{2}-\mu_{1}\right)^{2}} \text {. }
\end{aligned}
$$

As the latter is always negative $\sigma_{a_{1}}^{2}$ is for constant $\mu_{1}$ least when $\mu_{2}$ has its greatest value, that is 1 .

Introducing this in (104) we get as condition for a minimum,

$$
\mu_{1}+\alpha=0 .
$$

There is only one distribution for which $\mu_{2}=1$ and $\mu_{1}=-\alpha$, and it is that consisting of two groups of observations at -1 and 1 included in the distributions examined in (6).

From (103) we find that the actual distribution consists of $\frac{1-a}{2} N$ observations at -1 and $\frac{1+\alpha}{2} N$ at 1 . The minimum of $\sigma_{a_{1}}^{2}$ is from (101) found to be $\frac{\sigma^{2}}{N}$.

The minimum $\frac{\sigma^{2}}{\bar{N}}$ of $\sigma_{a_{1}}^{2}$ can thus only be obtained by taking two groups of observations at the limits of the range with numbers proportional to the standard deviation of observations at these places. This distribution makes also $\sigma_{a_{0}}^{2}$ a minimum, but it is not, except when $\alpha=0$, the distribution which gives $\sigma_{y}^{2}$ the lowest maximum value within the possible range of observations.

(8) For a function of the second degree,

with the standard deviation

$$
y=a_{0}+a_{1} x+a_{2} x^{2}
$$

where

$$
\sigma_{y}=\sigma(1+a x),
$$

we have

$$
0 \leqq \alpha<1
$$

and from (89),

$$
\frac{1}{k}=1+2 \alpha \mu_{1}+\alpha^{2} \mu_{2} \text {, }
$$

$$
\begin{aligned}
& \sigma_{a_{0}}^{2}=\frac{\sigma^{2}}{N}\left(1+2 \alpha \mu_{1}+\alpha^{2} \mu_{2}\right) \frac{\mu_{2} \mu_{4}-\mu_{3}^{2}}{\mu_{2} \mu_{4}-\mu_{2}^{3}-\mu_{3}^{2}+2 \mu_{1} \mu_{2} \mu_{3}-\mu_{1}^{2} \mu_{4}} \ldots(105), \\
& \sigma_{a_{1}}^{2}=\frac{\sigma^{2}}{N}\left(1+2 \alpha \mu_{1}+\alpha^{2} \mu_{2}\right) \frac{\mu_{4}-\mu_{2}^{2}}{\mu_{2} \mu_{4}-\mu_{2}^{3}-\mu_{3}^{2}+2 \mu_{1} \mu_{2} \mu_{3}-\mu_{1}^{2} \mu_{4}} \ldots(106), \\
& \sigma_{a_{2}}^{2}=\frac{\sigma^{2}}{N}\left(1+2 \alpha \mu_{1}+\alpha^{2} \mu_{2}\right) \frac{\mu_{2}-\mu_{1}^{2}}{\mu_{2} \mu_{4}-\mu_{2}^{3}-\mu_{3}^{2}+2 \mu_{1} \mu_{2} \mu_{3}-\mu_{1}^{2} \mu_{4}} \ldots(107) .
\end{aligned}
$$




\section{Choice in the Distribution of Observations}

(105) may be brought into the form

$$
\sigma_{a_{0}}^{2}=\frac{\sigma^{2}}{N}\left\{1+\frac{\mu_{2} \cdot\left(\mu_{2} \mu_{4}-\mu_{3}^{2}\right)\left(\alpha+\frac{\mu_{1}}{\mu_{2}}\right)^{2}+\frac{1}{\mu_{2}}\left(\mu_{2}^{2}-\mu_{1} \mu_{3}\right)^{2}}{\mu_{2} \mu_{4}-\mu_{2}^{3}-\mu_{3}^{2}+2 \mu_{1} \mu_{2} \mu_{3}-\mu_{1}^{2} \mu_{4}}\right\},
$$

where the denominator and $\mu_{2} \mu_{4}-\mu_{3}^{2}$ are always positive. Hence the condition for $\sigma_{a_{0}}^{2}$, taking its minimum value $\frac{\sigma^{2}}{N}$, is

or

$$
\begin{aligned}
& \mu_{1}+\alpha \mu_{2}=0 \text { and } \mu_{2}^{2}-\cdots \mu_{1} \mu_{3}=0 \\
& \frac{\mu_{3}}{\mu_{2}}=\frac{\mu_{2}}{\mu_{1}}=-\frac{1}{\alpha}
\end{aligned}
$$

We shall examine the possible distributions consisting of three groups of observations with the frequencies $\gamma_{1}, \gamma_{2}$ and $\gamma_{3}$ at $v_{1}, v_{2}$ and $v_{3}$. The conditions (108) require

$$
\begin{gathered}
\frac{\gamma_{1} v_{1}^{2}+\gamma_{2} v_{2}^{2}+\gamma_{3} v_{3}^{2}}{\gamma_{1} v_{1}+\gamma_{2} v_{2}+\gamma_{3} v_{3}}=\frac{\gamma_{1} v_{1}^{3}+\gamma_{2} v_{2}^{3}+\gamma_{3} v_{3}^{3}}{\gamma_{1} v_{1}^{2}+\gamma_{2} v_{2}^{2}+\gamma_{3} v_{3}^{2}}=\frac{\gamma_{2} v_{2}^{2}\left(v_{2}-v_{1}\right)+\gamma_{3} v_{3}^{2}\left(v_{3}-v_{1}\right)}{\gamma_{2} v_{2}\left(v_{2}-v_{1}\right)+\gamma_{3} v_{3}\left(v_{3}-v_{1}\right)}=-\frac{1}{a} \\
\frac{\gamma_{1} v_{1}\left(1+a v_{1}\right)}{v_{2}-v_{3}}=\frac{\gamma_{2} v_{2}\left(1+\alpha v_{2}\right)}{v_{3}-v_{1}}=\frac{\gamma_{3} v_{3}\left(1+a v_{3}\right)}{v_{1}-v_{2}} \ldots \ldots \ldots \ldots . .(109)
\end{gathered}
$$

or

Now $\frac{v_{1}}{v_{2}-v_{3}}, \frac{v_{2}}{v_{3}-v_{1}}$ and $\frac{v_{3}}{v_{1}-v_{2}}$ can never all have the same sign and $(1+\alpha v)$ is for any $v \geqq-1$ positive, from which it follows that (109) leads to negative frequencies. Nor can (109) be satisfied by two groups of observations as $\gamma_{2}=0$ requires $v_{1}=v_{3}=0$, that is one group of observations at $x=0$ which of course gives $\sigma_{a_{0}}^{2}=\frac{\sigma^{2}}{N}$.

(9) We may write (106)

$$
\sigma_{a_{1}}^{2}=\frac{\sigma^{2}}{N}\left(\frac{1}{\mu_{2}}+\frac{1}{\mu_{2}} \cdot \frac{\left(\mu_{4}-\mu_{2}^{2}\right)\left(\mu_{1}+\alpha \mu_{2}\right)^{2}+\left(\mu_{3}-\mu_{1} \mu_{2}\right)^{2}}{\left(\mu_{4}-\mu_{2}^{2}\right)\left(\mu_{2}-\mu_{1}^{2}\right)-\left(\mu_{3}-\mu_{1} \mu_{2}\right)^{2}}\right),
$$

where the last ratio is seen to be positive unless

$$
\mu_{1}+\alpha \mu_{2}=0 \text { and } \mu_{3}-\mu_{1} \mu_{2}=0
$$

If therefore any distribution of observations can give $\frac{1}{\mu_{2}}$ its minimum value 1 and at the same time fulfil those conditions it will make $\sigma_{a_{1}}^{2}$ a minimum and equal to $\frac{\sigma^{2}}{N}$. But $\mu_{2}=1$ together with (110) lead to $\mu_{3}=\mu_{1}=-\alpha$
which require $\frac{1+\alpha}{2} N$ observations at -1 and $\frac{1-\alpha}{2} N$ at 1 , whereas the actual distribution must consist of $\frac{1-\alpha}{2} N$ observations at -1 and $\frac{1+\alpha}{2} N$ at 1 .

Thus the only distribution which makes $\sigma_{a_{1}}^{2}$ a minimum and equal to $\frac{\sigma^{2}}{N}$ is that consisting of $\frac{1-\alpha}{2} N$ observations at -1 and $\frac{1+\alpha}{2} N$ at 1 . 
Kinstine Smith

(10) The general minimum conditions for $\sigma_{a_{2}}$ cannot be found without more elaborate investigations into the possible variations of the moment coefficients than are at present available and we shall limit our research to the case of three groups of observations.

Let us suppose $\gamma_{1} N, \gamma_{2} N$ and $\left(1-\gamma_{1}-\gamma_{2}\right) N$ observations taken at $x_{1}, x_{2}$ and $x_{3}$, and let the corresponding means be $\bar{y}_{1}, \bar{y}_{2}$ and $\bar{y}_{3}$.

We then find, when

$$
\begin{gathered}
\Delta=\left(x_{1}-x_{2}\right)\left(x_{2}-x_{3}\right)\left(x_{3}-x_{1}\right), \\
a_{2}=\frac{1}{\Delta}\left\{\bar{y}_{1}\left(x_{3}-x_{2}\right)+\bar{y}_{2}\left(x_{1}-x_{3}\right)+\bar{y}_{3}\left(x_{2}-x_{1}\right)\right\},
\end{gathered}
$$

and

$$
\sigma_{a_{2}}^{2}=\frac{\sigma^{2}}{\Delta^{2} \cdot N}\left\{\frac{\left(x_{3}-x_{2}\right)^{2}\left(1+\alpha x_{1}\right)^{2}}{\gamma_{1}}+\frac{\left(x_{1}-x_{3}\right)^{2}\left(1+\alpha x_{2}\right)^{2}}{\gamma_{2}}+\frac{\left(x_{2}-x_{1}\right)^{2}\left(1+\alpha x_{3}\right)^{2}}{1-\gamma_{1}-\gamma_{2}}\right\}
$$

Differentiations first with regard to $\gamma_{1}$ and then with regard to $\gamma_{2}$ give the minimum conditions

$$
\frac{\gamma_{1}^{2}}{\left(x_{3}-x_{2}\right)^{2}\left(1+\alpha x_{1}\right)^{2}}=\frac{\gamma_{2}^{2}}{\left(x_{1}-x_{3}\right)^{2}\left(1+\alpha x_{2}\right)^{2}}=\frac{\left(1-\gamma_{1}-\gamma_{2}\right)^{2}}{\left(x_{2}-x_{1}\right)^{2}\left(1+\alpha x_{3}\right)^{2}},
$$

or, when we suppose $x_{1}<x_{2}<x_{3}$,

$$
\frac{\gamma_{1}}{\left(x_{3}-x_{2}\right)\left(1+\alpha x_{1}\right)}=\frac{-\gamma_{2}}{\left(x_{1}-x_{3}\right)\left(1+\alpha x_{2}\right)}=\frac{1-\gamma_{1}-\gamma_{2}}{\left(x_{2}-x_{1}\right)\left(1+\alpha x_{3}\right)}=\frac{1}{2\left(x_{3}-x_{1}\right)\left(1+\alpha x_{2}\right)}
$$

With these values for $\gamma_{1}$ and $\gamma_{2}$ we get from (111)

$$
\sigma_{a_{2}}^{2}=\frac{\sigma^{2}}{\bar{N}}\left\{\frac{2\left(x_{3}-x_{1}\right)\left(1+a x_{2}\right)}{\Delta}\right\}^{2}=\frac{\sigma^{2}}{N}\left\{\frac{2\left(1+a x_{2}\right)}{\left(x_{2}-x_{1}\right)\left(x_{3}-x_{2}\right)}\right\}^{2} .
$$

This for constant $x_{2}$ is obviously a minimum for $x_{1}=-1$ and $x_{3}=1$ and is then equal to

From this we find

$$
\sigma_{a_{2}}^{2}=\frac{\sigma^{2}}{N}\left\{\frac{2\left(1+a x_{2}\right)}{1-x_{2}^{2}}\right\}^{2} .
$$

which shows that

$$
\frac{d \sigma_{a_{2}}}{d x_{2}}=\frac{\sigma}{\sqrt{N}} \frac{2\left(\alpha x_{2}^{2}+2 x_{2}+\alpha\right)}{\left(1-x_{2}^{2}\right)^{2}},
$$

determines a minimum.

$$
x_{2}=\sqrt{\frac{1}{\alpha^{2}}-1}-\frac{1}{\alpha}
$$

The minimum value is

$$
\sigma_{a_{2}}^{2}=\frac{\sigma^{2}}{N}\left(1+\sqrt{1-a^{2}}\right)^{2},
$$

and the frequencies found from (112) are

$$
\frac{1}{4 \alpha} \sqrt{1-\alpha}(\sqrt{1+\alpha}-\sqrt{1-\alpha}) \cdot N \text { at }-1,
$$

Biometrika XII 


$$
\frac{1}{4 \alpha} \sqrt{ } 1+\alpha(\sqrt{1+\alpha}-\sqrt{1-\alpha}) \cdot N \text { at } 1
$$

and

$$
\frac{1}{2} N \text { at }-\frac{1}{\alpha}\left(1-\sqrt{1-\alpha^{2}}\right) \text {. }
$$

\section{Adjustment with regard to both of two variates connected by a linear relation.}

(1) The case often occurs when both of the variates observed have errors of observations of the same order so that adjustment only of one of them is unsatisfactory. We shall therefore in this section consider adjustment with regard to both of the variates and give the adjusted relation between them and the standard deviations of the constants.

Let $x^{\prime}$ be observed with the standard deviation $\sqrt{\alpha} \sigma$ and $y^{\prime}$ with the standard deviation $\sqrt{\gamma} \sigma$, we shall then for the sake of greater perspicuity exchange the variates for $x=\frac{x^{\prime}}{\sqrt{ } \alpha}$ and $y=\frac{y^{\prime}}{\sqrt{ } \gamma}$ so that both of our variates have the same standard deviation $\sigma$. Let $\frac{1}{N} \Sigma\left\{x^{r} y^{s}\right\}$ taken over the $N$ pairs of observations be denoted by $\mu_{r, s}$, we then find, by adjusting only the $y$ 's according to (3),

or

$$
\begin{aligned}
& \left|\begin{array}{ccc}
y & 1 & x \\
\mu_{01} & 1 & \mu_{10} \\
\mu_{11} & \mu_{10} & \mu_{20}
\end{array}\right|=0, \\
& y-\mu_{01}=\frac{\mu_{11}-\mu_{01} \mu_{10}}{\mu_{20}-\mu_{10}^{2}}\left(x-\mu_{10}\right)
\end{aligned}
$$

By adjusting only the $x$ 's we get

$$
y-\mu_{01}=\frac{\mu_{02}-\mu_{01}^{2}}{\mu_{11}-\mu_{01} \mu_{10}}\left(x-\mu_{10}\right)
$$

which only coincide with (113) when

$$
\left(\mu_{20}-\mu_{10}^{2}\right)\left(\mu_{02}-\mu_{01}^{2}\right)=\left(\mu_{11}-\mu_{01} \mu_{10}\right)^{2} \text {, }
$$

that is when there is perfect correlation between $x$ and $y$ and no casual errors of observation.

(2) Adjusting at the same time with regard to $x$ and $y$ may be transformed to the problem of finding the straight line for which the sum of the squared distances of the observed points $(x, y)$ is a minimum.

Let the line sought be

$$
x \cos v+y \sin v+p=0 .
$$

The sum which we want to make a minimum is then

$$
\begin{gathered}
S=\mu_{20} \cos ^{2} v+\mu_{02} \sin ^{2} v+2 \mu_{11} \cos v \sin v+2 p \mu_{10} \cos v+2 p \mu_{01} \sin v+p^{2}, \\
\frac{d S}{d p}=0 \text { requires } \quad p=-\mu_{10} \cos v-\mu_{01} \sin v,
\end{gathered}
$$

indicating that the line passes through the mean $\left(\mu_{10}, \mu_{01}\right)$; this determines a minimum for constant $v$. 


\section{KIRSTINE SHITH}

The corresponding $S$ is

$$
S=\left(\mu_{20}-\mu_{10}^{2}\right) \cos ^{2} v+\left(\mu_{02}-\mu_{01}^{2}\right) \sin ^{2} v+2\left(\mu_{11}-\mu_{01} \mu_{10}\right) \cos v \sin v \ldots \ldots(115),
$$

which differentiated with regard to $v$ gives

$$
\frac{d S}{d v}=-\left\{\mu_{20}-\mu_{10}^{2}-\left(\mu_{02}-\mu_{01}^{2}\right)\right\} \sin 2 v+2\left(\mu_{11}-\mu_{01} \mu_{10}\right) \cos 2 v .
$$

It thus follows that

or

$$
\tan 2 v=\frac{2\left(\mu_{11}-\mu_{01} \mu_{10}\right)}{\mu_{20}-\mu_{10}^{2}-\left(\mu_{02}-\mu_{01}^{2}\right)}=\frac{2 \tan v}{1-\tan ^{2} v},
$$

$$
\tan v=\frac{\frac{1}{2}\left\{\mu_{02}-\mu_{01}^{2}-\left(\mu_{20}-\mu_{10}^{2}\right) \pm \sqrt{\left[\mu_{02}-\mu_{01}^{2}-\left(\mu_{20}-\mu_{10}^{2}\right)\right]^{2}+4\left[\mu_{11}-\mu_{10} \mu_{01}\right]^{2}}\right\}}{\mu_{11}-\mu_{01} \mu_{10}}
$$

determine a maximum and a minimum of $S$.

Substituting in (115) we find

$$
S=\frac{1}{2}\left\{\mu_{20}-\mu_{10}^{2}+\mu_{02}-\mu_{01}^{2} \pm \sqrt{\left.\left[\mu_{20}-\mu_{10}^{2}-\left(\mu_{02}-\mu_{01}^{2}\right)\right]^{2}+4\left[\mu_{11}-\mu_{01} \mu_{10}\right]^{2}\right\}},\right.
$$
so that the minimum corresponds to the negative sign of the root in (116).

The adjusted function connecting $x$ and $y$ is hence a line through the general mean forming an angle $u$ with the $x$-axis which is determined by

$\tan u=-\cot v=\frac{\mu_{02}-\mu_{01}^{2}-\left(\mu_{20}-\mu_{10}^{2}\right)+\sqrt{\left[\mu_{20}-\mu_{10}^{2}-\left(\mu_{02}-\mu_{01}^{2}\right)\right]^{2}+4\left[\mu_{11}-\mu_{01} \mu_{10}\right]^{2}}}{2\left(\mu_{11}-\mu_{01} \mu_{10}\right)}$

For the variates $x^{\prime}$ and $y^{\prime}$ there must to this value of the tangent be added the factor $\sqrt{ } \frac{\gamma}{\alpha}$, expressed by the moment coefficients of $x^{\prime}$ and $y^{\prime}$ we therefore find $\tan u=\frac{\alpha\left(\mu_{02}^{\prime}-\mu_{01}^{2}\right)-\gamma\left(\mu_{20}^{\prime}-\mu_{10}^{\prime 2}\right)+\sqrt{\left[\gamma\left(\mu_{20}^{\prime}-\mu_{10}^{\prime 2}\right)-\alpha\left(\mu_{02}^{\prime}-\mu_{01}^{\prime 2}\right)\right]^{2}+4 \alpha \gamma\left[\mu_{11}^{\prime}-\mu_{01}^{\prime} \mu_{10}^{\prime}\right]^{2}}}{2 \alpha\left(\mu_{11}^{\prime}-\mu_{01}^{\prime} \mu_{10}^{\prime}\right)}$

(3) We shall prove that the line is situated between the two regression curves (113) and (114).

Making $\left(\mu_{10}, \mu_{01}\right)$ the zero point of the coordinates, the three tangents to be compared are

$$
\frac{\mu_{11}}{\mu_{20}}, \frac{\mu_{02}}{\mu_{11}} \text { and } \frac{1}{2 \mu_{11}}\left\{\mu_{02}-\mu_{20}+\sqrt{ }\left(\mu_{02}-\mu_{20}\right)^{2}+4 \mu_{11}^{2}\right\}=\tan u
$$

where the $\mu$ 's now are the moment coefficients about the mean.

According to $\mu_{11} \gtrless 0$ we have

since

$$
\frac{\mu_{11}}{\mu_{20}}>\frac{\mu_{02}}{\mu_{11}}
$$

As

$$
\mu_{11}^{2}<\mu_{20} \cdot \mu_{02} \text {. }
$$

we have

$$
\sqrt{\left(\mu_{02}-\mu_{20}\right)^{2}+4 \mu_{11}^{2}}<\mu_{02}+\mu_{20} \text {, }
$$

$$
\tan u \lesseqgtr \frac{\mu_{02}}{\mu_{11}} .
$$


It rests to compare $\tan u$ and $\frac{\mu_{11}}{\mu_{20}}$, we find

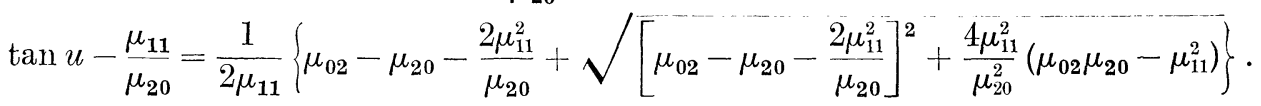

The factor in curled brackets is hence positive and we have $\tan u>$ or $<\frac{\mu_{11}}{\mu_{\mathbf{2 0}}}$ according as

$$
\mu_{11}>\text { or }<0 \text {, }
$$

we have thus proved that

$$
\frac{\mu_{11}}{\mu_{20}} \lesseqgtr \tan u \lesseqgtr \frac{\mu_{02}}{\mu_{11}} .
$$

(4) In order to find the standard deviations of the constants of the line we shall express the observations, the standard deviations of which are $\sqrt{\alpha} \sigma$ and $\sqrt{\gamma} \sigma$, by a parameter $r$ to get an equation for each observation.

Suppose

$$
\begin{aligned}
& x_{i}=a+r_{i} \cos u, \\
& y_{i}=b+r_{i} \sin u,
\end{aligned}
$$

and suppose we have a good approximation for $a, b, u, r_{1}, r_{2} \ldots \ldots r_{N}$ from which is calculated $x$ and $y$ corresponding to the observations. The differences between observed and calculated $x$ and $y$ can then be expressed by

$$
\left.\begin{array}{l}
\Delta x_{i}=\Delta a-r_{i} \sin u \cdot \Delta u+\cos u \cdot \Delta r_{i} \\
\Delta y_{i}=\Delta b+r_{i} \cos u . \Delta u+\sin u \cdot \Delta r_{i}
\end{array}\right\}
$$

and we can carry out an adjustment, $\Delta a, \Delta b, \Delta u, \Delta r_{1}, \Delta r_{2} \ldots \Delta r_{N}$ being the elements.

The normal equations are:

$$
\begin{aligned}
& \frac{1}{a} \Sigma\left\{x_{i}\right\}=\frac{N}{a} \Delta a+0 . \Delta b-\Sigma\left\{r_{i}\right\} \frac{\sin u}{a} \Delta u+\frac{\cos u}{a} \Delta r_{1}+\ldots+\frac{\cos u}{a} \Delta r_{N}, \\
& \frac{1}{\gamma} \Sigma\left\{y_{i}\right\}=0 . \Delta a+\frac{N}{\gamma} \Delta b+\Sigma\left\{r_{i}\right\} \frac{\cos u}{\gamma} \Delta u+\frac{\sin u}{\gamma} \Delta r_{1}+\ldots+\frac{\sin u}{\gamma} \Delta r_{s}, \\
& \Sigma\left\{r_{i}\left[-\frac{\sin u}{\alpha} \Delta x_{i}+\frac{\cos u}{\gamma} \Delta y_{i}\right]\right\} \\
& =-\Sigma\left\{r_{i}\right\} \frac{\sin u}{a} \Delta a+\Sigma\left\{r_{i}\right\} \frac{\cos u}{\gamma} \Delta b+\Sigma\left\{r_{i}\right\}^{2}\left[\frac{\sin ^{2} u}{a}+\frac{\cos ^{2} u}{\gamma}\right] \Delta u+r_{1}\left(\frac{1}{\gamma}-\frac{1}{a}\right) \cos u \sin u \Delta r_{1}+\ldots \\
& +r_{N}\left(\frac{1}{\gamma}-\frac{1}{a}\right) \cos u \sin u \Delta r_{N} \\
& \frac{\cos u}{a} \Delta x_{1}+\frac{\sin u}{\gamma} \Delta y_{1} \\
& =\frac{\cos u}{a} \Delta a+\frac{\sin u}{\gamma} \Delta b+r_{1}\left(\frac{1}{\gamma}-\frac{1}{a}\right) \cos u \sin u \Delta u+\left(\frac{\cos ^{2} u}{a}+\frac{\sin ^{2} u}{\gamma}\right) \Delta r_{1}+\ldots+0 \cdot \Delta r_{N}, \\
& \frac{\cos u}{a} \Delta x_{N^{*}}+\frac{\sin u}{\gamma} \Delta y_{x^{*}} \\
& =\frac{\cos u}{a} \Delta a+\frac{\sin u}{\gamma} \Delta b+r_{N}\left(\frac{1}{\gamma}-\frac{1}{a}\right) \cos u \sin u \Delta u+0 . \Delta r_{1}+\ldots+\left(\frac{\cos ^{2} u}{a}+\frac{\sin ^{2} u}{\gamma}\right) \Delta r_{N}
\end{aligned}
$$

Eliminating $r_{1}, r_{2} \ldots r_{N}$ from the first and the third of these equations by means of the last $N$ equations, we obtain and

$$
\Sigma\left\{\sin u \Delta x_{i}-\cos u \Delta y_{i}\right\}=N \sin u \Delta a-N \cos u \Delta b-\Sigma\left\{r_{i}\right\} \Delta u \ldots \ldots(120),
$$

$\Sigma\left\{r_{i}\left[\sin u \Delta x_{i}-\cos u \Delta y_{i}\right]\right\}=\Sigma\left\{r_{i}\right\} \sin u \Delta a-\Sigma\left\{r_{i}\right\} \cos u \Delta b-\Sigma\left\{r_{i}^{2}\right\} \Delta u \ldots(121)$. 
By eliminating the $r$ 's from the second of the normal equations we get an equation identical with (120), which shows that we have one more element than we can determine.

From (120) and (121) we are however able to find

$$
\begin{aligned}
& \text { ( } \sin u \Delta a-\cos u \Delta b \text { ) and } \Delta u \text {; we get } \\
& \sin u \Delta a-\cos u \Delta b=\frac{1}{N\left(m_{2}-m_{1}^{2}\right)} \Sigma\left\{\left(m_{2}-m_{1} r_{i}\right)\left(\sin u \Delta x_{i}-\cos u \Delta y_{i}\right)\right\} \\
& \text { and } \Delta u=\frac{1}{N\left(m_{2}-m_{1}^{2}\right)} \Sigma\left\{\left(m_{1}-r_{i}\right)\left(\sin u \Delta x_{i}-\cos u \Delta y_{i}\right)\right\} \text {, } \\
& \text { where } \quad m_{1}=\frac{1}{N} \Sigma\left\{r_{i}\right\} \text { and } m_{2}=\frac{1}{N} \Sigma\left(r_{i}^{2}\right) .
\end{aligned}
$$

For a point of the adjusted line corresponding to $r_{q}$ we find, according to (119),

$$
p_{q}=\sin u \Delta x_{q}-\cos u \Delta y_{q}=\sin u \Delta a-\cos u \Delta b-r_{p} \Delta u \text {. }
$$

The standard deviation of $p_{q}$ is seen to be the standard deviation of the position of the adjusted point $\left(x_{p}, y_{p}\right)$ in the direction at right angles to the line.

We find

and

$$
\begin{gathered}
p_{q}=\frac{1}{N\left(m_{2}-m_{1}^{2}\right)} \Sigma\left\{\left[m_{2}-m_{1} r_{i}-r_{p}\left(m_{1}-r_{i}\right)\right]\left(\sin u \Delta x_{i}-\cos u \Delta y_{i}\right)\right\} \\
\sigma_{p_{q}}^{2}=\frac{\sigma^{2}}{N}\left(\alpha \sin ^{2} u+\gamma \cos ^{2} u\right)\left\{1+\frac{\left(r_{p}-m_{1}\right)^{2}}{m_{\mathbf{2}}-m_{1}^{2}}\right\} .
\end{gathered}
$$

This standard deviation is quite analogous to that obtained for an adjusted ordinate when the abscissa is errorless and gives the same indications for the distribution of the observations.

For $\sigma_{u}$ we find

$$
\sigma_{u}^{2}=\frac{\sigma^{2}}{N} \frac{\left(\alpha \sin ^{2} u+\gamma \cos ^{2} u\right)}{\left(m_{2}-m_{1}^{2}\right)},
$$

again emphasising that the standard deviation of the $r$ 's ought to be a maximum to give the best determination of the line.

In conclusion I should like to express my thanks to Miss H. Gertrude Jones for the care she has devoted to the preparation of the diagrams in this paper. 